\title{
Automated Reasoning for Attributed Graph Properties
}

\author{
Sven Schneider ${ }^{1}$, Leen Lambers ${ }^{1}$, Fernando Orejas ${ }^{2}$ \\ Hasso Plattner Institut, University of Potsdam, Germany ${ }^{1}$ \\ Dpto de L.S.I., Universitat Politècnica de Catalunya, Barcelona, Spain ${ }^{2}$
}

The date of receipt and acceptance will be inserted by the editor

\begin{abstract}
Graphs are ubiquitous in Computer Science. Moreover, in various application fields graphs are equipped with attributes to express additional information such as names of entities or weights of relationships. Due to the pervasiveness of attributed graphs it is highly important to have the means to express properties on attributed graphs to strengthen modelling capabilities and to enable analysis.

Firstly, we introduce a new logic of attributed graph properties, where the graph and attribution part are neatly separated. The graph part is equivalent to first-order logic on graphs as introduced by Courcelle. It employs graph morphisms to allow the specification of complex graph patterns. The attribution part is added to this graph part by reverting to the symbolic approach to graph attribution, where attributes are represented symbolically by variables whose possible values are specified by a set of constraints making use of algebraic specifications.

Secondly, we extend our refutationally complete tableau based reasoning method as well as our symbolic model generation approach for graph properties to attributed graph properties. Due to the new logic mentioned above, neatly separating the graph and attribution part, and the categorical constructions employed only on a lower level we can leave the graph part of the algorithms seemingly unchanged. For the integration of the attribution part into the algorithms we use an oracle, allowing for flexible adoption of different available SMT solvers in the actual implementation.

Finally, our automated reasoning approach for attributed graph properties is implemented in the tool AUTOGRAPH integrating in particular the SMT solver Z3 for the attribute part of the properties. We motivate and illustrate our work with a particular application scenario on graph database query validation.
\end{abstract}

Key words: Attributed Graphs, Nested Graph Conditions, Model Generation, Tableau Method, Graph Queries

\section{Introduction}

Graphs are ubiquitous in Computer Science. Moreover, in various application fields graphs are equipped with attributes to express additional information such as names of entities or weights of relationships. Due to the pervasiveness of attributed graphs it is highly important to have the means to express properties on attributed graphs to strengthen modelling capabilities and to enable analysis. Properties on attributed graphs specify complex patterns on the graph structure and specify conditions on the attribute values of the graph. Examples of application areas include model-based engineering where properties of graphical models are expressed, the formal analysis and verification of systems where the states are modeled as graphs, the formal modeling and analysis of sets of semi-structured documents (especially if they are related by links), or of graph queries in the graph database domain.

As a first basic contribution we introduce a novel intuitive, dedicated logic for formulating attributed graph properties, where the graph and attribution part are neatly separated. The graph part uses graphs and graph morphisms as first-class citizens. In particular, we revert for this graph part to the logic of nested graph conditions as initially defined by Habel and Pennemann [21]. A similar approach was introduced by Rensink [43] first. The origins can be found in the notion of graph constraints [23], introduced in the area of graph transformation [44], in connection with the notion of (negative) application conditions [20,14], as a form to limit the applicability of graph transformation rules. These graph constraints originally had a very limited expressive power, while nested conditions have been shown $[21,39]$ to have the same expressive power as first-order logic (FOL) on graphs as introduced by Courcelle [9]. For integration of the attribution part with the graph part of the new logic for attributed graph properties we revert to the so-called symbolic approach to graph attribution [35], where the attributes are represented symbolically by variables whose possible values are specified by a set of constraints making use of algebraic specifications.

This is a post-peer-review, pre-copyedit version of an article published in International Journal on Software Tools for Technology Transfer. The final authenticated version is available online at: http://dx.doi.org/10.1007/s10009-018-0496-3. 
Apart from being able to express in an elegant and formal way attributed graph properties, we want to be able to automatically reason about these properties. A first question to be answered is if a given attributed graph property is satisfiable at all. We have addressed this question already in earlier work for graph properties without attributes [27]. In case an attributed graph property is satisfiable, a second question to be answered is which attributed graphs satisfy the property in particular. We have addressed this further question by a symbolic model generation approach for graph properties without attributes already in [46]. In particular, we identified that in most application scenarios it is desirable to be able to explore graphs satisfying the graph property or even to get a complete and compact overview of the graphs satisfying the graph property. More formally speaking, we designed an algorithm $\mathcal{A}$, which returns for a given graph property $p$ a finite set $\mathcal{S}$ of so-called symbolic models such that

- $\mathcal{S}$ jointly covers all graphs $G$ satisfying the graph property $p$ (completeness of $\mathcal{S}$ ),

- $\mathcal{S}$ does not cover any graph $G$ violating the graph property $p$ (soundness of $\mathcal{S}$ ),

- $\mathcal{S}$ contains no superfluous symbolic models not necessary for completeness (compactness of $\mathcal{S}$ ),

- $\mathcal{S}$ allows for each of its symbolic models the immediate extraction of a finite graph $G$, satisfying the graph property $p$ and being minimal (minimal representable $\mathcal{S}$ ),

- $\mathcal{S}$ allows for an enumeration of further finite graphs $G$ satisfying the graph property $p$ (explorable $\mathcal{S})$.

Such an algorithm is also desireable for attributed graphs.

The second main contribution of this paper is therefore twofold. We extend our refutationally complete tableau based reasoning method for graph properties [27] to the case with attributes. Moreover, we extend our symbolic model generation approach for graph properties [46] to the case with attributes. In addition, we show that it inherits all properties mentioned above of the algorithm for the non-attributed case also strengthening compactness. Moreover, we show that it comes up with an extra property for $\mathcal{S}$. In particular, we show that it does not generate symbolic models with overlapping covered attributed graphs (nonambiguity of $\mathcal{S}$ ). For showing that all other properties are inherited from the case without attributes, we exploit the fact that our new logic for attributed graph properties neatly separates the graph part from the attribution part and that our algorithm relies only on a lower level on categorical constructions specific to attributed graphs. Consequently, we can leave the graph part of previous algorithm seemingly unchanged. For the attribute part we assume and make use of an imaginary solver, allowing for flexible adoption of different available SMT solvers in the actual algorithm implementation. In fact, our refutation procedure and symbolic model generation algorithm for attributed graph properties are highly integrated: since the latter is designed to compute a complete overview of all possible models, it is at the same time able to refute a property if the overview turns out to be empty. Note that, in general, our symbolic model generation algorithm might not terminate because we sup- port FOL on graphs for the graph part already. It is designed however (also if non-terminating) to gradually deliver better underapproximations of the complete set of symbolic models by returning a stream of symbolic models.

Finally, we present the implementation of our algorithm in the tool AUTOGRAPH delegating the attribute part of the reasoning to the SMT solver Z3 [28]. We start the paper with presenting a concrete scenario, where our approach can be applied. In particular, we select the graph database domain $[3,53,2]$ and show that we can formally model and reason about the validity of graph database queries from a prominent case study in this domain on social network queries [51, version 0.3.0].

Compared to earlier work in [46] we extended the entire approach by allowing attribute constraints in graph properties, which strengthens its overall applicability. We have moreover added the property of nonambiguity and improved the notion of compactness. In particular, we have extended the application scenario started in [46], where we investigate the validity of graph database queries, by allowing for attribute constraints in the queries as illustrated by the examples in Figure 3, Figure 4, Figure 5, and Figure 6. This extension, which is based on earlier work on symbolic graphs making use of algebraic specifications from [35], requires the usage of SMT solvers such as Z3 at various steps in our model generation algorithm such as in Def. 19 (to attempt) to decide satisfiability of attribute constraints. Furthermore, exchanging the considered category from typed graphs to typed attributed graphs resulted in the need to inspect fundamental properties (see Appendix B) of the underlying category (i.e., the category of typed attributed graphs in our case) required for higher level constructions such as shift in Def. 17 according to their soundness proofs such as Lem. 1.

This paper is structured as follows: In section 2 we give an overview over related work. In section 3 we discuss graph databases as an application domain where graph queries are to be analyzed. In section 4 we recall the formalism of algebraic specifications required for attribute handling in a self contained way and introduce the category GRAPHSSTA of typed attributed graphs. In section 5 we introduce attributed graph properties over typed attributed graphs together with basic operations on them such as shift and conversion of graph properties into conjunctive normal form. In section 6 we adapt our tableau based reasoning procedure to typed attributed graphs, which has been initially developed in [27]. In section 7 we present the extension of our symbolic model generation algorithm [46] to attributed graph properties, and, in particular, show that it still fulfills the requirements listed before. In section 8 we describe the implementation of the algorithm in our tool AUTOGRAPH, focussing also on modifications required by extending our work to the handling of attributes and attribute constraints. In section 9 we take a closer look at our application scenario and apply our tool AUTOGRAPH to analyze the graph queries introduced before. We conclude the paper in section 10 together with an overview of future work. 


\section{Related Work}

Instead of using a dedicated logic for graph properties such as the one introduced in section 5 , graph properties may be defined in terms of some existing logic allowing the application of its associated reasoning methods. We structure the related work section in three parts. We start with describing approaches that follow the idea of using some existing logic and continue with approaches following the idea of working with a dedicated logic for graph properties. We conclude with a description of the integration of attribute constraints in all these approaches to automated reasoning on graph properties.

In particular, Courcelle presented in [9] a graph logic defined in terms of first-order (or monadic second-order) logic. In that approach, graphs are defined axiomatically using predicates node $(n)$, asserting that $n$ is a node and edge $\left(n_{1}, n_{2}\right)$ asserting that there is an edge from $n_{1}$ to $n_{2}$. In [18] such a translation-based approach for finding models of graphlike properties is followed. OCL properties are translated into relational logic and reasoning is then performed by KODKOD, which is a SAT-based constraint solver for relational logic. In a similar vein, in [4] reasoning for feature models is provided based on a translation into input for different general-purpose reasoners. Analogously, in [50] the ALLOY analyzer [50] is used to synthesize in this case large, wellformed and realistic models for domain-specific languages. Based on Alloy the Aluminum-tool [31] computes minimal models, which are smaller than a provided maximum (i.e., assuming the small world hypothesis from ALLOY), by minimizing initially non-minimal models computed by ALLOY. Moreover, ALUMINUM supports the interactive exploration of the model space (but is not compact as isomorphism is only approximated). However, ALUMINUM inherits some general problems from ALLOY: the use of a small world hypothesis, the required complex manual encoding, as well as the usage of general-purpose SMT solvers instead of domain specific solvers such as AUTOGRAPH limits the usability of Aluminum. Reasoning for domain specific modeling is addressed also in $[25,24]$ using the FORMULA approach taking care of dispatching the reasoning to the state-of-the-art SMT solver Z3 [28]. In [45] another translation-based approach is presented to reason with so-called partial models, which express uncertainty about the information in the model during model-based software development.

In principle, all the previously exemplarily presented approaches from the model-based engineering domain represent potential use cases for our automated reasoning approach for graph-like properties. We are in particular able to automatically refute graph properties as well as in case these properties are satisfiable to generate symbolic models being complete (in case of termination), sound, compact, minimally representable, and explorable in combination. We therefore believe that our approach has the potential to considerably enhance the type of analysis results, in comparison with the results obtained by using off-the-shelf SAT-solving technologies. Following this idea, in contrast to the translation-based approach it is possible, e.g, to formalize a graph-like prop- erty language such as OCL [42] by a dedicated logic for graph properties [21] and apply corresponding dedicated automated reasoning methods as developed in [37,38,34,27]. The advantage of such a graph-dedicated approach as followed in this paper is that graph axioms are natively encoded in the reasoning mechanisms of the underlying algorithms and tooling. Therefore, they can be built to be more efficient than generic-purpose methods, as demonstrated e.g. in [37, 38,39 ], where such an approach outperforms some standard provers working over encoded graph conditions. Moreover, the translation effort for each graph property language variant (such as e.g. OCL) into a formal logic already dedicated to the graph domain is much smaller than a translation into some more generic logic, which in particular makes translation errors less probable. Another approach following this idea is presented in [49] where uncertainty about a graphbased model, which possibly occurs in partial models, may be resolved by graph transformation steps. As most directly related work [37,39] present a satisfiability solving algorithm for graph properties [21]. This solver attempts to find one finite model (if possible), but does not generate a compact and gradually complete finite set of symbolic models allowing to inspect all possible finite models including a finite set of minimal ones. In contrast to [37,39] our symbolic model generation algorithm is interleaved directly with a refutationally complete tableau based reasoning method [27], inspired by rules of a proof system presented previously in [38], but in that work the proof rules were not shown to be refutationally complete.

When it comes to integrating attribute constraints in all the previous approaches mentioned above we can observe that most translation-based approaches usually come with attribute support for the properties and the corresponding reasoning. This is presumably because they can rely on the underlying solvers for coping with attribute constraints. All dedicated automated reasoning approaches up until now do not support attributes. However, there exists one recent work [11] integrating attribute support into static analysis techniques for graph transformation. As in our approach it is based on formalizing graph attribution using symbolic graphs as introduced in $[35,36]$. In particular, the tool SYGRAV and a framework for the analysis of symbolic graph transformation systems also makes use of the Z3 solver to handle attribute constraints. SYGRAV allows the usage of nested negative application conditions, which are used to restrict rule applications in symbolic graph transformation systems. This approach however is not concerned with automated reasoning for attributed graph properties.

\section{Application Scenario}

In this section we focus on an application scenario from the graph database domain in which our automated reasoning approach for attributed graph properties can be applied. We revert to a prominent case study in this domain concerned with social network queries [51, version 0.3 .0$, p. 25]. It was devel- 


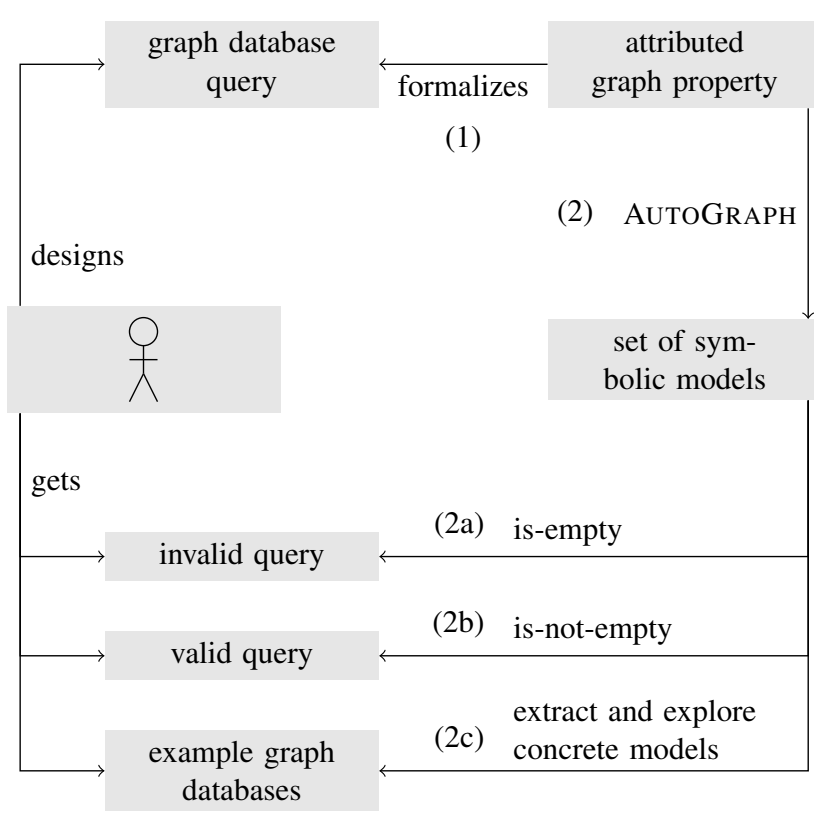

Figure 1: Application scenario (nonemptiness problem)

oped by the Linked Data Benchmark Council (LDBC) as a benchmark for following up the progress in graph data management technologies.

Analogous to the relational database domain [48] queries can be formalized and validated by subjecting them to static analysis. As argued also in the relational domain, querying a database should not depend on how and where the data is stored. For relational databases the relational model [8] has therefore been designed as an underlying theory for modeling databases and their queries with the mathematical notion of a relation. A relational database consists of one (or more) relations of arity prescribed by the given database schema. A relational query is basically a mapping of a given database to a relation of fixed arity. Following this idea (cf. [6] and [26]) graphs and graph properties have been used to model graph databases and their queries. In this graph model a graph $G$ (possibly typed over a given type graph $T G$ ) models a graph database instance. A graph query $q$ for a graph database $G$ maps $G$ to one (or more) patterns (or subgraphs) of a specific form in $G$.

The first step in our application scenario (cf. (1) in Figure 1) is the formalization of graph queries as attributed graph properties: we described the first four "complex read queries" from the LDBC Social Network Benchmark case study [51, version 0.3 .0$, p. 25] into the typed attributed graph properties presented in Figure 3, Figure 4, Figure 5, and Figure 6. Note, arguments of the graph queries are translated into variables $\arg _{i}$ in their graph property counterparts. Technically, graph properties allow for the combination (by propositional operators such as conjunction and negation) of statements of existence of (sub)graphs in a given host graph also allowing more complex statements using nesting. In addition to graph conditions such as in $[21,46]$, we also allow for the usage of attributes and attribute constraints.
The second step in our application scenario (cf. (2) in Figure 1) is the application of our automated reasoning approach for graph query analysis. As described also for the relational domain [48] many query optimization tasks rely on the following three types of questions to be answered (cf. (2a)(2c) in Figure 1): Can a query $q$ ever deliver a nonempty result (nonemptiness problem)? Does query $q_{1}$ always deliver the same result as query $q_{2}$ (equivalence problem)? Is the result of query $q_{1}$ always contained in the result of query $q_{2}$ (containment problem)? In particular, the first question that can be answered automatically by our approach for a given graph database query is if there exists a graph database for which the query result is non-empty as illustrated in Figure 1. In particular, our refutationally complete reasoning method for attributed graph properties implemented in our tool $\mathrm{Au}$ toGraph returns an empty set of symbolic models iff there exists no model for the given attributed graph property. Then we know that the graph database query is invalid. On the other hand, our symbolic model generation algorithm returns a non-empty set of symbolic models iff there exist finite models for the attributed graph property. In this case, we know that the original query is valid. In addition however we can explore minimal example models for the property that can be extracted immediately from each symbolic model. For our application scenario this means that we get example graph databases for our query that deliver non-empty results. In fact, the symbolic models generated describe all different minimal graph databases that can serve as witnesses for the validity of a query because our symbolic model generation algorithm delivers a finite, complete, minimal representable, nonambiguous and compact overview of all graph databases delivering a non-empty result. Finally, a flexible exploration starting from any such minimal graph database to bigger ones that are still witnesses for the validity will be feasible by checking suitable candidates (constructed as supergraphs of the minimal models using atomic graph modifications) for being still witnesses for the validity of the given query.

In the graph database domain, compared to the relational domain, it is not common to have a database schema but often a conceptual model [10] is present. Such a conceptual model is given by the UML-Class Diagram depicted (adapted with minor corrections and completions from the LDBC Social Network Benchmark) in Figure 2 for our case study. We can integrate such conceptual models describing the structure of all graph databases to be queried into our graph model and analysis approach by flattening the inheritance structure of the UML-Class Diagram to obtain a type graph, by modelling the multiplicity constraints as special attributed graph properties (cf. to Figure 10c and Figure 10d for examples), and by forbidding parallel edges of the same type by graph properties as in Figure 10b.

Finally, with our application scenario we can also answer, analogous to the nonemptiness problem, further questions when given multiple graph queries at once. Firstly, the nonemptiness problem can be answered for a set of queries by using a conjunction of attributed graph properties instead of a single one. Such a set of queries is then jointly valid if they 
are able to deliver non-empty answer sets on a common graph. Secondly, we could answer the abovementioned equivalence or containment problem for two given queries as a basis for query optimization (analogous to query optimization in the relational database domain $[1,48]$ ). For two graph queries $q_{1}$ and $q_{2}$ we can state their equivalence $q_{1} \equiv q_{2}$ or containment $q_{1} \subseteq q_{2}$ using a logical equivalence or implication in a single nested graph condition.

The analysis questions introduced in our application scenario here are answered for the example graph queries of our case study mentioned above in our evaluation in section 9 by using our automated reasoning approach for attributed graph properties implemented in the tool AUTOGRAPH.

\section{Preliminaries}

In this section we provide, in a self-contained way, fundamentals, which are used in the remainder of this paper. Firstly, in subsection 4.1 we introduce algebraic specifications along the lines of [16]. Secondly, in subsection 4.2 we introduce symbolic typed attributed graphs based on algebraic specifications from $[35,36]$.

\subsection{Algebraic Specifications}

We introduce in our notation the well-known algebraic specifications, which are used in this paper for the handling of attribute values in subsection 4.2. Algebraic specifications can be used to describe data and functional programs. Furthermore, since SMT solvers such as Z3 support the formalism of algebraic specifications, we are able to employ them for our purposes in subsequent sections.

A signature consists of sorts $S$ and symbols for operations $O$. The elements of $O$ are equipped with a list of input sorts and a unique output sort. Elements of $O$ with an empty list of input sof sorts are called constants.

Definition 1 (Signature). $\Sigma^{p}=\left(S, O\right.$, type $\left._{O}: O \rightarrow S^{+}\right)$ is a signature if $S$ and $O$ are finite.

To allow for the symbolic handling of attribute values (using terms and equations later on) we equip signatures with sorted variables distinguishable from the operations of the signature.

Definition 2 (Signature with Variables). Given a signature $\Sigma^{p}$ (as in Def. 1). $\Sigma=\left(\Sigma^{p}, X\right.$, type $\left._{X}: X \rightarrow S\right)$ is a signature with variables if $X \cap O=\emptyset$.

Example 1 (Signature with Variables). We employ the wellestablished notation for signatures with variables as for the following signature, which captures boolean expressions (in Def. 30 we provide a signature only including the built-in operations support by AUTOGRAPH via Z3).

$$
\begin{aligned}
& \text { sorts: bool } \\
& \text { opns: true : } \rightarrow \text { bool } \\
& \text { false }: \rightarrow \text { bool } \\
& \text { not : bool } \rightarrow \text { bool } \\
& \text { and }: \text { bool bool } \rightarrow \text { bool } \\
& \text { vars: } b_{1}, b_{2}: \text { bool }
\end{aligned}
$$

Amongst other usages (explained later on), we specify attribute values in subsection 4.2 based on (the recursively defined) terms over a given signature. The terms are well-typed in the sense that they respect the sorts declared for variables, constants, and operations. Note, we define the terms for subsets of the variables $X$ given in the signature with variables.

Definition 3 (Terms). Given a signature with variables $\Sigma$ (as in Def. 2), $s \in S$, and $X^{\prime} \subseteq X$. We define $T_{\Sigma, s}\left(X^{\prime}\right)$ to be the smallest set s.t.

$$
\begin{aligned}
& -x \in T_{\Sigma, s}\left(X^{\prime}\right) \text { if type }{ }_{X}(x)=s \text { and } x \in X^{\prime}, \\
& -c \in T_{\Sigma, s}\left(X^{\prime}\right) \text { if } c \in O \text { and type } O(c)=s \text {, and } \\
& -f\left(t_{1}, \ldots, t_{n+1}\right) \in T_{\Sigma, s}\left(X^{\prime}\right) \text { if } f \in O, \text { type }_{O}(f)=s_{1} \ldots \\
& \left.\quad s_{n+1} \cdot s, \text { and } t_{i} \in T_{\Sigma, s_{i}}\left(X^{\prime}\right) \text { (for each } 1 \leq i \leq n+1\right) .
\end{aligned}
$$

Also, we define

$$
\begin{aligned}
& -\operatorname{sort}_{\Sigma}(t)=s \text { whenever } t \in T_{\Sigma, s}(X), \\
& -T_{\Sigma, \star}\left(X^{\prime}\right)=\bigcup_{s \in S} T_{\Sigma, s}\left(X^{\prime}\right), \text { and } \\
& -T_{\Sigma, \star}=T_{\Sigma, \star}(\emptyset) .
\end{aligned}
$$

The last two items from the previous definition are to be understood as follows. On the one hand, terms of arbitrary type without variables (such as and(true, false) of sort bool when using the signature in Example 1) are collected in the set $T_{\Sigma, \star}$ and represent values. On the other hand, terms containing variables are used to describe sets of values in a symbolic way, e.g., the statements $x>4$ and endsWith( $s$, "suffix") where $x$ and $s$ are variables can be expressed by using signatures with variables comprising the used integer and string operations and variables, respectively.

Variable substitutions are required for manipulation of terms such as in instantiations, simplifications, and equivalence proofs. A variable substitution determines for each variable $x$ (possibly occurring in a term $t$ ) a replacement term (of equal type) to be inserted in $t$ for each occurrence of the variable $x$

Definition 4 (Variable Substitution). Given the two signatures with variables $\Sigma_{1}=\left(\Sigma^{p}, X_{1}\right.$, type $\left.x_{X_{1}}\right)$ and $\Sigma_{2}=\left(\Sigma^{p}\right.$, $X_{2}$, type $_{X_{2}}$ ) with common underlying signature $\Sigma^{p}$ (as in Def. 2). Each function $\sigma: A \rightarrow B$ where $A \subseteq X_{1}, X_{1}-$ $X_{2} \subseteq A$ (that is, $\sigma$ replaces at least the variables not known by $\left.\Sigma_{2}\right)$, and $B \subseteq T_{\Sigma_{2}, \star}\left(X_{2}\right)$ is a variable substitution, written $\sigma \in \mathcal{V}_{\Sigma_{1}, \Sigma_{2}}$, if $\operatorname{sort}_{\Sigma_{2}}(\sigma(x))=\operatorname{sort}_{\Sigma_{1}}(x)$ for each $x \in$ $A$. Furthermore, the variable substitution $\sigma$ is implicitly extended to a function of type $T_{\Sigma_{1}, \star}\left(X_{1}\right) \rightarrow T_{\Sigma_{2}, \star}\left(X_{2}\right)$, which recursively replaces all occurrences of a variable $x \in A$ in a given term by the replacement $\sigma(x) \in B$. 


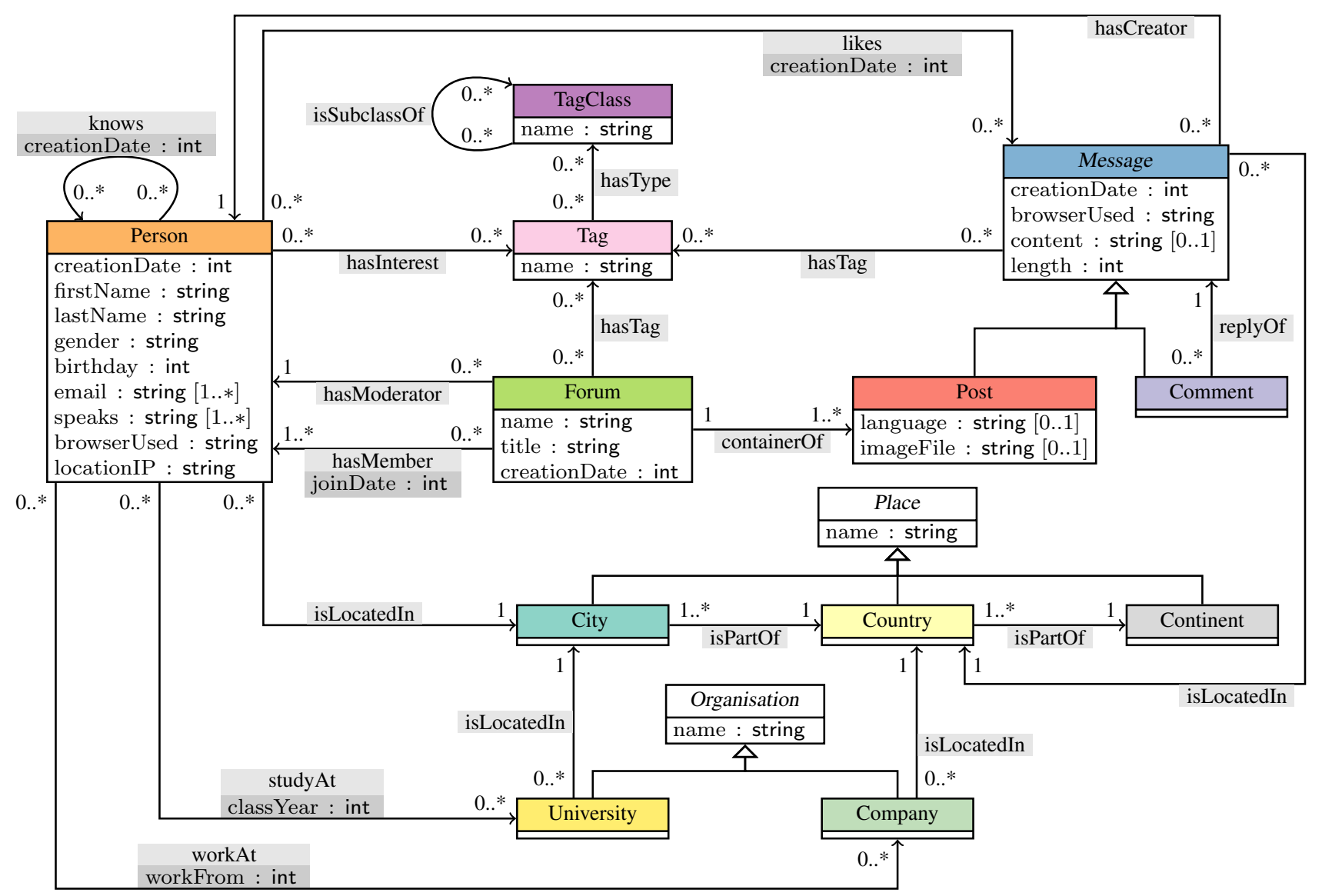

Figure 2: Adapted UML-Class Diagram of the LDBC Social Network Benchmark [51, version 0.3.0, p. 15]

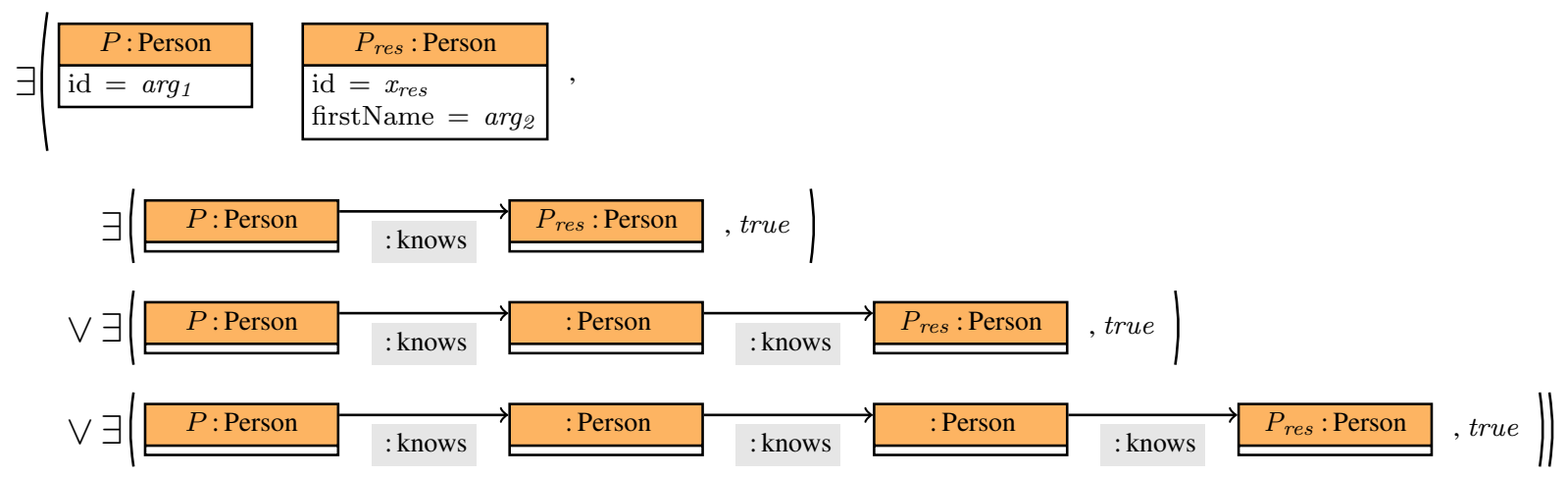

Figure 3: Graph property $p^{3}$ modelling query 1 of the LDBC Social Network Benchmark [51, version 0.3.0, p. 25] searching for persons $P_{\text {res }}$ (with a given firstName-value of $\arg _{2}$ ) that are reachable by a path of knows-edges of length 1-3 from a person $P$ given by the id $\arg _{1}$.

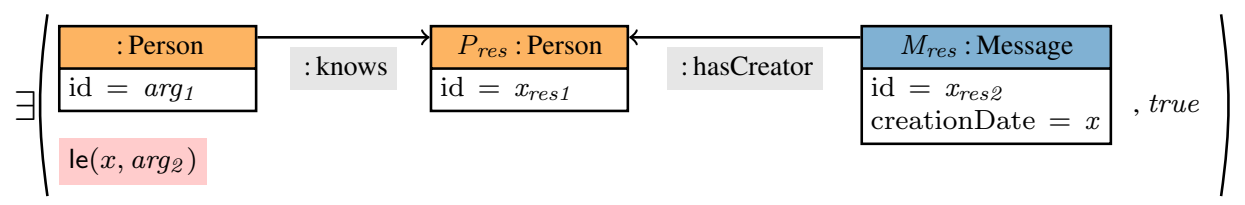

Figure 4: Graph property $p^{4}$ modelling query 2 of the LDBC Social Network Benchmark [51, version 0.3.0, p. 25] searching for persons $P_{\text {res }}$ (known by a person given by the id $\arg _{1}$ ) and for messages $M_{\text {res }}$ created by $P_{\text {res }}$ before a given time $\arg _{2}$. 

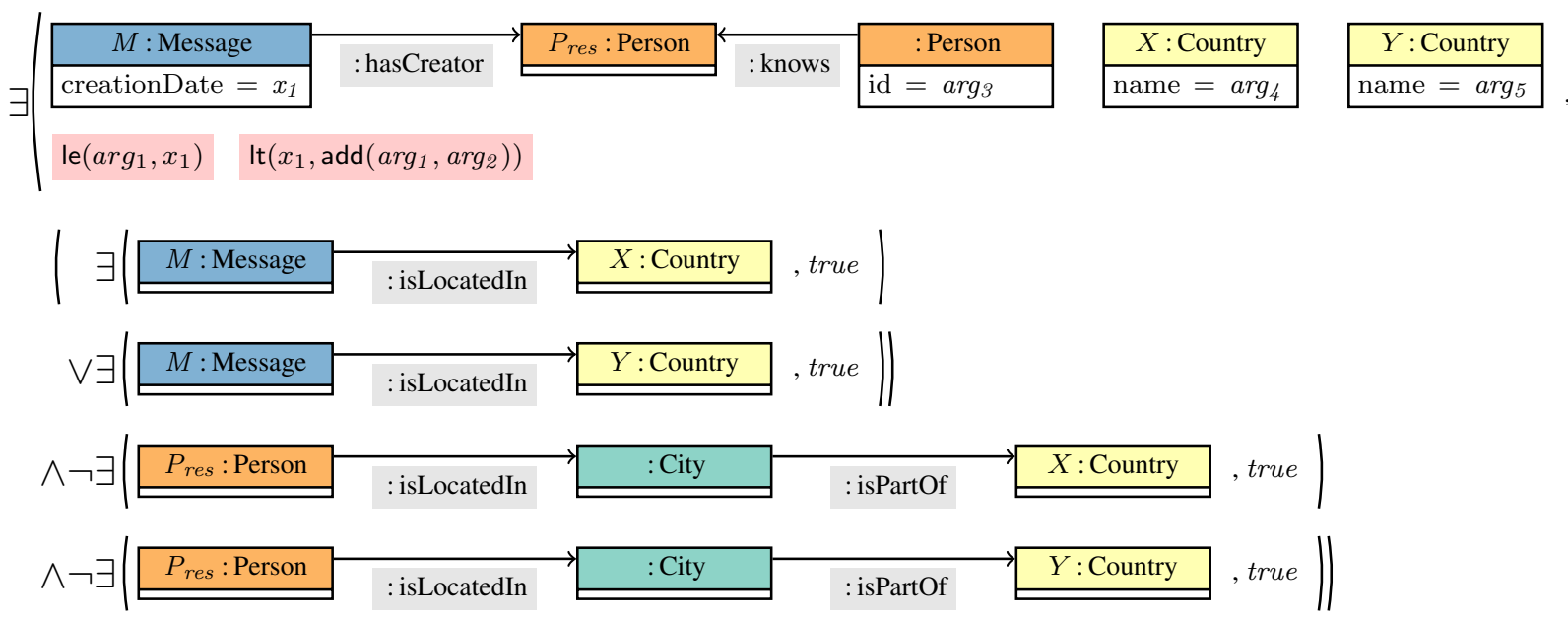

Figure 5: Graph property $p^{5}$ modelling query 3 of the LDBC Social Network Benchmark [51, version 0.3.0, p. 25] searching for persons $P_{\text {res }}$ (known by a person given by the id $\arg _{3}$ ) that created at least one message $M$ in the time interval $\left[\arg _{1}, \arg _{1}+\right.$ $\arg _{2}$ ) from country $X$ or $Y$ (with names given by $\arg _{3}$ and $\arg _{4}$, respectively) without being located in $X$ or $Y$.

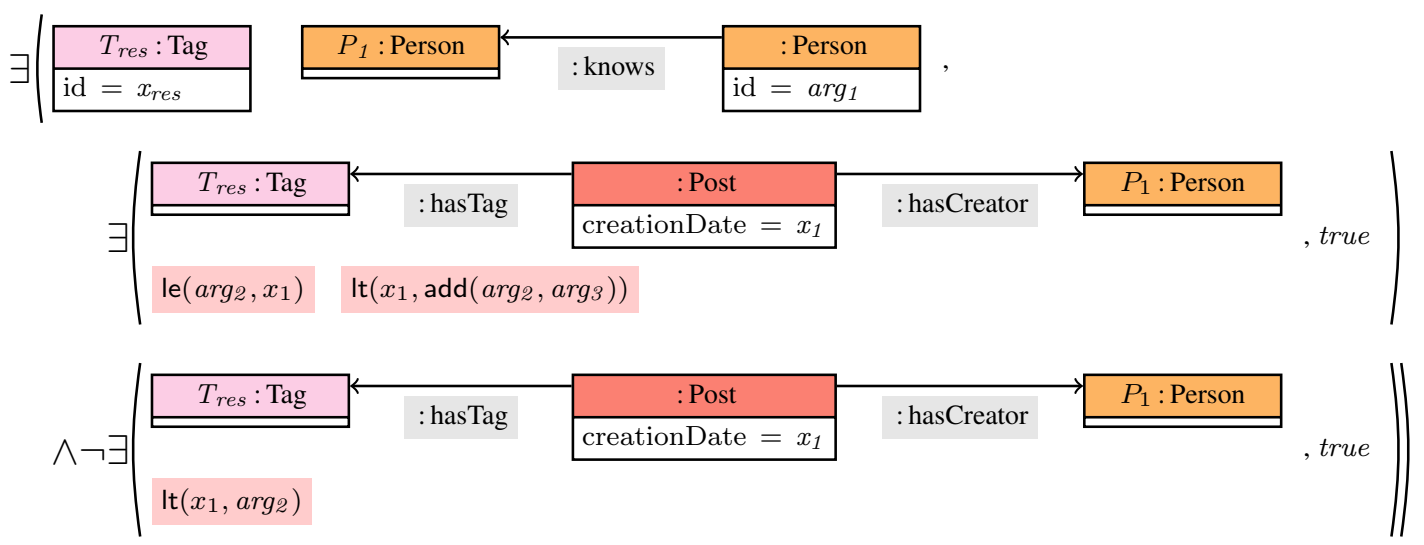

Figure 6: Graph property $p^{6}$ modelling query 4 of the LDBC Social Network Benchmark [51, version 0.3.0, p. 25] searching for tags $T_{r e s}$ that are attached to posts created (in the time interval $\left[\arg _{2}, \arg _{2}+\arg _{3}\right)$ ) by a person known by a person given by the id $\arg _{2}$ such that, in addition, there are no posts created by such persons before that time interval.

An algebraic specification extends a signature with variables by a finite set of term rewrite rules, which are called equations. The equations are of the form $(\ell, r)$ where $\ell$ and $r$ are terms of equal sort, possibly making use of variables, and are usally written $\ell=r$. The equations are used to resolve on the one hand semantic confusion between terms to be considered equals (e.g., zero $=\operatorname{minus}($ zero $)$ ). On the other hand, equations determine the semantics of an operation contained in the signature as in functional programming languages (consider the equations from Example 2). For many relevant theories these equations are applied as in functional programming from left to right to simplify terms; in particular terms without variables.

Definition 5 (Algebraic Specification). Given a signature with variables $\Sigma$ (as in Def. 2$) . S P=(\Sigma, E Q)$ is an $(a l$ gebraic) specification if

$$
-E Q \subseteq T_{\Sigma, \star}(X)^{2}
$$

- $E Q$ is finite, and
- $\operatorname{sort}(\ell)=\operatorname{sort}(r)$ for each $(\ell, r) \in E Q$.

The equations of the following algebraic specification can be used to rewrite terms containing the operations and and not into equivalent terms (defined below) without these operations (i.e., into the terms true or false).

Example 2 (Algebraic Specification). Equations of type bool that could be added to the signature from Example 1 leading to a reasonable algebraic specification are:

$$
\begin{aligned}
& \text { eqns: } \text { and }(\text { true }, \text { true })=\text { true } \\
& \text { and }\left(\text { false }, b_{1}\right)=\text { false } \\
& \text { and }\left(b_{1}, \text { false }\right)=\text { false } \\
& \operatorname{not}(\text { false })=\text { true } \\
& \operatorname{not}(\text { true })=\text { false }
\end{aligned}
$$


The equations of a specification already determine certain terms to be equivalent but this basic equivalence is insufficient and is therefore extended to a congruence $\cong$ w.r.t. the operators from the signature by allowing application of equations to subterms. As a base case, a term $t_{1}$ can be rewritten into an equivalent term $t_{2}$ by use of an equation $(\ell, r)$ of the specification at hand by replacing (using a variable substitution $\sigma$ fixing the variables occurring in the equation) $t_{1}=\sigma(\ell)$ by $t_{2}=\sigma(r)$.

Definition 6 (Equivalence of Terms). Given a specification $S P$ (as in Def. 5). We define $\cong_{1} \subseteq T_{\Sigma, \star}(X)^{2}$ to be the least equivalence s.t. $t_{1} \cong_{1} t_{2}$ whenever there are $(\ell, r) \in E Q$ and $\sigma \in \mathcal{V}_{\Sigma, \Sigma}$ s.t. $t_{1}=\sigma(\ell)$ and $t_{2}=\sigma(r)$.

The congruence $\cong$ mentioned above is obtained by allowing term rewritings based on $\cong_{1}$ on any level.

Definition 7 (Congruence of Terms). Given a specification $S P$ (as in Def. 5). We define $\cong \subseteq T_{\Sigma, \star}(X)^{2}$ to be the least equivalence s.t. $t_{1} \cong t_{2}$ if

$-t_{1} \cong_{1} t_{2}$ or

- there is some $f \in O$ with type $O(f)=s_{1} \cdots s_{n+1} \cdot s$, $t_{i}^{j} \in T_{\Sigma, s_{i}}(X)$ (for each $1 \leq i \leq n+1$ and $1 \leq$

$j \leq 2$ ) with $t_{i}^{1} \cong t_{i}^{2}$ (for each $1 \leq i \leq n+1$ ) and

$t_{j}=f\left(t_{1}^{j}, \ldots, t_{n+1}^{j}\right)($ for each $1 \leq j \leq 2)$.

Example 3 (Congruence of Terms). Using Equation 1 from Example 2 we can simplify the term and(true, false) to false by term rewriting (using either ${ }_{1}$ or $\cong$ ).

Subsequently we implicitly assume that algebraic specifications include a propositional fragment based on the sort bool as well as the propositional constants true (required for satisfiability in Def. 8) and false (required for type graphs as discussed in the paragraph preceding Def. 12).

We call terms of type bool (attribute) constraints and use them in attributed graphs to specify/restrict variable values. For these constraints we define the satisfaction as follows.

Definition 8 (Satisfaction of Constraints). For a specification $S P$ (as in Def. 5), a single constraint $\phi \in T_{\Sigma \text {,bool }}(X)$ (as in Def. 3), a set $\Phi \subseteq T_{\Sigma \text {,bool }}(X)$ of constraints, and a variable substitution $\sigma \in \mathcal{V}_{\Sigma, \Sigma}$ (as in Def. 4) we define that

- $\sigma$ satisfies $\phi$, written $\sigma \models \phi$, if $\sigma(\phi) \cong$ true,

- $\phi$ is satisfiable by $S P$ if $\exists \sigma \in \mathcal{V}_{\Sigma, \Sigma} \cdot \sigma \models \phi$,

- $\sigma$ satisfies $\Phi$, written $\sigma \models \Phi$, if $\forall \phi \in \Phi . \sigma \models \phi$,

- SP satisfies $\phi$, written $S P \models \phi$, if $\forall \sigma \in \mathcal{V}_{\Sigma, \Sigma} \cdot \sigma \models \phi$,

- $\Phi$ is satisfiable by $S P$ if $\exists \sigma \in \mathcal{V}_{\Sigma, \Sigma} . \forall \phi \in \Phi . \sigma \models \phi$, and

- SP satisfies $\Phi$, written $S P \models \Phi$, if $\forall \phi \in \Phi$. $S P \models \phi$.

SMT solvers such as Z3 are typically shipped with built-in datatypes, operations, and equations on, e.g., booleans, integers, and strings. Thus, in practice, users of such solvers and, hence, also users of higher level tools such as AUTOGRAPH do not need to construct custom algebraic specifications and custom equations when using such built-in datatypes only.
Note, users of our tool AUTOGRAPH may extend the basic algebraic specification to introduce further sorts and operations (including equations) for use in constraints in attributed graphs. However, while SMT solvers are often sufficient for the few built-in fragments mentioned before, satisfaction and satisfiability of constraints can not be decided for arbitrary specifications with more complex equations. Constraints where the employed SMT solver is not capable of deciding satisfiability pose problems to our model generation procedure: this handling of this problem is explicitly addressed in section 8 .

\subsection{Symbolic Typed Attributed Graphs}

Symbolic typed attributed graphs, as introduced in [35, Definition 1] together with a framework of graph transformation on these graphs, may include vertex attributes and edge attributes similarly to EGRAPHS [13]. That is, any number of vertex attributes (edge attributes) are mapped on the one hand to a vertex (an edge), called source, and, on the other hand, to an assigned element, called target. However, in EGRAPHS the target of an attribute is an element of the employed data algebra wheras in symbolic typed attributed graphs the target is a variable of the algebraic specification. Moreover, in symbolic typed attributed graphs a set of constraints is used to describe the values that such a variable can take. Note, the set of constraints may be satisfiable by more than one variable substitution. A set of constraints is more expressive than a single constaint because only a finite set of constraints can be translated in general into a single constraint using finite conjunction in the obvious way.

We introduce the category of symbolic typed attributed graphs step-wise starting with plain graphs.

Definition 9 (Plain Graph). Plain graphs $G^{p}$ are tuples of the form $(V, E, \operatorname{src}: E \rightarrow V, \operatorname{trg}: E \rightarrow V)$.

Note, to simplify notation we facilitate the usual notation for selectors for tuples such as for plain graphs. E.g., we denote the vertices of a plain graph $G_{i}^{p}$ by $V_{G_{i}^{p}}$ or simply by $V_{i}$.

In the next step plain graphs are extended by an algebraic specification, an attribution consisting of attribute variables, attribute vertices, and attribute edges together with the source and target mappings similarly available in EGRAPHS, and constraints restricting the possible values of the attribute variables.

Definition 10 (Symbolic Attributed Graph). Given a plain graph $G^{p}$ (as in Def. 9), a specification $S P$ (as in Def. 5) with finite set $X$, and an attribution $A=\left(A X\right.$, type $_{A X}: A X \rightarrow$ $S, A V, \operatorname{src}_{A V}: A V \rightarrow V, \operatorname{trg}_{A V}: A V \rightarrow A X, A E, \operatorname{src}_{A E}:$ $\left.A E \rightarrow E, \operatorname{trg}_{A E}: A E \rightarrow A X\right)$. Then $G^{s a}=\left(G^{p}, S P, A, \Phi\right)$ is a symbolic attributed graph if there is a signature $\Sigma_{A}$ s.t.

$$
\begin{aligned}
& -X \cap A X=\emptyset, \\
& -\Sigma_{A}=\left(\Sigma^{p}, X \cup A X, \text { type }_{X} \cup \text { type }_{A X}\right) \text {, and } \\
& -\Phi \subseteq T_{\Sigma_{A}, \text { bool }}(A X)
\end{aligned}
$$

See Figure 7 for an example of symbolic attributed graph and the simplified notation. Note, the empty graph, written $\emptyset$, is 


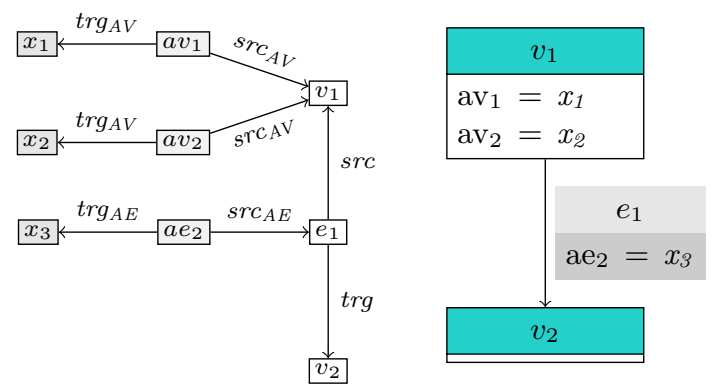

and $\left(x_{1}, \operatorname{not}\left(\operatorname{and}\left(x_{2}, x_{3}\right)\right)\right)$

Figure 7: A symbolic attributed graph $G^{7}$ in two notations (left/right). The used specification from Example 2 is not depicted. The single constraint $\Phi$ is depicted separately from the graph structure at the bottom. Note, the constraint can be satisfied by three different variable substitutions.

assumed to have either the constraint set $\Phi=\emptyset$ or $\Phi=\{$ true $\}$ when this fact is to be expressed more explicitly as in Figure 12 .

The subsequently introduced notion of a morphisms between symbolic attributed graphs is extended with compatibility w.r.t. typing in Def. 14. As for now the morphisms map vertices, edges, and attributes in a way compatible with the various source and target operations (see Figure 9). Also note, attribute variable mappings must not restrict satisfying variable substitutions, i.e., a variable substitution $\sigma$ satisfying the constraint of the target symbolic attributed graph $G_{2}^{s a}$ must already have satisfied the constraint of the source symbolic attributed graph $G_{1}^{s a}$ after application of the symbolic attributed graph morphism. For example, consider the symbolic (typed) attributed graph morphism $m_{4}$ from Figure 8 , where each variable substitution satisfying the constraint of $G_{4}$ also satisfies the constraint of $G_{2}$ (in this example no variable renaming is performed by $m_{4}$ as it maps the attribution variable $x$ from $G_{2}$ to the attribution variable $x$ from $\left.G_{4}\right)$.

Definition 11 (Symbolic Attributed Graph Morphism). If $G_{1}^{s a}$ and $G_{2}^{s a}$ are symbolic attributed graphs (as in Def. 10) with specification $S P=(\Sigma, E Q)$, then $f=\left(f_{V}: V_{1} \rightarrow V_{2}\right.$, $f_{E}: E_{1} \rightarrow E_{2}, f_{A X}: A X_{1} \rightarrow A X_{2}, f_{A V}: A V_{1} \rightarrow A V_{2}$, $\left.f_{A E}: A E_{1} \rightarrow A E_{2}\right)$ is a symbolic attributed graph morphism of type $G_{1}^{s a} \rightarrow G_{2}^{s a}$, written $f: G_{1}^{s a} \rightarrow G_{2}^{s a}$, if (see Figure 9)

$$
\begin{aligned}
& -\operatorname{src}_{2} \circ f_{E}=f_{V} \circ \operatorname{src}_{1}, \\
& -\operatorname{trg}_{2} \circ f_{E}=f_{V} \circ \operatorname{trg}_{1}, \\
& -\operatorname{src}_{A V_{2}} \circ f_{A V}=f_{V} \circ \operatorname{src}_{A V_{1}}, \\
& -\operatorname{trg}_{A V_{2}} \circ f_{A V}=f_{A X} \circ \operatorname{trg}_{A V_{1}}, \\
& -\operatorname{src}_{A E_{2}} \circ f_{A E}=f_{E} \circ \operatorname{src}_{A E_{1}}, \\
& -\operatorname{trg}_{A E_{2}} \circ f_{A E}=f_{A X} \circ \operatorname{trg}_{A E_{1}}, \\
& -\operatorname{type}_{A X_{2}} \circ f_{A X}=\operatorname{type}_{A X_{1}} \text {, and }
\end{aligned}
$$

$$
\begin{aligned}
& \text { - for all } \sigma \in \mathcal{V}_{\Sigma_{A_{2}}, \Sigma_{A_{2}}}:^{1} \\
& \quad \sigma \models \Phi_{2} \text { implies } \sigma \models f_{A X}\left(\Phi_{1}\right) .
\end{aligned}
$$

Moreover, the composition $f_{2} \circ f_{1}: G_{1}^{s a} \rightarrow G_{3}^{s a}$ of symbolic attributed graph morphisms $f_{1}: G_{1}^{s a} \rightarrow G_{2}^{s a}$ and $f_{2}: G_{2}^{s a} \rightarrow$ $G_{3}^{s a}$ is defined componentwise.

A symbolic attributed graph $G^{s a}$ is extended to a symbolic typed attributed graph $G$ (or graph for short) when a type graph $T G$ is available such that a typed attributed graph morphism from $G^{s a}$ to $T G$ can be determined, which, by definition, has to satisfy the compatibilities discussed before. In practice we often use the single constraint false for type graphs to allow arbitrary values in symbolic attributed graphs to be typed.

Definition 12 ((Symbolic Typed Attributed) Graph). For a given symbolic attributed graph morphism $\tau: G^{s a} \rightarrow T G$ (as in Def. 11) we define $G=\left(G^{s a}, T G, \tau: G^{s a} \rightarrow T G\right)$ to be a (symbolic typed attributed) graph.

We introduce grounded graphs as those symbolic typed attributed graphs where the set of attribute constraints $\Phi$ can be satisfied by a unique variable substitution, whereas for typed attributed graphs also infinite and empty sets of such variable substitutions are allowed.

Definition 13 (Grounded Graph). A graph is a grounded graph, if its set of attribute constraints $\Phi$ is satisfiable by a unique variable substitution. For a graph $G_{s}$ we denote all grounded graphs $G_{g}$ obtainable from $G_{s}$ by only adding further attribute constraints to $G_{s}$ by $\operatorname{grounded}\left(G_{s}\right)$.

Subsequently, we assume a fixed symbolic attributed graph $T G$ used as a type graph and lift symbolic attributed graph morphisms to symbolic typed attributed graphs by requiring that the two symbolic attributed graph morphisms used for typing are compatible in the sense of the commutation of the triangle in the following definition.

Definition 14 ((Symbolic Typed Attributed) Graph Morphism). Given two (symbolic typed attributed) graphs $G_{1}=$ $\left(G_{1}^{s a}, T G, \tau_{1}\right)$ and $G_{2}=\left(G_{2}^{s a}, T G, \tau_{2}\right)$ (as in Def. 12) over a common type graph $T G$ and a symbolic attributed graph morphism $f: G_{1}^{s a} \rightarrow G_{2}^{s a}$ (as in Def. 11). We define $f$ to be a (symbolic typed attributed graph) morphism and also to be of type $G_{1} \rightarrow G_{2}$ if $\tau_{2} \circ f=\tau_{1}$.

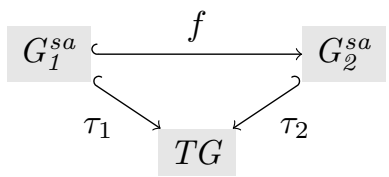

We are binding the definitions of graphs and morphisms from before into the single notion of a category.

\footnotetext{
1 Firstly, the algebraic specification used is given by $\left(\Sigma_{A_{2}}, E Q\right)$ where $\Sigma_{A_{2}}$ is the signature with variables obtained for the attribution $A_{2}$ of $G_{2}^{s a}$ and the signature $\Sigma$ from $S P$ as in Def. 10. Secondly, $f_{A X}$ : $A X_{1} \rightarrow A X_{2}$ is a member of $\mathcal{V}_{\Sigma_{A_{1}}, \Sigma_{A_{2}}}$ according to Def. 4.
} 


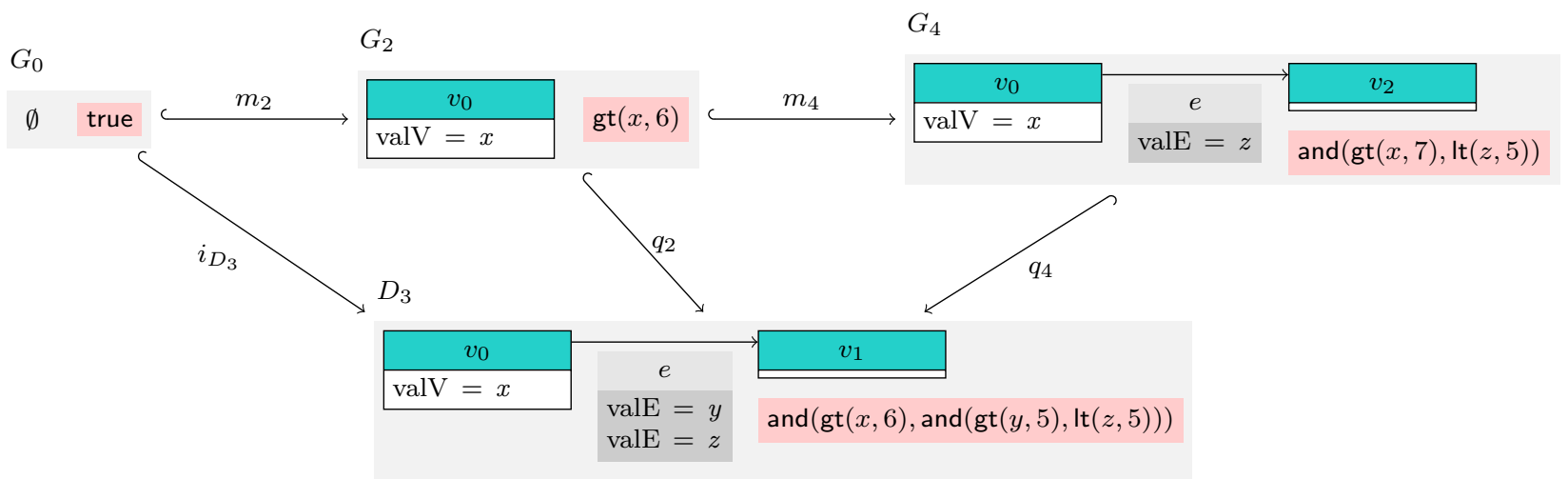

Figure 8: Five symbolic (typed) attributed graph morphisms. The type graph is given in Figure 12a. Also, $D_{3}$ satisfies the graph property $\exists\left(m_{2}: G_{0} \hookrightarrow G_{2}, \exists\left(m_{4}: G_{2} \hookrightarrow G_{4}\right.\right.$, true $\left.)\right)$ (an extension of this graph property is given in Figure 12b) according to Def. 16 because the required morphisms $q_{2}$ and $q_{4}$ can be found such that the two triangles commute (see Figure 11 for an example with more explanations on the satisfaction of graph properties).

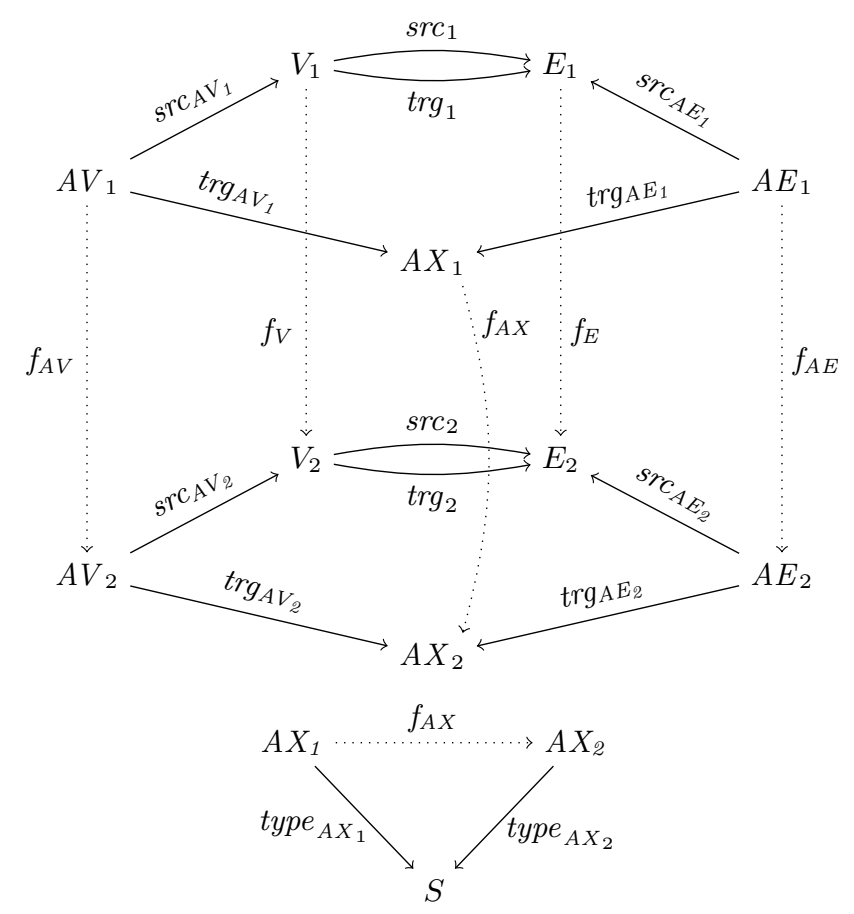

Figure 9: A symbolic attributed graph morphism $f$ (see Def. 11) has to be compatible with the operations of the source and target graphs.

\footnotetext{
Theorem 1 (Category GRAPHSSTA of Symbolic Typed Attributed Graphs). We obtain the category GRAPHSSTA by using the symbolic typed attributed graphs as defined in Def. 12 as objects, the symbolic typed attributed graph morphisms as defined in Def. 11 between them as morphisms, the composition of symbolic typed attributed graph morphisms also defined in Def. 11, and symbolic typed attributed graph identity morphisms based on componentwise identities.
}

Proof (idea). By the well-definedness of the involved notions of symbolic typed attributed graphs and symbolic typed attributed morphisms.

In the following we denote that $f: G \rightarrow G^{\prime}$ is a mono as $f: G \hookrightarrow G^{\prime}$, an epi as $f: G \rightarrow G^{\prime}$, and an iso as $f: G \hookrightarrow G^{\prime}$ (see Lem. 11).

Note, for specifications where attribute constraint satisfiability is undecidable we can also not decide whether an element is a graph morphism because, by definition, an implication on attribute constraint satisfaction must be decided. In section 8 we handle this problem explicitly for our symbolic model generation procedure.

\section{Properties over GRAPHSSTA}

In this section we introduce graph properties over symbolic typed attributed graphs with basic operations.

On the one hand, graph properties (for labelled graphs) with attribute constraints without nesting have been introduced in [32] and extended with operators from propositional logic in [33]. On the other hand, graph properties (for labelled graphs) without attribute constraints but with nesting have been introduced in [21]. In the following we integrate both ideas and introduce (nested) graph properties on symbolic typed attributed graphs from subsection 4.2.

Graph conditions of the simple form $\exists(m: \emptyset \hookrightarrow G$, true $)$ state that $G$ is to be contained as a subgraph in every model. The expressive power of first order logic on graphs is then obtained by allowing conjunction, negation, and nesting of graph conditions of this form.

Definition 15 (Graph Conditions and Graph Properties). The set $\mathcal{C}_{G}$ of (graph) conditions over a graph $G$ is inductively ${ }^{2}$ defined by:

\footnotetext{
2 The empty conjunction $\wedge \emptyset$ is the base case of the inductive definition.
} 
- $\wedge S \in \mathcal{C}_{G}$ if $S$ is a finite subset of $\mathcal{C}_{G}$,

- $\neg c \in \mathcal{C}_{G}$ if $c \in \mathcal{C}_{G}$, and

- $\exists\left(m: G \hookrightarrow G^{\prime}, c\right) \in \mathcal{C}_{G}$ if $c \in \mathcal{C}_{G^{\prime}}$.

A (graph) property is a condition over the empty graph $\emptyset$.

Notation 1. We use further operators to ease the usage of graph properties, introduced as abbreviations, such as

$$
\begin{aligned}
\vee S & :=\neg \wedge\{\neg c \mid c \in S\}, \\
c_{1} \rightarrow c_{2} & :=\vee\left\{\neg c_{1}, c_{2}\right\}, \\
\text { true } & :=\wedge \emptyset, \\
\text { false } & :=\vee \emptyset, \text { and } \\
\forall(m, c) & :=\neg \exists(m, \neg c) .
\end{aligned}
$$

We also allow infix and mixfix notation for $\wedge$ and $\vee$ (e.g., as in $\wedge\left\{c_{1}, c_{2}, c_{3}\right\}=c_{1} \wedge c_{2} \wedge c_{3}$ ).

When presenting graph conditions in figures, we abbreviate (for notational simplicity) existential quantifications of the form $\exists\left(m: G_{1} \hookrightarrow G_{2}, c\right)$ by $\exists\left(G^{\prime}, c\right)$ when the morphism $m$ is clear from the context and where $G^{\prime}$ is the least subgraph of $G_{2}$ containing all graph elements that are fresh in $G_{2}$ w.r.t. $G_{1}$ (used in, e.g., Figure 3).

Graph properties may be employed to specify a diverse set of properties to be satisfied by concrete models. Firstly, the graph property in Figure 10a states a simple negative pattern by means of the symbolic typed attributed graph $G^{7}$ from Figure 7. Secondly, the graph properties from Figure 10b, Figure 10d, and Figure 10c specify lower/upper bounds (i.e., multiplicity statements) on graph elements that are mapped to common graph elements in the type graph. Finally, the graph property given in Figure 10e describes an infinite sequence of nodes by essentially formalizing the Peano axioms. The last example also demonstrates that any step wise construction of minimal models adding a finite number of elements can not terminate in general.

Definition 16 (Satisfaction of Graph Conditions). A graph condition $c_{i n p}$ from $\mathcal{C}_{C}$ is satisfied recursively by a monomorphism $q: C \hookrightarrow G$, written $q \models c_{i n p}$, as follows:

$-q \models \wedge S$ if $q \models c$ for each $c \in S$

$-q \models \neg c$ if not $q \models c$

$-q \mid=\exists(m: C \hookrightarrow D, c)$ if some $q^{\prime}: D \hookrightarrow G$ satisfies $q^{\prime} \circ m=q$ and $q^{\prime} \models c$.

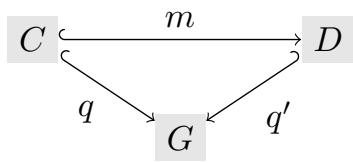

Also, a graph $G$ satisfies a graph property $p$ (see Def. 15), written $G \models p$, if the unique mono $i_{G}: \emptyset \hookrightarrow G$ satisfies $p$. Finally, $\llbracket p \rrbracket$ is the set of all graphs satisfying $p$ and two properties $p_{1}$ and $p_{2}$ are equivalent, written $p_{1} \equiv p_{2}$, iff $\llbracket p_{1} \rrbracket=\llbracket p_{2} \rrbracket$.

Consider Figure 11 for an example for the satisfaction of a graph property making use of nesting, conjunction, and existential quantification where types, attributes, attribute constraints, and negation are left out for simplicity.

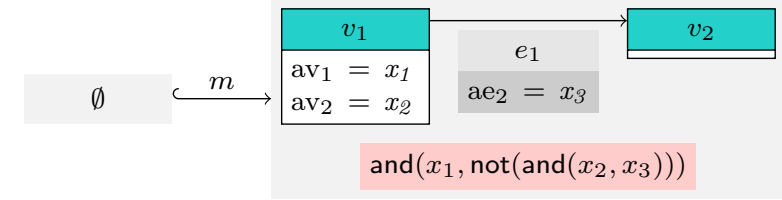

(a) Graph property $p^{10 a}=\neg \exists(m$, true $)$ states that the symbolic attributed graph $G^{7}$ from Figure 7 (equipped with suitable vertex and edge types not depicted for simplicity) is not a subgraph of any desired model. That is, the pattern described by $G^{7}$ can be understood to be prohibited.

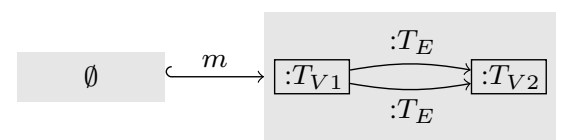

(b) Graph property $p^{10 b}=\neg \exists(m$, true $)$ states that there are no parallel edges of type $T_{E}$ between vertices of types $T_{V 1}$ and $T_{V 2}$, respectively.

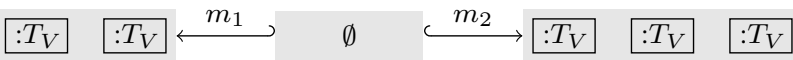

$$
\begin{aligned}
& p_{1}^{10 c}=\exists\left(m_{1}, \text { true }\right)
\end{aligned}
$$

(c) Graph property $p^{10 c}=p_{1}^{10 c} \wedge p_{2}^{10 c}$ states that $\left(p_{1}^{10 c}\right)$ there are at least two vertices of type $T_{V}$ and that $\left(p_{2}^{10 c}\right)$ there are not three or more vertices of type $T_{V}$.

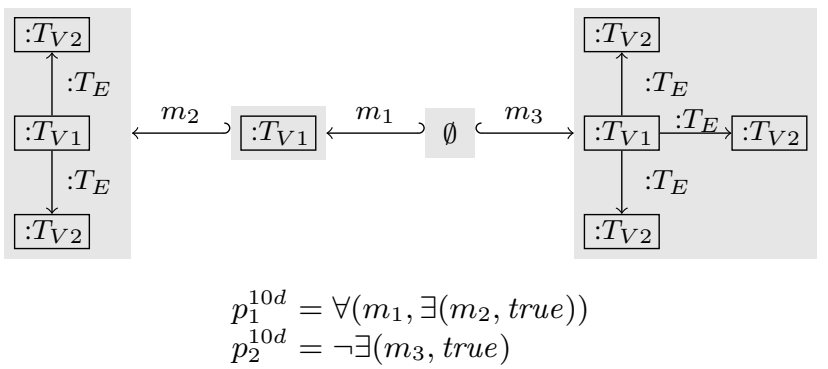

(d) Graph property $p^{10 d}=p_{1}^{10 d} \wedge p_{2}^{10 d} \wedge p^{10 b}$ states that $\left(p_{1}^{10 d}\right)$ at least two edges of type $T_{E}$ exit every vertex of type $T_{V 1}$, and that $\left(p_{2}^{10 d}\right)$ not three or more edges of type $T_{E}$ exit any vertex of type $T_{V 1}$, and ( $p^{10 b}$ from Figure $10 \mathrm{~b}$ ) is used to simplify the property (no mergings of target vertices need to be considered in the property) in contexts where preventing parallel edges is reasonable.

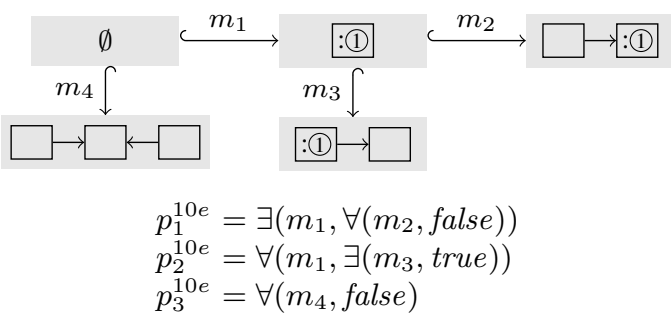

(e) Graph property $p^{10 e}=p_{1}^{10 e} \wedge p_{2}^{10 e} \wedge p_{3}^{10 e}$, with unique vertex and edge types (not depicted for simplicity), states that $\left(p_{1}^{10 e}\right)$ there is at least one vertex without predecessor vertex, $\left(p_{2}^{10 e}\right)$ every vertex has a successor vertex, and $\left(p_{3}^{10 e}\right)$ there is no vertex with two predecessor vertices. The infinite graph $\square \rightarrow \square \rightarrow \square \rightarrow \square \rightarrow \square \longrightarrow \cdots \quad$ is the least model of property $p^{10 e}$ and (an isomorphic copy of this graph) is contained in each model of $p^{10 e}$.

Figure 10: Various examples of graph properties showing the diversity of properties expressible by graph properties. 


$$
\begin{gathered}
p_{1}=\exists\left(m_{1}, p_{2}\right) \\
p_{2}=p_{3} \wedge p_{4} \\
p_{3}=\exists\left(m_{2}, \text { true }\right) \\
p_{4}=\exists\left(m_{3}, \text { true }\right) \\
G_{\text {host }}=v_{a} \cong v_{b} \odot v_{c} \odot
\end{gathered}
$$

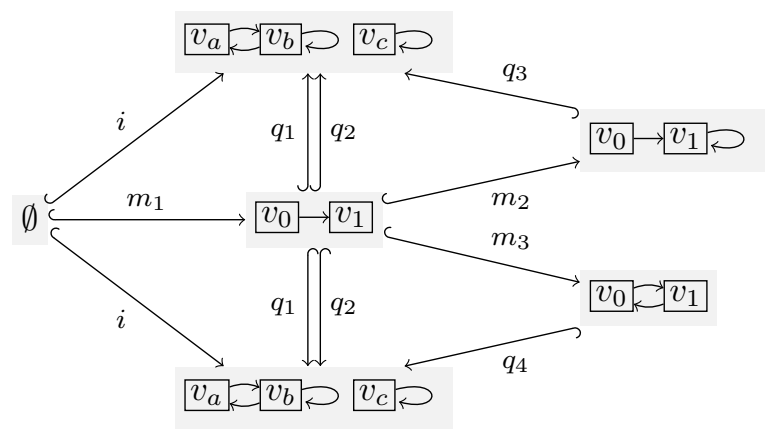

Figure 11: For the given graph property $p_{1}$ with its subconditions $p_{2}, p_{3}$, and $p_{4}$ we derive that the graph $G_{h o s t}$ satisfies $p_{1}$ as follows. Step 1: $G_{h o s t}$ satisfies $p_{1}$ because the unique mono $i: \emptyset \hookrightarrow G_{\text {host }}$ satisfies $p_{1}$. Step 2: $i$ satisfies $p_{1}$ because we can determine some $q_{1}$ (mapping $v_{0}$ to $v_{a}$ and $v_{1}$ to $v_{b}$ ) such that $q_{1} \circ m_{1}=i$ (trivial) and $q_{1}$ satisfies $p_{2}$. Step 3: $q_{1}$ satisfies $p_{2}$ because $q_{1}$ satisfies $p_{3}$ and $q_{1}$ satisfies $p_{4}$. Step 4a: $q_{1}$ satisfies $p_{3}$ because we can determine some $q_{3}$ (mapping $v_{0}$ to $v_{a}$ and $v_{1}$ to $v_{b}$ ) such that $q_{2} \circ m_{2}=q_{1}$ and $q_{2}$ satisfies true (trivial). Step 4b: $q_{1}$ satisfies $p_{4}$ because we can determine some $q_{4}$ (mapping $v_{0}$ to $v_{a}$ and $v_{1}$ to $v_{b}$ ) such that $q_{4} \circ m_{3}=q_{1}$ and $q_{4}$ satisfies true (trivial). Note, instead of choosing $q_{1}$ as above in Step 1 we could have selected $q_{2}$ (mapping $v_{0}$ to $v_{b}$ and $v_{1}$ to $v_{a}$ ). However, then we would have to derive that $q_{2}$ satisfies $p_{2}$. This is not possible because $q_{2}$ does not satisfy $p_{3}$ because there is no morphism $q_{3}^{\prime}$ with same type as $q_{3}$ such that $q_{3}^{\prime} \circ m_{2}=q_{2}$ because vertex $v_{a}$ has no loop.

For the tableau procedure in section 6 we introduce the construction shift as a modification of the corresponding construction from [14, Construction 3.12, p. 15] where shift is employed in the context of the analysis of $\mathcal{M}$-adhesive transformation systems. The operation from [14] is adapted here by requiring that all involved morphisms are monomorphisms as required for conditions and for satisfaction (Def. 15 and Def. 16).

While shift is defined homomorphically on conjunction and negation, for existential quantification all possible overlappings of two positive graph patterns are constructed to compute all situations in which both positive patterns are satisfied. In the following definition this means that the condition shift $\left(m_{1}, \exists\left(m_{2}, c\right)\right)$ describes the occurrence of the positive pattern $\exists\left(m_{2}, c\right)$ in the context of the other positive pattern $\exists\left(m_{1}\right.$, true $)$.

Consider Figure 12 for an example of a graph property with attribute constraints that is shifted along a monomorphism where overlappings are constructed and attribute constraints are handled suitably.

Definition 17 (Operation shift). Given a graph condition from $\mathcal{C}_{C}$ and a monomorphism $m_{1}: C \hookrightarrow C_{1}$. Then, shift is defined recursively as follows:

$$
\begin{aligned}
& \text { - } \operatorname{shift}\left(m_{1}, \wedge S\right)=\wedge\left\{\operatorname{shift}\left(m_{1}, c\right) \mid c \in S\right\}, \\
& \text { - } \operatorname{shift}\left(m_{1}, \neg c\right)=\neg \operatorname{shift}\left(m_{1}, c\right) \text {, and } \\
& \text { - } \operatorname{shift}\left(m_{1}, \exists\left(m_{2}: C \hookrightarrow C_{2}, c\right)\right) \\
& \quad=\vee\left\{\exists\left(m_{2}^{\prime}, \operatorname{shift}\left(m_{1}^{\prime}, c\right)\right) \mid\left(m_{2}^{\prime}, m_{1}^{\prime}\right) \in \mathcal{F}\right\} \text { where } \mathcal{F}
\end{aligned}
$$

is a set of representatives for the isomorphism quotient of $\left\{\left(m_{2}^{\prime}, m_{1}^{\prime}\right) \in \mathcal{E}^{\prime} \mid m_{2}^{\prime} \circ m_{1}=m_{1}^{\prime} \circ m_{2}\right\}$ where $\mathcal{E}^{\prime}$ is the set of pairs of jointly epimorphic morphisms from Def. 32 . Here $\left(m_{2}^{\prime}, m_{1}^{\prime}\right)$ and $\left(m_{2}^{\prime \prime}, m_{1}^{\prime \prime}\right)$ are isomorphic, if some

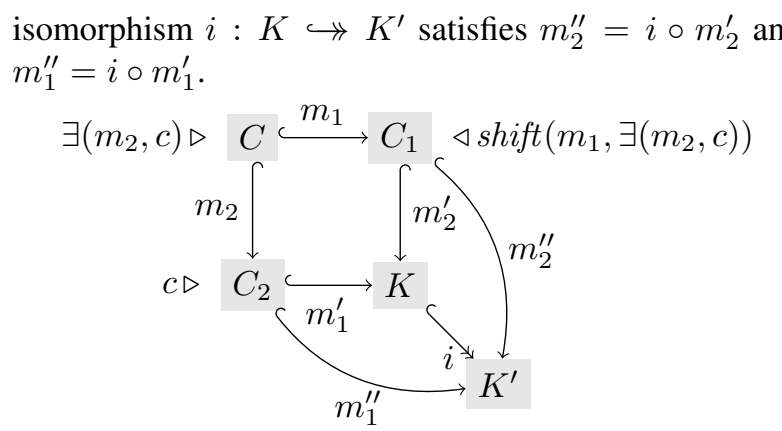

The definition of shift is of course important for the implementation. However, in proofs we only build upon the following lemma stating the required compatibility.

Lemma 1 (Compatibility of shift and $\models$ ). Given a graph condition $c \in \mathcal{C}_{C}$, a monomorphism $m: C \hookrightarrow C^{\prime}$, and a monomorphism $m^{\prime}: C^{\prime} \hookrightarrow G$. Then, $m^{\prime} \circ m \models c$ iff $m^{\prime}=$ $\operatorname{shift}(m, c)$.

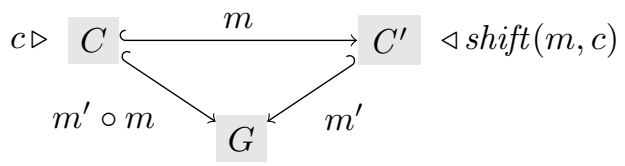

Proof (idea). Analogous to the proof of the corresponding earlier result [14, Lem. 3.11, p. 15] using Lem. 14.

To simplify our reasoning, our symbolic model generation algorithm operates on the subset of conditions in conjunctive normal form (CNF).

Definition 18 (Graph Conditions in Conjunctive Normal Form (CNF)). A graph condition is in CNF if it is a conjunction of clauses. A clause is a disjunction of literals. A literal is a positive literal $\exists(m, c)$ or a negative literal $\neg \exists(m, c)$ where (in both cases) $m: C \hookrightarrow C^{\prime}$ is no isomorphism, the attribute constraint of $C^{\prime}$ is satisfiable, and $c$ is in CNF. 


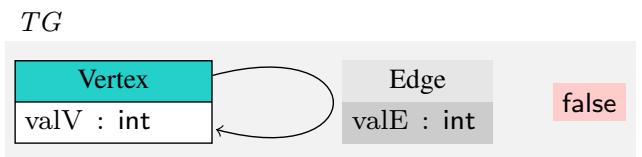

(a) The type graph $T G$ (used in this figure) over the specification from Def. 30.

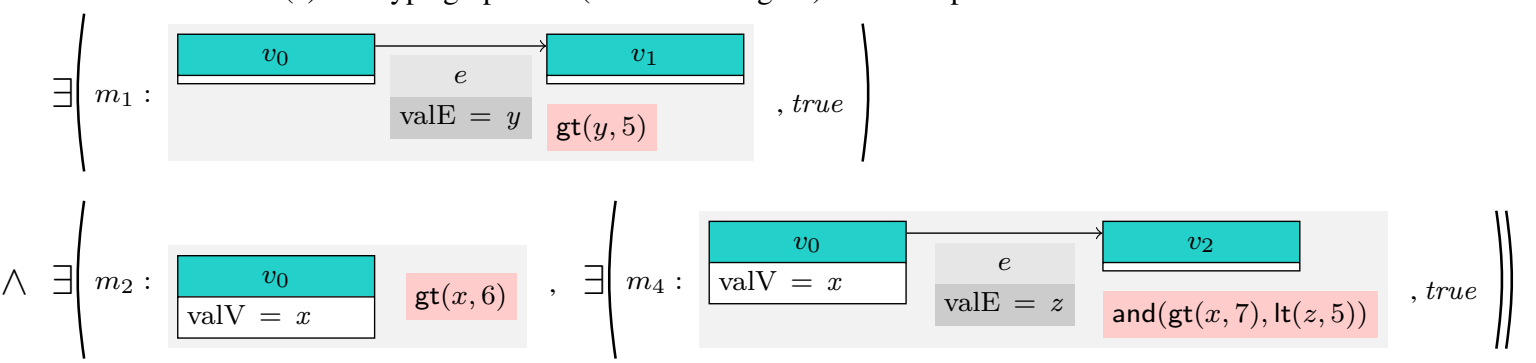

(b) A graph property $p=\exists\left(m_{1}: G_{0} \hookrightarrow G_{1}\right.$, true $) \wedge \exists\left(m_{2}: G_{0} \hookrightarrow G_{2}, \exists\left(m_{4}: G_{2} \hookrightarrow G_{4}\right.\right.$, true $\left.)\right)$, which is a condition over the empty graph $G_{0}=\emptyset$ by definition, where all graphs are typed over the type graph $T G$ from above.

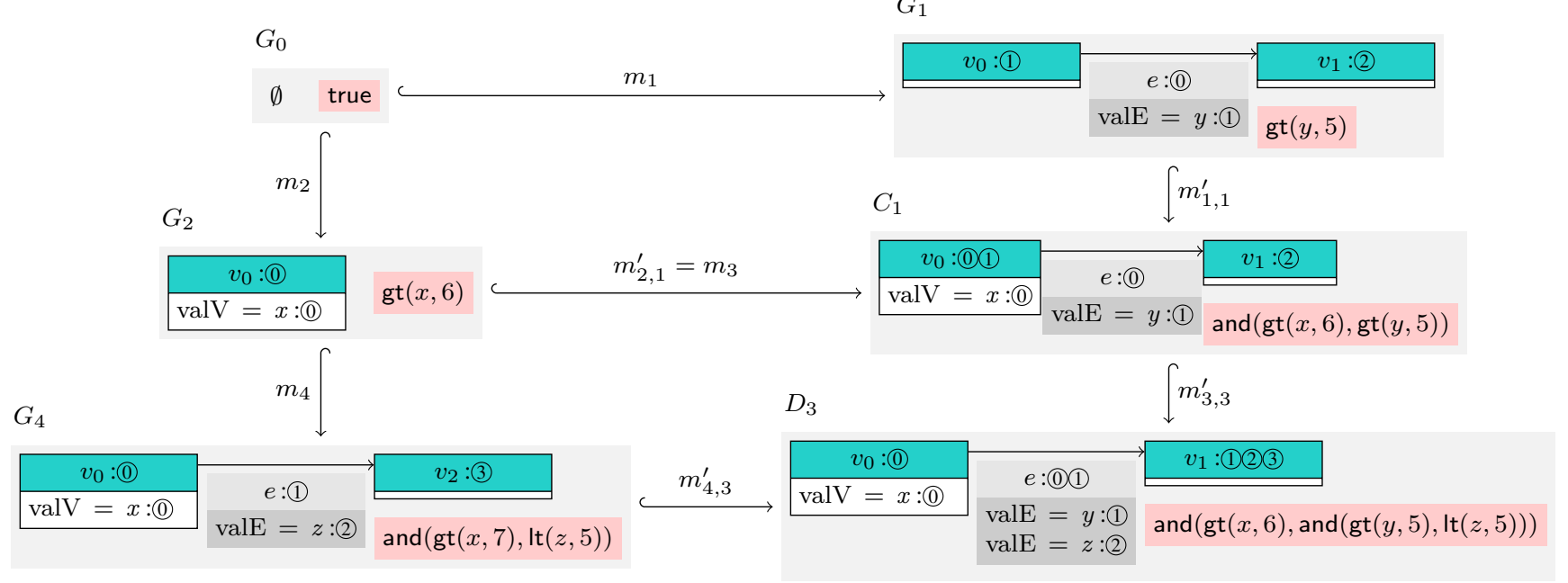

(c) One of the diagrams required for computing shift $\left(m_{1}: G_{0} \hookrightarrow G_{1}, \exists\left(m_{2}: G_{0} \hookrightarrow G_{2}, \exists\left(m_{4}: G_{2} \hookrightarrow G_{4}\right.\right.\right.$, true $\left.\left.)\right)\right)$ showing that the result (which is a disjunction) contains at least $\exists\left(m_{1}: G_{0} \hookrightarrow G_{1}, \exists\left(m_{1,1}^{\prime}: G_{1} \hookrightarrow C_{4}, \exists\left(m_{3,3}^{\prime}: C_{1} \hookrightarrow D_{3}\right.\right.\right.$, true $\left.)\right)$ ). Firstly, (in the upper rectangle) $C_{1}$ is constructed from $G_{1}$ and $G_{2}$ by some (possibly partial) overlapping and, secondly, (in the lower rectangle) $D_{3}$ is constructed from $C_{1}$ and $G_{4}$ by some (possibly partial) overlapping such that elements that are already in $G_{2}$ are overlapped. During the overlapping process the constraints of the result are the constraints of the two source graphs where variables are renamed according to the variable mappings from the source graphs into the constructed overlapping. Note, the graph $D_{3}$ satisfies the graph property $p$.

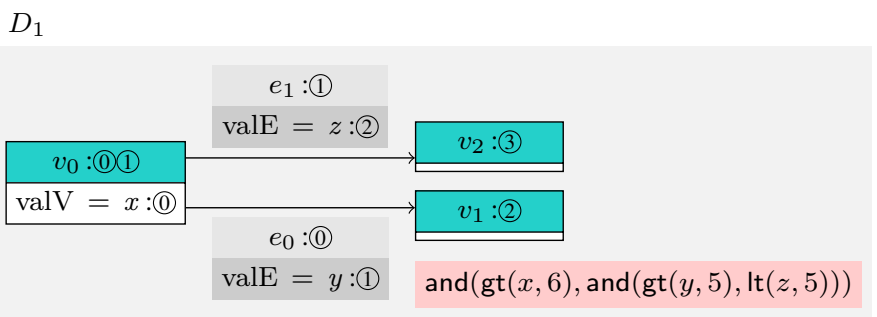

(d) An alternative overlapping to $D_{3}$.

$D_{4}$

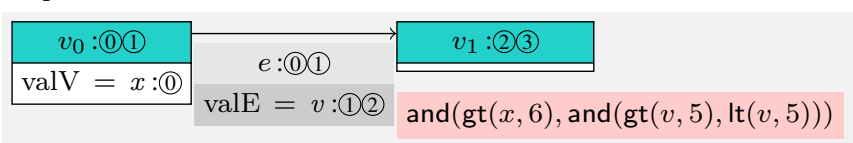

(f) An alternative overlapping to $D_{3}$ with unsatisfiable constraint.

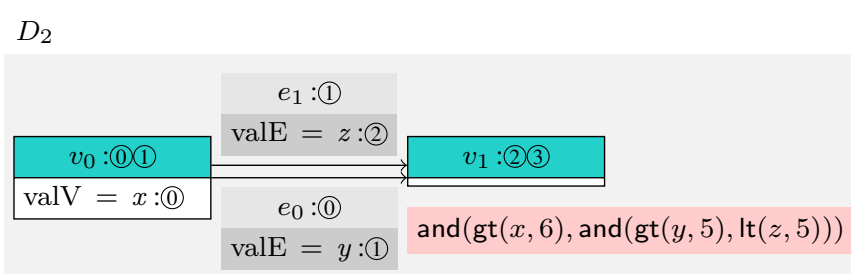

(e) An alternative overlapping to $D_{3}$.

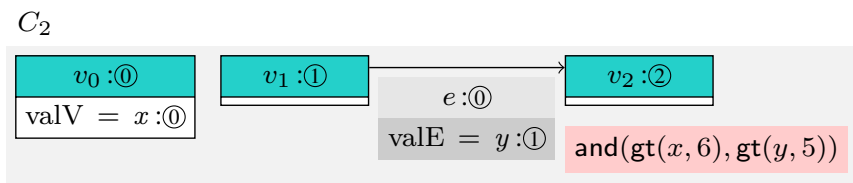

(g) An alternative overlapping to $C_{1}$.

Figure 12: An example of an application of the shift-operation. 
For example, the condition $\wedge\{\vee\{\}\}$ is in CNF and is equivalent to false.

For translating conditions into equivalent conditions in CNF we introduce the second operation [.] on conditions, which is similar to an operation in [38,39,27].

Definition 19 (Conversion to CNF). The conversion operation $[\cdot]: \mathcal{C}_{G} \rightarrow \mathcal{C}_{G}$ executes the following steps:

- Step 1: remove operators besides $\wedge, \vee, \neg$, and $\exists$ according to the abbreviations from Notation 1 ,

- Step 2: remove any existential quantifications of isomorphisms (e.g., $\exists(i: A \hookrightarrow B, \exists(m: B \hookrightarrow C$, true $))$ is replaced by $\exists((m \circ i): A \hookrightarrow C$, true $)$ by moving the isomorphism $i$ into the literal of the next nesting level),

- Step 3: remove all unsatisfiable existential quantifications (i.e., replace $\exists(m: A \hookrightarrow B, c)$ by $\vee \emptyset$ when the constraints of the graph $B$ are not satisfiable),

- Step 4: move all negations inwards across $\wedge$ and $\vee$, dropping duplicate negations as expected, until reaching an existential quantification, and

- Step 5: apply distributive and associative laws for $\wedge$ and $\checkmark$ to finally enforce the required CNF structure.

In Step 3 we require the existence of an oracle that decides these satisfiability questions in all cases. In section 8 we explain in more detail how we handle cases where SMT solvers such as Z3 designed to implement that oracle are unable to decide satisfiability questions on attribute constraints.

As for FOL the conversion to CNF entails the conversion of subconditions of the shape $\left(a_{1} \wedge b_{1}\right) \vee \cdots \vee\left(a_{n} \wedge b_{n}\right)$ resulting in $2^{n}$ clauses of size $n$. However, in our approach the conversion of graph conditions into CNF graph conditions usually has no great impact on the runtime of our overall algorithm because subconditions from different existential quantifiers are not combined in the conversion, that is, we perform the conversion on each nesting level of the $\exists$-quantifier and, hence, we obtain quite small CNF conditions. For FOL this is different: after skolemization, which removes existential quantifiers, all subconditions are related to each other resulting in huge formulas. Note, skolemization is not needed for graph conditions according to [39, p. 100]. Also note, the size of the graphs and the morphisms contained in the condition are not relevant for the conversion in our case, which is an important difference to the FOL scenario. However, attribute constraints are checked by AUTOGRAPH for satisfiability by the employed SMT solver during the conversion to CNF. This intuitive explanation is supported by runtime examinations presented in [47] where ALLOY is applied to generate models for two graph queries. However, apparently due to lacking support for strings and integer arithmetic, ALLOY is not able to determine models for ALLOY-encoded versions of the graph properties with attributes and attribute conditions from Figure 4, Figure 5, and Figure 6.

\section{Tableau Procedure}

In this section we adapt the tableau-procedure for graph conditions without attributes and attribute constraints from [27] to the symbolic typed attributed graphs as introduced in subsection 4.2. Due to the additional attributes and attribute constraints underlying operations such as shift and [.] have been adapted as explained before.

Intuitively, the tableau procedure performs, for a given graph property, a recursive case distinction to finally return all most-explicit cases that can not be split further. Subsequently, in subsection 6.1, we start with an intuitive explanation on the steps for splitting, recursive application, and termination backed up by fundamental lemmas. Afterwards, in subsection 6.2, we present formal definitions for the construction of (Nested) Tableaux implementing the steps explained before.

\subsection{Recursive Case Distinction Principle}

Step 1 (Splitting): we are translating the given graph condition $c$ in $\mathrm{CNF}$ into a disjunctive normal form, i.e., into a disjunction of conjunctions of literals. This conversion is executed in subsection 6.2 by construction of a tableau $T$ where each branch $B$ of the tableau $T$ corresponds to one clause of the disjunctive normal form to be constructed.

The obtained condition $\vee S$ is then considered compositionally (assuming here and subsequently an enclosing existential quantification in the form of $\exists\left(i_{C}, \vee S\right)$ using the unique mono $i_{C}: \emptyset \hookrightarrow C$ ). That is, we can consider each clause of the disjunctive normal form (given by an element of $S$ ) separately without altering the set of models of the graph property. Hence, we may consider one branch $B$ of the tableau $T$ constructed in isolation.

Lemma 2 (Sound and Complete Branching). If $S$ is a subset of $\mathcal{C}_{C}$, then $\llbracket \exists\left(i_{C}, \vee S\right) \rrbracket=\bigcup\left\{\llbracket \exists\left(i_{C}, c\right) \rrbracket \mid c \in S\right\}$.

Each clause $\wedge L$ now considered separately from the others either contains a positive literal (Step 2) or only negative literals (Step 3).

Step 2 (Recursive Application): To prepare the clause $\wedge L$ for recursive application we are merging the elements of $L$ into a single positive literal by application of the shift construction. Firstly, one positive literal $\ell$ is selected from $L$ and all other graph conditions from $S=L-\{\ell\}$ are lifted into $\ell$ using the shift construction.

Lemma 3 (Sound and Complete Lifting). If $S$ is a given subset from $\mathcal{C}_{C}$, then $\llbracket \exists\left(i_{C},(\wedge S) \wedge \exists\left(m: C \hookrightarrow C^{\prime}, c^{\prime}\right)\right) \rrbracket=$ $\llbracket \exists\left(i_{C}, \exists\left(m, c^{\prime} \wedge \operatorname{shift}(m, \wedge S)\right)\right) \rrbracket$.

Also note, the operation [.], which is additionally applied in the formal tableau construction in subsection 6.2 , is sound and complete as follows.

\section{Lemma 4 (Sound and Complete $[\cdot]$ ). \\ If $c$ is a condition from $\mathcal{C}_{C}$, then $\llbracket \exists\left(i_{C}, c\right) \rrbracket=\llbracket \exists\left(i_{C},[c]\right) \rrbracket$.}

Finally, we recursively apply the presented algorithm to the condition $c$ of the positive literal $\exists\left(m: C \hookrightarrow C^{\prime}, c\right)$ obtained. Note, since $m$ is no isomorphism due to the operation [.] the graph $C^{\prime}$ is strictly greater than the graph $C$. This recursive application is justified by the following lemma. 
Lemma 5 (Sound and Complete Nesting). If $c$ is a condition in $\mathcal{C}_{C^{\prime}}$, then $\llbracket \exists\left(i_{C}, \exists\left(m: C \hookrightarrow C^{\prime}, c\right)\right) \rrbracket=\llbracket \exists\left(i_{C^{\prime}}, c\right) \rrbracket$.

Step 3 (Termination): As a complementary case to Step 2 we consider clauses containing no positive literal. That is, clauses containing any number of negative literals of the form $\neg \exists\left(m: C \hookrightarrow C^{\prime}, c\right)$. Due to the construction of the operation [.] the monomorphisms $m$ of these negative literals are no isomorphisms and, hence, the unique monomorphism $i_{C}: \emptyset \hookrightarrow C$ already satisfies these negative literals proving that $C$ is a model of all negative literals contained in the clause. Also, the graph $C$ minimally represents all models of the considered case in the sense that it is the least graph contained in all these models.

Lemma 6 (Sound and Complete Termination). Let $L$ be a set of negative literals $\neg \exists\left(m_{i}, c_{i}\right)$ from $\mathcal{C}_{C}$ where each $m_{i}$ is no isomorphism. Then $C$ is the unique least element of $\llbracket \exists\left(i_{C}, \wedge L\right) \rrbracket$ in the sense of

- existence: $C$ is an element of $\llbracket \exists\left(i_{C}, \wedge L\right) \rrbracket$

- unique least: for each graph $C^{\prime} \in \llbracket \exists\left(i_{C}, \wedge L\right) \rrbracket$ there exists some monomorphism $m: C \hookrightarrow C^{\prime}$

The algorithm terminates at this point with conditions of the form $\llbracket \exists\left(i_{C}, \wedge L\right) \rrbracket$, which can not be broken down using a case distinction as mentioned before and which are called therefore most-explicit.

Note, the proofs of the lemmas above are contained in the appendix.

Subsequently we formalize the presented description of the algorithm by introducing definitions for the construction of tableaux and, for recursive application, nested tableaux.

\subsection{Recursive Construction of Tableaux}

The algorithm intuitively presented before is now formalized by means of the tableau based reasoning method as introduced in [27]. Regular tableaux are used to perform the splitting in Step 1 from above and were directly inspired by the construction of tableaux for plain FOL reasoning [22]. Then, nested tableaux are used to handle the recursive application of Step 2 from above.

Provided a condition in CNF and an empty tableau obtained using the initialization rule we are using the extension rule to construct branches by selecting one literal from each clause (note, a condition is unsatisfiable if it contains an empty clause). Then, using the lift rule we are merging all literals of a branch into a single positive literal provided the branch contains at least one positive literal as a starting point.

\section{Definition 20 (Tableaux for Graph Conditions, Open and} Closed Branches). Given a condition $c$ in CNF over $C$. A tableau $T$ for $c$ is a finite tree whose nodes are conditions constructed using the rules below. A branch in a tableau $T$ for $c$ is a maximal path in $T$. Moreover, a branch $B$ is closed if false is in B; otherwise, it is open. Finally, a tableau is closed if all of its branches are closed; otherwise, it is open.
- initialization rule: a tree with a single root node true is a tableau for $c$.

- extension rule: if $B$ is a branch of a tableau for $c$ and $\vee S$ is a clause in $c$, then if $S \neq \emptyset$ and $S \cap B=\emptyset$, then append each element of $S$ as a child node to the leaf of $B$ or if $S=\emptyset$ and false $\notin B$, then append false as a child node to the leaf of $B$.

- lift rule: if $B$ is a branch of a tableau for $c, \ell$ and $\exists\left(m, c^{\prime}\right)$ are in $B, \ell^{\prime}=\exists\left(m,\left[c^{\prime} \wedge \operatorname{shift}(m, \ell)\right]\right)$ is not in $B$, then append $\ell^{\prime}$ as a child node to the leaf of $B$.

Semi-saturated tableaux are the desired results of the iterative tableaux construction where no further rules may be applied.

Definition 21 (Semi-saturated (Branch of a) Tableau). Let $T$ be a tableau for condition $c$ over $C$. A branch $B$ of $T$ is semi-saturated if it is either closed or

- $B$ is not extendable with a new node using the extension rule and

- if $E=\left\{\ell_{1}, \ldots, \ell_{n}\right\}$ is the nonempty set of literals contained in nodes added to $B$ using the extension rule (i.e., not by the lift rule), then there is a positive literal $\ell=$ $\exists\left(m, c^{\prime}\right)$ in $E$ such that the literal in the leaf node of $B$ is equivalent to $\exists\left(m, c^{\prime} \wedge\left\{\operatorname{shift}\left(m, \ell^{\prime}\right) \mid \ell^{\prime} \in(E-\{\ell\}\}\right)\right.$. Also, we call $\ell$ the hook of $B$.

Finally, $T$ is semi-saturated if all branches of $T$ are semisaturated.

Note, the set $E$ in the definition above contains all literals that are to be integrated using the lift rule in the leaf node of $B$.

Following the description of the algorithm from before we recursively construct further tableaux for the inner conditions $c^{\prime}$ of the leaf nodes $\exists\left(m, c^{\prime}\right)$ contained in the tableau at hand. That is, the next analysis step is to construct a tableau for condition $c^{\prime}$. The iterative (possibly non-terminating) execution of this procedure results in (possibly infinitely many) tableaux where each tableau may result in the construction of a finite number of further tableaux. This relationship between a tableau and the tableaux derived from the leaf literals of open branches results in a so called nested tableau (see Figure 13 for an example of a nested tableau).

Definition 22 (Nested Tableaux, Opener of Tableau, Context of Tableau, Nested Branch of Nested Tableau, Semisaturated Nested Tableau). Given a condition $c$ over $C$ and a partially ordered set $\left(I, \leq, i_{0}\right)$ with minimal element $i_{0}$. A nested tableau NT for $c$ is constructed using the rules below and is, for some $I^{\prime} \subseteq I$, a family ${ }^{3}$ of triples $\left\{\left\langle T_{i}, j, c_{j}\right\rangle\right\}_{i \in I^{\prime}}$ that contain a tableau $T_{i}$, an index $j \in I^{\prime}$, and a condition $c_{j}{ }^{4}$

- initialization rule: If $T_{i_{1}}$ is a tableau for $c$, then the family containing only $\left\langle T_{i_{1}}, i_{0}\right.$, true $\rangle$ for some index $i_{1}>i_{0}$ is a nested tableau for $c$ and $C$ is called context of $T_{i_{1}}$.

\footnotetext{
3 Formally, the family is a map that assigns one triple to each $i \in I^{\prime}$.

4 Intuitively, a triple $\left\langle T_{i}, j, c_{i}\right\rangle$ is either generated by the initialization rule or is generated by the nesting rule and $T_{i}$ is a tableau for a condition $c_{i}$ that is the inner condition of some literal $\ell=\exists\left(m, c_{i}\right)$ that is in a leaf node of the parent tableau $T_{j}$ that is assigned to index $j$ in $N T$.
} 


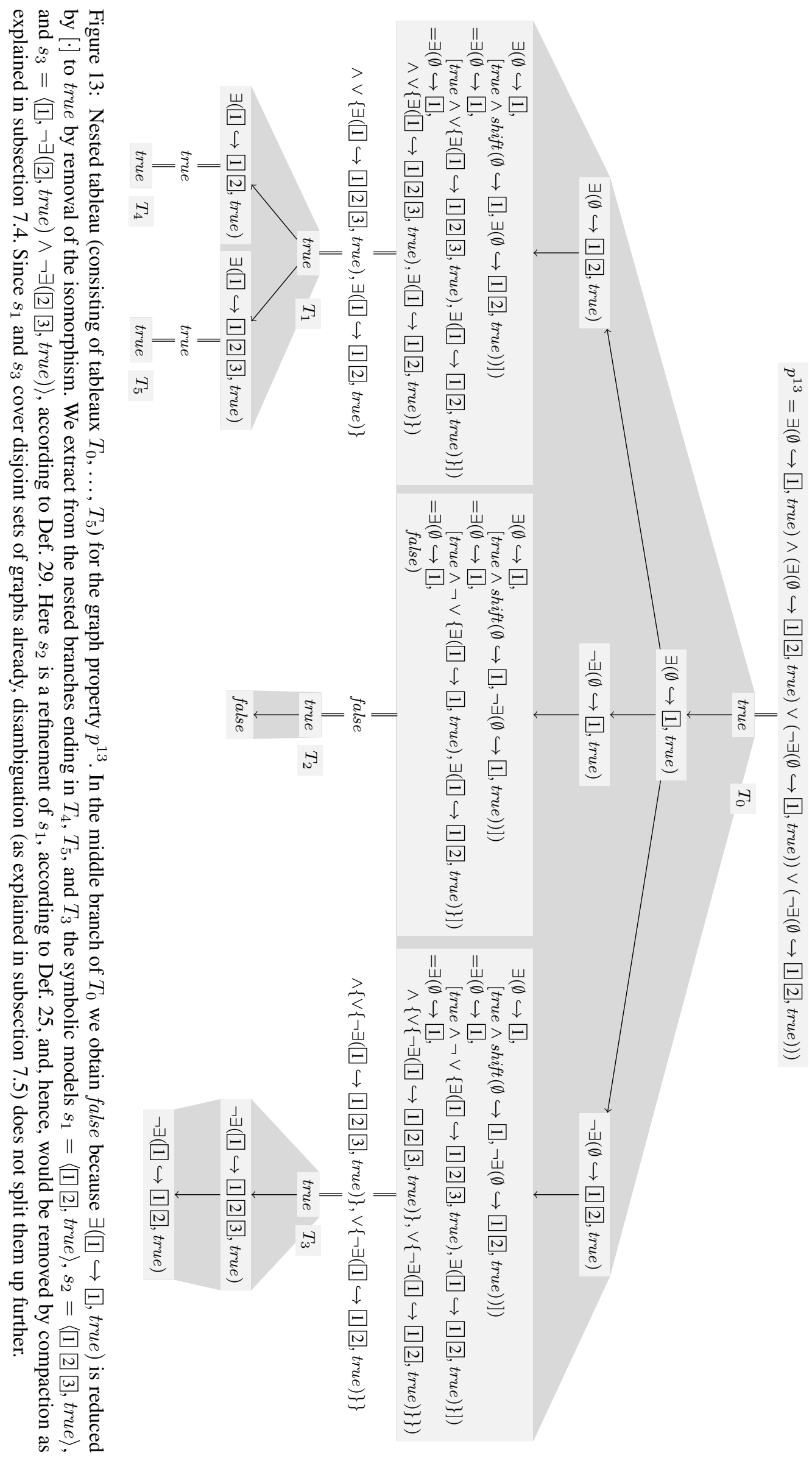


- nesting rule: If $N T$ is a nested tableau for $c$ with index set $I^{\prime},\left\langle T_{n}, k, c_{k}\right\rangle$ is in $N T$ for index $n$, the literal $\ell=\exists\left(m_{n}\right.$ : $\left.A_{n} \hookrightarrow A_{j}, c_{n}\right)$ is a leaf of $T_{n}, \ell$ is not the condition in any other triple of $N T, T_{j}$ is a tableau for $c_{n}$, and $j>n$ is some index not in $I^{\prime}$, then assign the triple $\left\langle T_{j}, n, \ell\right\rangle$ to $N T$ to index $j, \ell$ is called opener of $T_{j}$, and $A_{j}$ is called context of $T_{j}$.

A nested branch $N B$ of the nested tableau $N T$ is a maximal sequence of branches $B_{i_{1}}, \ldots, B_{i_{k}}, B_{i_{k+1}}, \ldots$ of the tableaux $T_{i_{1}}, \ldots, T_{i_{k}}, T_{i_{k+1}}, \ldots$ in $N T$ starting with a branch $B_{i_{1}}$ in the initial tableau $T_{i_{1}}$ of $N T$, such that if $B_{i_{k}}$ and $B_{i_{k+1}}$ are consecutive branches in the sequence then the leaf of $B_{i_{k}}$ is the opener of $T_{i_{k+1}} . N B$ is closed if it contains a closed branch; otherwise, it is open. NT is closed if all its nested branches are closed. Finally, NT is semi-saturated if each tableau in NT is semi-saturated.

The definitions for the construction of the (nested) tableaux above correspond closely to the ones in [46] as expected because they operate on the categorical level. This also implies that only the operations shift and [.] occurring in the definitions above require additional attention (see Lem. 1 and Lem. 4).

In addition to semi-saturation we require the notion of a saturated nested tableaux, which requires (informally) that all tableaux of the given nested tableau are semi-saturated and that hooks are selected in a fair way not postponing indefinitely the influence of a positive literal for detecting inconsistencies leading to closed nested branches.

It has been shown in [27] that the tableau based reasoning method using nested tableaux for conditions $c$ is sound and refutationally complete. In particular, soundness means that if we are able to construct a nested tableau where all its branches are closed then the original condition $c$ is unsatisfiable. Refutational completeness means that if a saturated nested tableau includes an open branch, then the original condition is satisfiable. In fact, each open finite or infinite branch in such a nested tableau defines a finite or infinite model of the property, respectively. Incompleteness can be caused in tableaux for FOL by unfair selection of formulas (confer [22, page 117, Figure 4] for an example where the unsatisfiable condition $Q \wedge \neg Q$ is treated unfairly by never being selected). In our case the set of conditions from which a hook is to be selected in a fair way changes from one tableau to the next because conditions that are not selected are lifted into the hook resulting (possibly) in multiple different conditions. These conditions are called the successors (cf. [27]) of the conditions that are selected and lifted. To ensure refutational completeness we ensure that the impact of a condition affects the nested branch eventually by not postponing the selection of one these successors as a hook indefinitely. Confer to [27, p. 29] for the discussion in the original paper.

However, recall again that the usage of attributes and attribute constraints in the graphs contained in the conditions leads to situations where the employed SMT solvers can not decide satisfiability. This has an impact on the construction of tableaux because the operation [.], as explained before, em- ploys SMT solvers to check satisfiability and it is applied in the lift rule in Def. 20.

\section{Symbolic Model Generation}

In this section we present our symbolic model generation algorithm $\mathcal{A}$. We first formalize the requirements from the introduction for the generated set of symbolic models, then present our algorithm, and subsequently verify that it indeed adheres to these formalized requirements. In particular, we want our algorithm to extract symbolic models from all open finite branches in a saturated nested tableau constructed for a graph property $p$.

Since there are infinite saturated nested tableaux, such as the one that would be constructed for the graph property $p^{10 e}$ given in Figure 10e, we have an incomplete procedure in the sense that the gradual construction of a nested tableau for a graph property $p$ may not terminate. However, due to the undecidability of FOL on graphs, no alternative sound and complete algorithm could also accomplish termination. In order to be able to find a complete set of symbolic models without knowing beforehand if the construction of a saturated nested tableau terminates, we introduce the key-notions of $k$-semisaturation and $k$-termination to reason about nested tableaux up to depth $k$, which are in some sense a prefix of a saturated nested tableau. Note, the verification of our algorithm, in particular for completeness, is accordingly based on induction on $k$. Informally, this means that by enlarging the depth $k$ during the construction of a saturated nested tableau, we eventually find all finite open branches and thus finite models. This procedure will at the same time guarantee that we will be able to extract symbolic models from finite open branches even for the case of an infinite saturated nested tableau. For example, we will be able to extract the graph $\square$ with a single vertex from a finite open branch of the infinite saturated nested tableau for property $p_{1}^{10 e} \vee p^{10 e}$.

\subsection{Sets of symbolic models}

The symbolic model generation algorithm $\mathcal{A}$ should generate for each graph property $p$ a set of symbolic models $\mathcal{S}$ satisfying all requirements described in the introduction (i.e., soundness, completeness, minimal representability, compactness, and nonambiguity). A symbolic model in its most general form is a graph condition over a graph $C$, where $C$ is available as an explicit component. A symbolic model then represents a possibly empty set of graphs (as defined subsequently in Def. 24).

Definition 23 (Symbolic Model, Remainder). If $c$ is a condition over $C$ according to Def. 15 , then $\langle C, c\rangle$ is a symbolic model. The condition $c$ is called remainder of the symbolic model.

We define the graphs that are covered by a given symbolic model as follows. 
Definition 24 (Graphs Covered by a Symbolic Model). If $\langle C, c\rangle$ is a symbolic model, then covered $(\langle C, c\rangle)$ is equal to $\llbracket \exists\left(i_{C}, c\right) \rrbracket$, i.e., the models of $\exists\left(i_{C}, c\right)$. For a set $\mathcal{S}$ of symbolic models covered $(\mathcal{S})=\bigcup\{\operatorname{covered}(s) \mid s \in S\}$.

Also note, each graph $G$ that is covered by a given symbolic model $\langle C, c\rangle$ subsumes the graph $C$ by means of some monomorphism as stated in the following lemma.

Lemma 7 (Existence of the Covering Monomorphism). If $\langle C, c\rangle$ is a symbolic model and $G \in \operatorname{covered}(\langle C, c\rangle)$, then there is some monomorphism $m: C \hookrightarrow G$.

For later use we also define when one symbolic model is entirely subsumed by another w.r.t. the covered graphs.

Definition 25 (Refinement of Symbolic Model). Given two symbolic models $\left\langle C_{1}, c_{1}\right\rangle$ and $\left\langle C_{2}, c_{2}\right\rangle$ s.t. $\llbracket \exists\left(i_{C_{2}}, c_{2}\right) \rrbracket \subseteq$ $\llbracket \exists\left(i_{C_{1}}, c_{1}\right) \rrbracket$, then $\left\langle C_{2}, c_{2}\right\rangle$ is a refinement of $\left\langle C_{1}, c_{1}\right\rangle$, written $\left\langle C_{2}, c_{2}\right\rangle \leq\left\langle C_{1}, c_{1}\right\rangle$. The set of all such symbolic models $\left\langle C_{2}, c_{2}\right\rangle$ is denoted by refined $\left(\left\langle C_{1}, c_{1}\right\rangle\right)$.

Based on these definitions, we formalize the first five requirements (that is, except for explorability) from section 1 to be satisfied by the sets of symbolic models returned by algorithm $\mathcal{A}$.

Definition 26 (Soundness, Completeness, Minimal Representability, Compactness, and Nonambiguity). Let $\mathcal{S}$ be a set of symbolic models and let $p$ be a graph property.

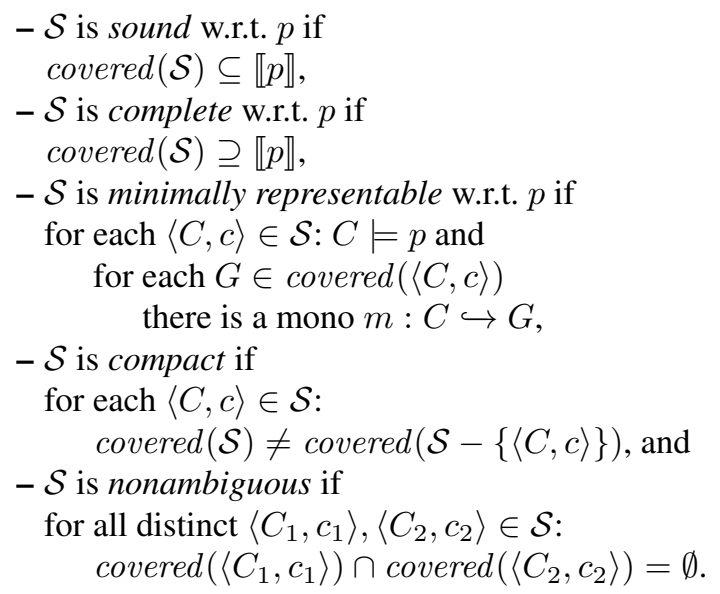

See Table 1 for distinguishing examples for compactness and nonambiguity when considering, for simplicity, graph part and attribute constraints in isolation. In subsection 7.4 and subsection 7.5 how both properties can be enforced, respectively. Subsequently we discuss the generation of sets of symbolic models by algorithm $\mathcal{A}$.

\subsection{Symbolic model generation algorithm $\mathcal{A}$}

We briefly describe the three main steps of the algorithm $\mathcal{A}$, which generates for a graph property $p$ a set of symbolic models $\mathcal{A}(p)$ (see Figure 14 for a visualization). The algorithm consists of three steps: the generation of symbolic models and the (optional) compaction and disambiguation of symbolic

\begin{tabular}{l|l}
\hline \multicolumn{1}{c}{ Symbolic model } & Edge-free graphs covered by $s_{i}$ \\
\hline$s_{0}=\langle\square \square$, true $\rangle$ & $\square \square, \square \square \square, \square \square \square \square, \ldots$ \\
$s_{1}=\langle\square \square \square$, true $\rangle$ & $\square \square \square, \square \square \square \square, \ldots$ \\
$s_{2}=\langle\square, \wedge\{\neg \exists(\square \square \square$, true $)\}\rangle$ & $\square, \square \square$ \\
$s_{3}=\langle\square, \wedge\{\neg \exists(\square \square$, true $)\}\rangle$ & $\square$ \\
\hline \hline \multicolumn{1}{c}{ Set of symbolic models } & Properties of $\mathcal{S}_{i}$ \\
\hline $\mathcal{S}_{1}=\left\{s_{0}, s_{1}\right\}$ & not compact, ambiguous \\
$\mathcal{S}_{2}=\left\{s_{0}, s_{2}\right\}$ & compact, ambiguous \\
$\mathcal{S}_{3}=\left\{s_{0}, s_{3}\right\}$ & compact, nonambiguous \\
\hline
\end{tabular}

(a) In the upper part of the table four symbolic models are given where we assume that the attribute constraint sets of the graphs are empty. For a better understanding we included some of the graphs without edges covered by the symbolic models. In the lower part of the table three sets of symbolic models are given with varying properties w.r.t. compactness and nonambiguity. $\mathcal{S}_{1}$ and $\mathcal{S}_{2}$ are ambiguous because both of their symbolic models cover the graph $\square \square \square$ and $\square \square$, respectively. $\mathcal{S}_{3}$ is nonambiguous because $s_{3}$ forbids two vertices while $s_{0}$ requires two vertices. $\mathcal{S}_{1}$ is not compact because each graph covered by $s_{1}$ contains two vertices as required by $s_{0} . \mathcal{S}_{2}$ is compact because, firstly, $s_{0}$ covers $\square \square \square$ while $s_{2}$ does not and, secondly, $s_{2}$ covers $\square$ while $s_{1}$ does not. $\mathcal{S}_{3}$ is compact because, firstly, $s_{0}$ covers $\square \square \square$ while $s_{3}$ does not and, secondly, $s_{3}$ covers $\square$ while $s_{0}$ does not.

\begin{tabular}{ll}
\hline \multicolumn{1}{c}{ Symbolic model } & Constraint set $\Phi_{i}$ of $C_{i}$ \\
\hline$s_{0}=\left\langle C_{1}, \wedge \emptyset\right\rangle$ & $\{\operatorname{ge}(x, 2)\}$ \\
$s_{1}=\left\langle C_{2}, \wedge \emptyset\right\rangle$ & $\{\operatorname{ge}(x, 3)\}$ \\
$s_{2}=\left\langle C_{3}, \wedge \emptyset\right\rangle$ & $\{\operatorname{le}(x, 2)\}$ \\
$s_{3}=\left\langle C_{4}, \wedge \emptyset\right\rangle$ & $\{\operatorname{lt}(x, 2)\}$ \\
\hline \hline
\end{tabular}

\begin{tabular}{l|l}
\hline \hline Set of symbolic models & Properties of $\mathcal{S}_{i}$ \\
\hline $\mathcal{S}_{1}=\left\{s_{0}, s_{1}\right\}$ & not compact, ambiguous \\
$\mathcal{S}_{2}=\left\{s_{0}, s_{2}\right\}$ & compact, ambiguous \\
$\mathcal{S}_{3}=\left\{s_{0}, s_{3}\right\}$ & compact, nonambiguous \\
\hline
\end{tabular}

(b) In the upper part of the table four symbolic models are given where we assume that the graphs $C_{i}$ share a common graph part containing an attribute-variable $x$ but differ in their attribute constraint sets $\Phi_{i}$. In the lower part of the table three sets of symbolic models are given with varying properties w.r.t. compactness and nonambiguity. $\mathcal{S}_{1}$ and $\mathcal{S}_{2}$ are ambiguous because both of their symbolic models allow $x$ to be 3 and 2 , respectively. $\mathcal{S}_{3}$ is nonambiguous because $s_{3}$ forbids $x$ to be 2 or greater while $s_{0}$ requires $x$ to be at least $2 . \mathcal{S}_{1}$ is not compact because each $x$ satisfying $\Phi_{1}$ is at least 2 as required by $s_{0} . \mathcal{S}_{2}$ is compact because, firstly, $s_{0}$ allows $x$ to be 3 while $s_{2}$ does not and, secondly, $s_{2}$ allows $x$ to be 1 while $s_{1}$ does not. $\mathcal{S}_{3}$ is compact because, firstly, $s_{0}$ allows $x$ to be 3 while $s_{3}$ does not and, secondly, $s_{3}$ allows $x$ to be 1 while $s_{0}$ does not.

Table 1: Two examples demonstrating compactness and nonambiguity of sets of symbolic models. The first example on the top considers symbolic models with different graphs with empty sets of attribute constraints and the second example on the bottom considers symbolic models with identical graph parts but differing attribute constraints. However, both examples are quite similar because the integer values occurring in the second example correspond to the number of vertices in the first example. 
models, which are discussed in detail in subsection 7.3, subsection 7.4, and subsection 7.5. Afterwards, in subsection 7.6, we discuss the explorability of the obtained set of symbolic models.

Step 1 (Generation of symbolic models in subsection 7.3). We apply the tableau and nested tableau rules from section 4 to iteratively construct a nested tableau for the given graph property $p$. Then, symbolic models are extracted from certain nested branches of this nested tableau that can not be extended. Since the construction of the nested tableau may not terminate due to infinite nested branches we construct the nested tableau in breadth-first manner and extract the symbolic models whenever possible. Moreover, to eliminate a source of nontermination we select the hook in each branch in a fair way not postponing the successors of a positive literal that was not chosen as a hook yet indefinitely [27, p. 29] ensuring at the same time refutational completeness of our algorithm. This step ensures that the resulting set of symbolic models is sound, complete (provided termination), and minimally representable. The symbolic models extracted from the intermediately constructed nested tableau $N T$ for growing $k$ is denoted $\mathcal{S}_{N T, k}$.

Step 2 (Compaction of sets of symbolic models in subsection 7.4). We remove all symbolic models from $\mathcal{S}_{N T, k}$ resulting in $\mathcal{S}_{\text {comp }}$ that do not contribute to the set of graphs jointly covered thereby enforcing compactness. This second step of our algorithm $\mathcal{A}$ preserves soundness, completeness, and minimal representability, and additionally ensures compactness.

Step 3 (Disambiguation of sets of symbolic models in subsection 7.5). We split the set $\mathcal{S}_{\text {comp }}$ of symbolic models obtained before resulting in $\mathcal{S}_{\text {res }}$ such that the graphs that are covered by the symbolic models from $\mathcal{S}_{\text {comp }}$ do not overlap pairwise, thereby enforcing nonambiguity. This third step of our algorithm $\mathcal{A}$ preserves soundness, completeness, minimal representability, and compactness and additionally ensures nonambiguity.

\subsection{Generation of $\mathcal{S}_{N T, k}$}

By applying a breadth-first construction we construct nested tableaux that are for increasing $k$, both, $k$-semi-saturated (i.e., all branches occurring up to index $k$ in all nested branches are semi-saturated), and $k$-terminated (i.e., no nested tableau rule can be applied to a leaf of a branch occurring up to index $k$ in some nested branch).

Definition 27 ( $k$-Semi-saturated Nested Branches, $k$-Terminated Nested Branches). Given a nested tableau $N T$ for condition $c$ over $C$. If $N B$ is a nested branch of length $k$ of $N T$ and each branch $B$ contained at index $i \leq k$ in $N B$ is semi-saturated, then $N B$ is $k$-semi-saturated. If every nested branch of $N T$ of length $n$ is $\min (n, k)$-semi-saturated, then $N T$ is $k$-semi-saturated. If $N B$ is a nested branch of $N T$ of length $n$ and the nesting rule can not be applied to the leaf of any branch $B$ at index $i \leq \min (n, k)$ in $N B$, then $N B$ is k-terminated. If every nested branch of NT of length $n$ is $\min (n, k)$-terminated, then $N T$ is $k$-terminated. If $N B$ is a nested branch of $N T$ that is $k$-terminated for each $k$, then $N B$ is terminated. If $N T$ is $k$-terminated for each $k$, then $N T$ is terminated.

We define the $k^{\prime}$-remainder of a branch, which is a refinement of the condition of that tableau, that is used by the subsequent definition of the set of extracted symbolic models.

Definition 28 ( $k^{\prime}$-Remainder of a Branch). Given a tableau $T$ for a condition $c$ over $C$, a monomorphism $q: C \hookrightarrow G$, a branch $B$ of $T$, and a prefix $P$ of $B$ of length $k^{\prime}>0$. If $R$ contains (a) each condition contained in $P$ unless it has been used in $P$ by the lift rule (being $\exists\left(m, c^{\prime}\right)$ or $\ell$ in the lift rule in Def. 20) and (b) the clauses of $c$ not used by the extension rule in $P$ (being $\vee\left(c_{1}, \ldots, c_{n}\right)$ in the extension rule in Def. 20), then $\langle C, \wedge R\rangle$ is the $k^{\prime}$-remainder of $B$.

The set of symbolic models extracted from a nested branch $N B$ is a set of certain $k^{\prime}$-remainders of branches of $N B$. In the example given in Figure 13 we extracted three symbolic models from the four nested branches of the nested tableau.

Definition 29 (Symbolic Model Extracted from a Nested Branch). If $N T$ is a nested tableau for a condition $c$ over $C$, $N B$ is a $k$-terminated and $k$-semi-saturated nested branch of $N T$ of length $n \leq k, B$ is the branch at index $n$ of length $k^{\prime}$ in $N B, B$ is open, $B$ contains no positive literals, then the $k^{\prime}$-remainder of $B$ is the symbolic model extracted from $N B$. The set of all such extracted symbolic models from $k$ terminated and $k$-semi-saturated nested branches of $N T$ is denoted $\mathcal{S}_{N T, k}$

Based on the previously introduced definitions of soundness, completeness, and minimal representability of sets of symbolic models w.r.t. graph properties we are now ready to verify the corresponding results on the algorithm $\mathcal{A}$.

Theorem 2 (Soundness). If NT is a nested tableau for a graph property $p$, then $\mathcal{S}_{N T, k}$ is sound w.r.t. $p$.

Theorem 3 (Completeness). If $N T$ is a finite terminated nested tableau for a graph property $p$, $k$ is the maximal length of a nested branch in NT, then $\mathcal{S}_{N T, k}$ is complete w.r.t. $p$.

The algorithm $\mathcal{A}$ does not always terminate as can be seen from the example in Figure 10e. However, the symbolic models extracted at any point during the breadth-first construction of the (possibly infinite) nested tableau NT are a gradually extended underapproximation of the set of symbolic models $\langle C, c\rangle$ with finite graphs $C$ that can extracted from $N T$. Moreover, during such a breadth-first construction the set of openers $\exists\left(m: G_{1} \hookrightarrow G_{2}, c\right)$ of the branches that end nonterminated nested branches constitutes an overapproximation. This overapproximation encodes a lower bound on missing symbolic models in the sense that each symbolic model that may be discovered by further tableau construction (and each graph satisfying the graph property that is not covered by some symbolic model extracted already) contains some $G_{2}$ as a subgraph. 


$\begin{array}{ccc}\text { Generation } & \text { Compaction } & \text { Disambiguation } \\ \text { of Set of Symbolic Models } & \text { of Set of Symbolic Models } & \text { of Set of Symbolic Models } \\ \text { subsection } 7.3 & \text { subsection } 7.4 & \text { subsection } 7.5\end{array}$

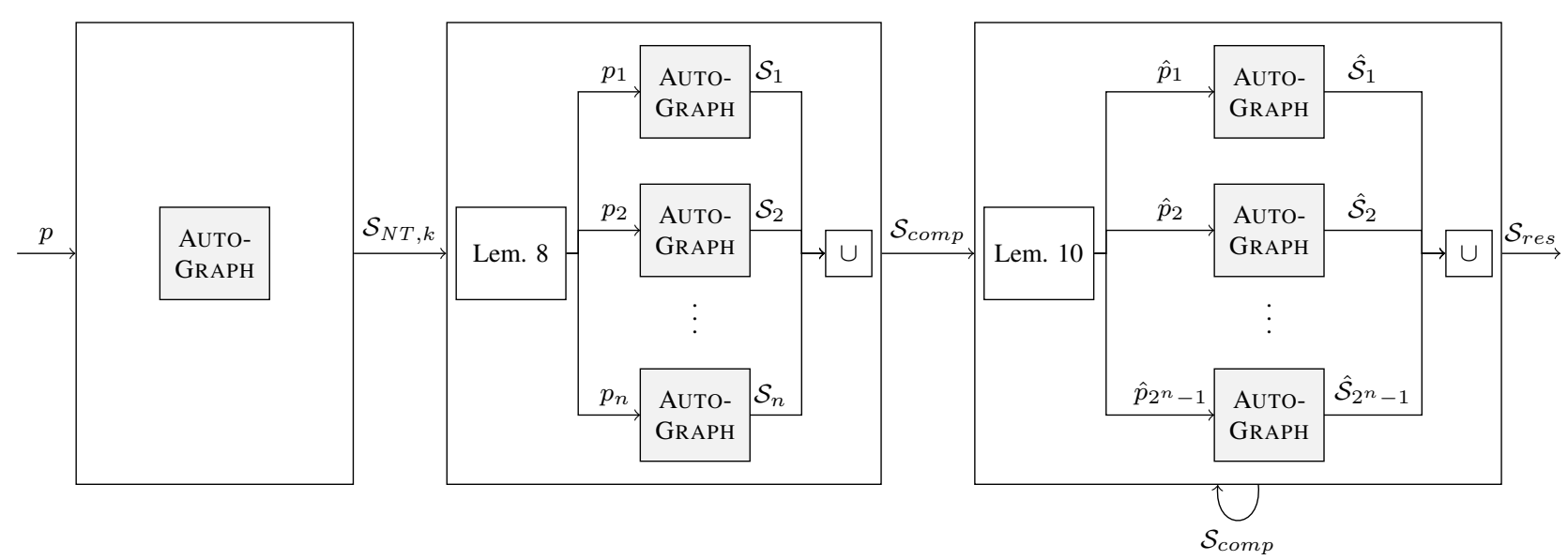

Figure 14: The symbolic model generation algorithm $\mathcal{A}$ with optional components for compaction and disambiguation. In each component the tool AUTOGRAPH is applied to $1, n$, and $2^{n}-1$ graph properties, respectively. Symbolic model generation obtains the set $\mathcal{S}_{N T, k}$ of symbolic models from a nested tableau $N T$. Then, compaction (using Lem. 8) constructs $n$ graph properties for the $n$ symbolic models in $\mathcal{S}_{N T, k}$ and removes symbolic models not contributing to the covered graphs resulting in $\mathcal{S}_{\text {comp }}$. Finally, disambiguation (using Lem. 10) constructs $2^{n}-1$ graph properties for the $n$ symbolic models in $\mathcal{S}_{\text {comp }}$ in each of its iterations, which disambiguate $\mathcal{S}_{\text {comp }}$ until no further disambiguation is necessary resulting in $\mathcal{S}_{\text {res }}$.

Furthermore, the extracted symbolic models $\langle C, c\rangle$ are most-explicit in the sense of minimal representability because the conditions $c$ contained in them define additional negative conditions that are satisfied by $C$ already. Of course, the graph $C$ may still have a set of attribute constraints that is satisfiable by various variable substitutions and, therefore, SMT solvers such as Z3 may be employed to derive examples of these variable substitutions resulting in grounded graphs (see Def. 13) to obtain most-explicit graphs that have a unique meaning w.r.t. the attributes as well.

Theorem 4 (Minimal Representability). If NT is a nested tableau for a graph property $p$, then $\mathcal{S}_{N T, k}$ is minimally representable w.r.t. $p$.

In the next subsection we explain how to modify sets of symbolic models extracted so far to additionally enforce compactness and nonambiguity.

\subsection{Compaction of Sets of Symbolic Models}

The finite set of symbolic models $\mathcal{S}_{N T, k}$ as obtained in the previous section is modified in this second step as follows to enforce compactness. This second step is intended to simplify $\mathcal{S}_{N T, k}$ and, hence, it may be aborted at any point, which may be necessary ocasionally because compaction (and disambiguation as well) are resource intensive and possibly nonterminating.

The following lemma supports the compaction of a set of symbolic models $\mathcal{S}$ into some restriction $\mathcal{S}^{\prime}$ of it by test- ing an emptiness condition. This emptiness condition can be expressed by refutability of a graph property.

Lemma 8 (Compaction). A subset $\mathcal{S}^{\prime}$ of the set $\mathcal{S}$ covers the same graphs as $\mathcal{S}$ iff covered $\left(\mathcal{S}-\mathcal{S}^{\prime}\right)-\operatorname{covered}\left(\mathcal{S}^{\prime}\right)=\emptyset$ iff $\vee\left\{\exists\left(i_{C}, c\right) \mid\langle C, c\rangle \in \mathcal{S}-\mathcal{S}^{\prime}\right\} \wedge \neg \vee\left\{\exists\left(i_{C}, c\right) \mid\langle C, c\rangle \in \mathcal{S}^{\prime}\right\}$ is refutable.

We apply this lemma by testing for each symbolic model $s$ in $\mathcal{S}_{N T, k}$ whether it can be removed from $\mathcal{S}_{N T, k}$ without altering the set of covered graphs. This iteration over the symbolic models may not terminate because the tableau procedure is only refutationally complete, i.e., AUTOGRAPH is only guaranteed to terminate on unsatisfiable graph properties. The resulting set $\mathcal{S}_{\text {comp }}$ of symbolic models is compact as desired.

Theorem 5 (Compactness). If NT is a nested tableau for a graph property $p$, then $\mathcal{S}_{\text {comp }} \subseteq \mathcal{S}_{N T, k}$ is compact.

Note, in [46] a weaker form of compactness has been enforced, which may be called binary compactness because it considers only two symbolic models at once.

As a special case we consider sets of symbolic models where the conditions contained in the symbolic models are equivalent to true. While such sets of symbolic models (with at least two elements) are ambiguous (e.g., the union of both minimal models proves the ambiguity) we can enforce compactness as follows.

Lemma 9 (Compactness for Symbolic Models with Trivial Remainder). The set of symbolic models $\mathcal{S}=\left\{\left\langle C_{i}, \wedge \emptyset\right\rangle \mid\right.$ 
$i \in I\}$ is compact iff for all two distinct symbolic models $\left\langle C_{1}, \wedge \emptyset\right\rangle$ and $\left\langle C_{2}, \wedge \emptyset\right\rangle$ contained in $\mathcal{S}$ there is no monomorphism $m: C_{1} \hookrightarrow C_{2}$.

As expected compactification using Lem. 9 is usually much faster than compaction using Lem. 8. However, even in this simple case we are required to find monomorphisms, which amounts to the NP-complete subgraph isomorphism problem. Nonetheless, since the handled graphs are typed and small (by construction we generate minimal models by operating only on the graphs from the conditions rather than operating on instance graphs) the required time for finding the monomorphisms is usually not problematic.

Usually the symbolic model generation procedure does not generate symbolic models with trivial remainder as required by Lem. 9. However, this lemma can be applied anyway in application scenarios where only the minimal models and not the remainders are of interest. Hence, replacing the remainders of the symbolic models obtained from AUTOGRAPH by $\wedge \emptyset$ implements, from this perspective, the selection of these minimal models and the above lemma then allows their compaction more efficiently than with Lem. 8 (before the replacement). The resulting compact set of symbolic models is then to be understood only as an enumeration of minimal models of the graph property from which the set $\mathcal{S}_{N T, k}$ has been generated.

\subsection{Disambiguation of Sets of Symbolic Models}

Subsequently we enforce nonambiguity of the ultimately obtained set $\mathcal{S}_{\text {res }}$ of symbolic models.

As a first step, we claim that a set of symbolic models is compact whenever it is nonambiguous showing that enforcing compactness can be skipped when nonambiguity is to be enforced (see Table 1 again for examples on the relationship between nonambiguity and compactness).

Corollary 1 (Nonambiguity implies Compactness). If $N T$ is a nested tableau for a graph property $p$ and $\mathcal{S} \subseteq \mathcal{S}_{N T, k}$, then $\mathcal{S}$ is compact it is nonambiguous.

The following lemma demonstates how a set of symbolic models $\mathcal{S}$ is disambiguated by considering all combinations of symbolic models in $\mathcal{S}$. For each such combination, which is given by a paritioning $\left(\mathcal{S}-\mathcal{S}^{\prime}, \mathcal{S}^{\prime}\right)$ for $\mathcal{S}^{\prime} \subsetneq \mathcal{S}$, we compute the symbolic models describing the graphs covered by $\mathcal{S}-\mathcal{S}^{\prime}$ and not covered by $\mathcal{S}^{\prime}$. The difference in this lemma can be expressed similarly as in Lem. 8.

Lemma 10 (Disambiguation). Let $\mathcal{S}$ be some given set of symbolic models. Then, the set covered $(\mathcal{S})$ is equal to the set $\bigcup\left\{\bigcap\left\{\operatorname{covered}(s) \mid s \in \mathcal{S}-\mathcal{S}^{\prime}\right\}-\operatorname{covered}\left(\mathcal{S}^{\prime}\right) \mid \mathcal{S}^{\prime} \subsetneq \mathcal{S}\right\}$.

However, for computational complexity we can observe that the number of cases, given by the subsets $\mathcal{S}^{\prime}$, is exponential in the size of $\mathcal{S}$. Furthermore, for each paritioning we obtain a condition that is a conjunctions of positive and negative literals and, hence, we apply AUTOGRAPH to each of these conditions to obtain for each set $\mathcal{S}^{\prime}$ a set of equivalent symbolic models. While the set of symbolic models generated by one of these graph properties may be ambiguous, the sets generated for the different sets of symbolic models $\mathcal{S}^{\prime}$ are nonambiguous.

For disambiguation we recursively apply Lem. 10 to split generated symbolic models enforcing nonambiguity of the set $\mathcal{S}_{\text {res }}$ obtained upon termination.

As for the the generation of symbolic models explained in the previous subsection and the compaction procedure explained above we may also abort the disambiguation procedure prematurely (e.g., once the designated resources are used up) still obtaining a meaningful result.

Currently we are unable to prevent noncompactness or ambiguity of the set of symbolic models generated by algorithm $\mathcal{A}$ on the fly (for example, by preventing some kinds of symmetries) during the computation without a similar impact on runtime.

\subsection{Exploration of Sets of Symbolic Models}

We believe that the exploration of further graphs satisfying a given property $p$ based on the symbolic models is often desireable. In fact, covered $\left(\mathcal{S}_{\text {res }}\right)$ can be explored according to Def. 24 by selecting $\langle C, c\rangle \in \mathcal{S}_{\text {res }}$, by generating a mono $m: C \hookrightarrow G$ to a new finite candidate graph $G$, and by deciding $m \models c$. Then, an entire automatic exploration can proceed by selecting the symbolic models $\langle C, c\rangle \in \mathcal{S}_{\text {res }}$ in a round-robin manner using an enumeration of the monos leaving $C$ in each case. However, the exploration may also be guided interactively restricting the considered symbolic models and monos.

For example, consider Figure 13 where a set of two symbolic models is obtained by application of algorithm $\mathcal{A}$ to the graph property $p^{13}$. In an interactive exploration we may want to decide whether the graph $\square \square \odot$ also satisfies $p^{13}$. In fact, since there is a monomorphism $m: \square \square \hookrightarrow \square \square \triangleright$ from the minimal model of $s_{1}$ to the graph to be tested that satisfies the remainder of $s_{1}$ we derive $\square \square \curvearrowright \models p^{13}$. However, the choice of the symbolic model is also in this case relevant because any morphism $m: \square \hookrightarrow \square \square \gg$ from the minimal model of $s_{3}$ to the graph to be tested does not satisfy the remainder of $s_{3}$ thereby not allowing the derivation of $\square \square \curvearrowright \models p^{13}$.

An entire enumeration is often not feasible, since many properties (e.g., true) have infinitely many models. However, we believe that it may prove useful in many application scenarios to obtain a finitely representable guidance to construct every possible finite model if needed. The set of symbolic models represents such a guidance indeed.

As mentioned above we will take advantage of explorability more explicitly in the future. In particular, it could be adapted to generate large sets of graphs or large, realistic graphs, for example, in the context of performance testing.

Moreover, in the context of coverage-based testing, the minimal models that we derive directly from our symbolic models are not necessarily already realistic enough to the user. This is true in particular when using attribute constraints 
as in the class diagram in Figure 2 because SMT solvers such as Z3 are not designed to return satisfying models for attribute constraints that take the intended meaning of the variables such as first name or spoken languages into account. The user might want to enlarge the models (possibly interactively) and determine whether this enlargement is consistent with the specification. However, we believe that the minimal models of a condition, which we are able to generate, are most likely already reasonable test input sets.

\section{Implementation}

In this section we introduce AUTOGRAPH by focussing in subsection 8.1 on the external characteristics and limitations of AUTOGRAPH (deferring a discussion on the features until section 9 where we apply AUTOGRAPH to examples and measure the performance) and in subsection 8.2 on the implementation details of the tableau construction procedure from algorithm $\mathcal{A}$ presented in section 6 .

\subsection{Functional Properties of AUtoGrapH}

We implemented the algorithm $\mathcal{A}$ platform-independently using JAVA as our new tool AUTOGRAPH. The inputs and outputs of AUTOGRAPH (i.e., attributed graph properties, the contained attributed graph morphisms with their attributed graphs, the used attributed constraints with their algebraic specifications, the used type graphs, and the generated sets of symbolic models) are XML files satisfying a custom XSD schema [52]. We support the different use cases from Figure 1 as follows: for an invalid query we return an empty set of symbolic models, for a valid query we return either only the first symbolic model generated or generate (if possible) the entire set of symbolic models (optionally executing compaction or disambiguation).

For the attributes and attribute constraints AUTOGRAPH uses Z3 via its JAVA bindings and has built-in support for the specification in Def. 30 to allow for attributes and attribute constraints over booleans, integers, and strings. Using custom algebraic specifications implementing complex functional programs is problematic for the automated reasoning of AUTOGRAPH in general because Z3 will fail to decide satisfiability when attribute constraints are too complex. Many SMT solvers such as Z3 have, besides deciding satisfiability, also support for generating some (or a sequence) of models for satisfied properties. While AUTOGRAPH does not compute certain grounded graphs, using this feature this may be of interest in various application domains. Finally, AUTOGRAPH uses Z3 to simplify attribute constraints and, hence, to keep them small. This is helpful because attribute constraints are growing in the algorithm due to the operation shift where, intuitively, the union of two sets of attribute constraints is computed (actually, the variables occurring in the sets of attribute constraints of the graphs $C_{1}$ and $C_{2}$ in Def. 17 are renamed according to $m_{1}^{\prime}$ and $m_{2}^{\prime}$ before computing the union of the two resulting sets).
When converting a graph condition into CNF in Step 3 (see Def. 19) we need to check whether the set of attribute constraints of the contained graphs are satisfiable. However, the SMT solver may time-out without returning a definite answer to such a satisfiability problem (as opposed to the oracle assumed in section 6 and section 7) depending on the attribute constraints. In this case we assume satisfiability by default, which may result in the generation of symbolic models without grounded graphs (a scenario that does not occur in section 7 due to the assumption of an oracle) and, in addition, it may be the reason for a nonterminating computation of AUTOGRAPH in cases where the known unsatisfiability would have prevented further execution by removal of the considered literal (see Step 3 in Def. 19). Alternatively, we could have assumed that sets of attribute constraints are unsatisfiable when the SMT solvers does not deliver a definite result. The premature abortion of the tableau based symbolic model generation procedure would imply that refutational completeness is no longer satisfied as not all symbolic models are generated.

For the computational complexity of the symbolic model generation algorithm we may notice that some elementary constructions used (such as conversion to CNF using [·], existence and enumeration of monomorphisms of a given type, and pair factorization as used in shift) have exponential worst case execution time. However, as explained at the end of section 5 , the operation [.] has typically no noticable impact and the problems of deciding existence and of enumeration of monomorphisms of a given type as well as pair factorization are applied during the execution of our algorithm, by design, only on minimal models instead of arbitrary instance graphs. Hence, we believe, also based on our tool-based evaluation in section 9 , that in many practical applications the runtime will be acceptable.

For decreased overall execution times AUTOGRAPH supports the usage of multithreading for various of its building blocks: in particular for the three high-level operations of tableau based symbolic model generation (which is considered in more detail below), compaction, and disambiguation. For the symbolic model generation we consider all open nested branches in parallel, and for compaction and disambiguation we check the satisfiability of the constructed graph properties in parallel using AUTOGRAPH.

\subsection{Implementation Details of AUTOGRAPH}

For limiting the memory consumption during the symbolic model generation, we discard the parts of the nested tableau that are not required for subsequent computations as follows. The implemented algorithm uses a queue (used to enforce the breadth-first construction) of configurations where every configuration represents the last branch of a nested branch of the nested tableau currently constructed (the parts of the nested tableau not given by these branches are thereby not represented in memory). The algorithm starts with a single initial configuration, applies one construction rule (see Figure 15) 


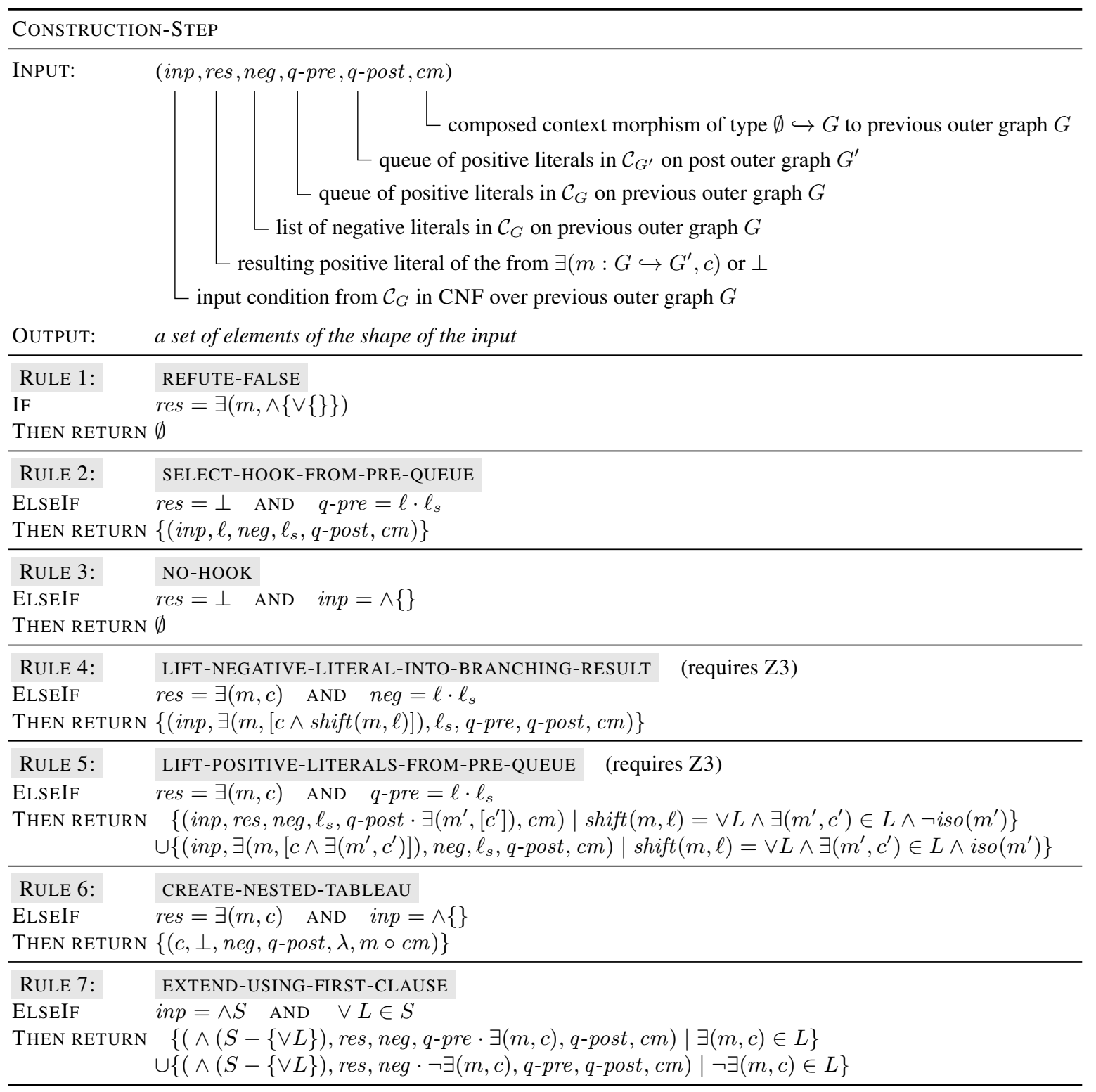

Figure 15: The construction-step that is implemented as a part of AUTOGRAPH and that is used iteratively by AUTOGRAPH to generate symbolic models where $\ell$ is a literal, $\ell_{s}$ is a list of literals, and $L$ is a set of literals. RULE 1 stops further generation if the current result res is unsatisfiable by having a subcondition that is equivalent to false. RULE 2 ensures that hooks are selected from the queue q-pre (if it is not empty) where fairness of hook selection is enforced by priorizing and ordering the positive literals that are successors of positive literals not chosen as hooks before. RULE 3 if the queue $q$-pre can not be used to select a hook and no clause remains, the nested branch is terminated and a symbolic model can be extracted by taking $\langle G, \wedge n e g\rangle$ where $G$ is the codomain of $\mathrm{cm}$. RULE 4 implements the lifting rule (see Def. 20) for negative literals taken from neg. RULE 5 implements the lifting rule (see Def. 20) for positive literals taken from $q$-pre; if the morphism of the resulting positive literal is an isomorphism, as forbidden for literals in CNF, we move an equivalent condition in CNF into the current hook (also implementing the lift rule) instead of moving the literal to the queue $q$-post because the conversion of the positive literal into CNF may not result in a conjunction of positive literals that could be added to the queue q-post. RULE 6 implements the nesting rule (see Def. 22). RULE 7 implements the extension rule (see Def. 20) constructing for each literal of a certain clause a new configuration to represent the different nested branches. 
inserting all results of that rule application to the queue, and terminates once the queue of configurations is empty.

The configurations contain the information that is necessary to continue the further construction of the nested tableau (also ensuring fair selection of hooks) and to extract the symbolic models whenever one is obtained.

The configurations are tuples of the form (inp, res, neg, $q$-pre, $q$-post, $\mathrm{cm}$ ) where inp is a condition $c$ over $C$ in CNF (the construction of the tableau starts with an initially provided condition in CNF from which clauses are removed one after another resulting in the remaining input condition inp), res is $\perp$ when no hook has been selected or a positive literal $\exists\left(m: C \hookrightarrow D, c^{\prime}\right)$ into which the other literals from the branch are lifted, neg is a list of negative literals over $C$ from clauses already handled (this list is emptied as soon as a positive literal has been chosen for res), q-pre is a queue of positive literals over $C$ from which the first element is chosen for the res component, q-post is a queue of positive literals: once res is a chosen positive literal $\exists\left(m: C \hookrightarrow D, c^{\prime}\right)$ we shift the elements from $q$-pre over $m$ to obtain elements of $q$-post, and $\mathrm{cm}$ is the composition of the morphisms from the openers of the nested branch constructed so far and is used to eventually obtain symbolic models (if they exist).

The implemented algorithm is started with the queue containing, for a graph property $p$, the unique initial configuration $\left([p], \perp, \lambda, \lambda, \lambda, i d_{\emptyset}\right)$ where $\lambda$ denotes the empty list.

The construction rules return for each single configurations a finite set of such configurations and are checked in the order given where only the first applicable rule is used. The construction rules are explained for better readability directly in Figure 15.

For soundness of the implemented algorithm based on the construction rules, reconsider Def. 28 where the set $R$ used in the condition $\wedge R$ recovers the desired information similarly to how it is captured in the configurations. The separation into different elements in the configurations then allows for queue handling and determinization.

\section{Evaluation}

In this section we analyze the four graph database queries, which were formalized as graph properties in Figure 3, Figure 4 , Figure 5, and Figure 6 , by checking their validity and by generating symbolic models for them by application of AUTOGRAPH. See Figure 1 again for a visualization of the general workflow. Note, the algorithm $\mathcal{A}$ implemented in AUTOGRAPH performs the refutability check, the satisfiability check, and the model generation at once. Hence, the set of symbolic models generated by AUTOGRAPH is sufficient (if AUTOGRAPH terminates) to answer the three given questions of whether a graph query is valid, invalid, and how graph databases look like when the graph query can be matched.

As a first step of our evaluation we have applied AUTOGRAPH to the four graph properties and all binary combinations of them measuring the number of symbolic models gen-

\begin{tabular}{c|r|r|r}
\hline Graph Property & \multicolumn{3}{|c}{ Symbolic Model Generation } \\
\cline { 2 - 4 } & number & 1 Thread & 4 Threads \\
\hline$p^{3}$ & 3 & $7 \mathrm{~ms}$ & $5 \mathrm{~ms}$ \\
$p^{4}$ & 1 & $3 \mathrm{~ms}$ & $1 \mathrm{~ms}$ \\
$p^{5}$ & 2 & $165 \mathrm{~ms}$ & $90 \mathrm{~ms}$ \\
$p^{6}$ & 1 & $104 \mathrm{~ms}$ & $103 \mathrm{~ms}$ \\
$p^{3} \wedge p^{4}$ & 96 & $1089 \mathrm{~ms}$ & $789 \mathrm{~ms}$ \\
$p^{3} \wedge p^{5}$ & 136 & $9802 \mathrm{~ms}$ & $6596 \mathrm{~ms}$ \\
$p^{3} \wedge p^{6}$ & 68 & $7270 \mathrm{~ms}$ & $5231 \mathrm{~ms}$ \\
$p^{4} \wedge p^{5}$ & 294 & $17365 \mathrm{~ms}$ & $13439 \mathrm{~ms}$ \\
$p^{4} \wedge p^{6}$ & 147 & $24769 \mathrm{~ms}$ & $14652 \mathrm{~ms}$ \\
$p^{5} \wedge p^{6}$ & 378 & $97043 \mathrm{~ms}$ & $50290 \mathrm{~ms}$ \\
$p_{3 v} \wedge p_{w f}$ & 99 & $99 \mathrm{~ms}$ & $99 \mathrm{~ms}$ \\
\hline
\end{tabular}

Table 2: Analysis results for the four graph database queries formalized in Figure 3, Figure 4, Figure 5, and Figure 6. The durations in the last two columns is the average over five runs. The specification of the used machine is as follows $256 \mathrm{~GB}$ DDR4, $2 \times$ E5-2643 Xeon @ 3.4 GHz $\times 6$ cores $\times 2$ threads.

erated as well as the duration of the generation where compaction and disambiguation have not been performed.

From the results presented in Table 2 we can draw the conclusion that all four queries are valid queries, i.e., for each of the four queries at least one graph database exists that matches the query. Also, for the binary combinations we derive that the queries do not exclude each other, that is, there are for each case graph databases that simultaneously match both queries. The four graph properties do not use a deep nested structure, which results in a narrow nested tableau in the beginning of the computation. This leads to the situations that some threads have not available leaf to work on in the beginning. Still, we can already observe a reasonable speed up when using multiple threads for the given graph properties.

As a second step we can inspect the symbolic models generated. They are depicted in Figure 16 where their remainders have been omitted for readability.

\section{Conclusion and Outlook}

We presented an automated reasoning approach for attributed graph properties. It includes both a refutationally complete tableau based reasoning method and a symbolic model generation procedure. The attributed graph properties are equivalent to FOL on graphs for the graph part. Our algorithms assume the existence of an oracle for solving attribute constraints in the properties. It allows for flexible adoption of different available SMT solvers in the actual implementation. Attribute reasoning is neatly separated from graph reasoning by a dedicated logic for attributed graph properties separating both parts.

Our refutation procedure and symbolic model generation algorithm are highly integrated. Since the latter is designed to compute a complete overview of all possible models, it 


\begin{tabular}{|c|c|c|}
\hline$P:$ Person & -1 10 & $P_{\text {res }}:$ Person \\
\hline $\mathrm{id}=\arg _{1}$ & . KHIOW & $\begin{array}{l}\text { id }=x_{\text {res }} \\
\text { firstName }=\arg _{2}\end{array}$ \\
\hline
\end{tabular}

\begin{tabular}{|c|l|l|l|l|}
\hline$P:$ Person & $:$ knows & $:$ Person & $:$ knows & \multicolumn{1}{c|}{$P_{\text {res }}:$ Person } \\
\cline { 1 - 1 } id $=\arg _{1}$ & & $\begin{array}{l}\text { id }=x_{r e s} \\
\text { firstName }=\arg _{2}\end{array}$ \\
\hline
\end{tabular}

\begin{tabular}{|c|c|c|c|c|c|c|}
\hline$P:$ Person & 1 & : Person & 1 & :Person & & $P_{\text {res }}:$ Person \\
\hline $\mathrm{id}=\arg _{1}$ & Ws & &. $\mathrm{K}$ & & KIIOW: & $\begin{array}{l}\text { id }=x_{\text {res }} \\
\text { firstName }=\arg _{2}\end{array}$ \\
\hline
\end{tabular}

(a) The three minimal models generated for graph property $p^{3}$ from Figure 3.

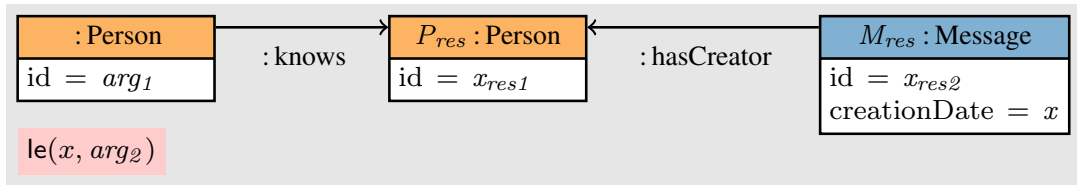

(b) The unique minimal models generated for graph property $p^{4}$ from Figure 4.

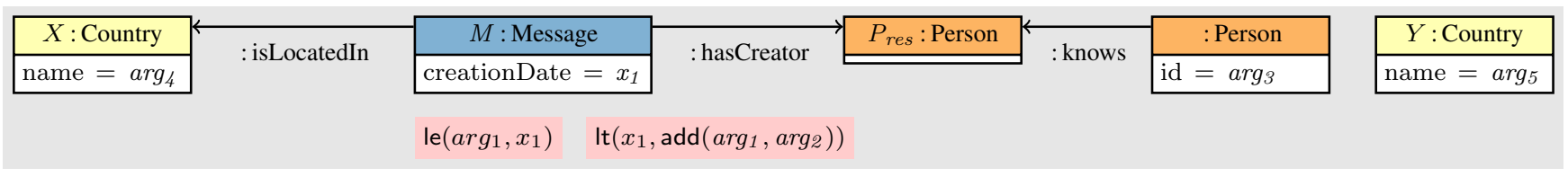

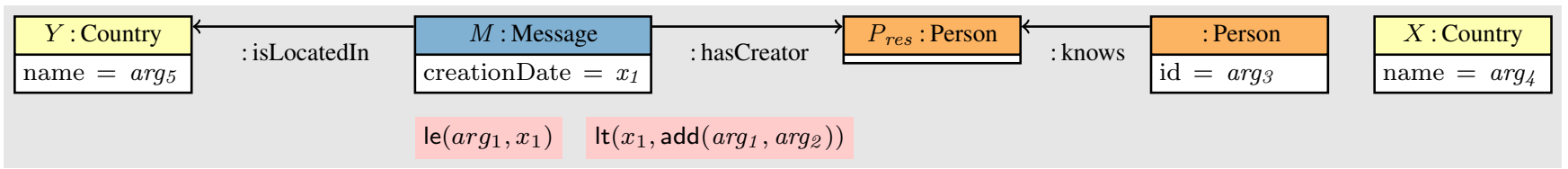

(c) The two minimal models generated for graph property $p^{5}$ from Figure 5.

\begin{tabular}{|c|c|c|c|c|c|c|}
\hline$T_{\text {res }}:$ Tag & \multirow{2}{*}{ : hasTag } & : Post & \multirow{2}{*}{ : hasCreator } & $P_{1}:$ Person & \multirow{2}{*}{ : knows } & :Person \\
\hline $\mathrm{id}=x_{r e s}$ & & creationDate $=x_{1}$ & & & & $\mathrm{id}=\arg _{1}$ \\
\hline $\operatorname{le}\left(\arg _{2}, x_{1}\right)$ & \multicolumn{6}{|c|}{$\operatorname{It}\left(x_{1}, \operatorname{add}\left(\arg _{2}, \arg _{3}\right)\right)$} \\
\hline
\end{tabular}

(d) The unique minimal model generated for graph property $p^{6}$ from Figure 6.

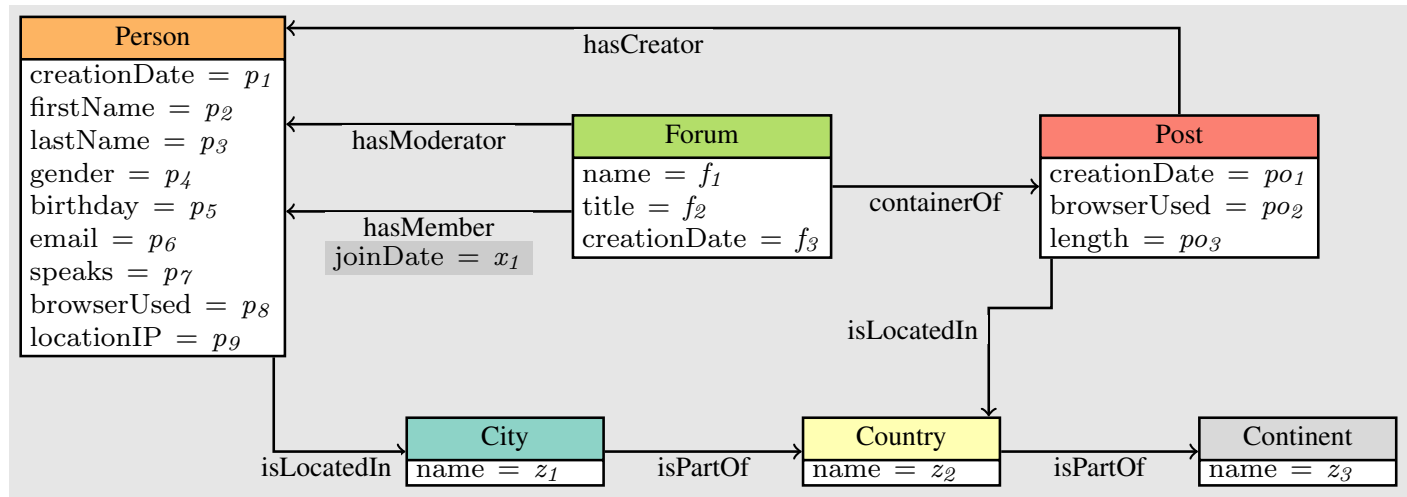

(e) A minimal model generated by AUTOGRAPH when requiring at least one vertex of type Forum and the satisfaction of all multiplicity constraints stated in the class diagram given in Figure 2. These multiplicity constraints have been formalized by graph properties along the lines of Figure 10c and Figure 10d.

Figure 16: Minimal models generated by AUTOGRAPH for the graph properties from Figure 3, Figure 4, Figure 5, and Figure 6. 
is at the same time able to refute a property if the overview turns out to be empty. Our symbolic model generation algorithm is innovative in the sense that it is designed to generate a finite set of symbolic models that is sound, complete (upon termination), compact, nonambiguous, minimally representable, and flexibly explorable. Moreover, the algorithm is parallelizable because every employed thread can work on one leaf of the nested tableau to be constructed. The approach is implemented in our tool, called AUTOGRAPH.

As future work we will attempt to determine descriptions of subsets of graph properties for which termination of AUTOGRAPH is guaranteed. Moreover, we aim at applying, evaluating, and optimizing our approach further w.r.t. other application scenarios such as test generation for the graph database domain [7], but also to other domains such as model-driven engineering, where our approach can be used, e.g., to generate test models for model transformations $[5,19,30]$. We also aim at generalizing our approach to more expressive graph properties able to encode, e.g., path-related properties [41, $40,29]$. We moreover aim at supporting graph properties to state temporal properties on graphs where nodes and edges are equipped with attributes specifying their lifespan. Finally, the work on exploration of extracted symbolic models as well as reducing their number during tableau construction is an ongoing task. In particular, we are working on algorithms for the generation of a subset of the complete set of symbolic models that is suitably diverse. These extensions are valuable when the complete set of symbolic models is too large or its generation requires too many resources.

\section{References}

1. Serge Abiteboul, Richard Hull, and Victor Vianu, editors. Foundations of Databases: The Logical Level. Addison-Wesley Longman Publishing Co., Inc., Boston, MA, USA, 1st edition, 1995.

2. Renzo Angles, Marcelo Arenas, Pablo Barceló, Aidan Hogan, Juan L. Reutter, and Domagoj Vrgoc. Foundations of modern graph query languages. CoRR, abs/1610.06264, 2016.

3. Renzo Angles and Claudio Gutierrez. Survey of Graph Database Models. ACM Comput. Surv., 40(1):1:1-1:39, February 2008 .

4. Kacper Bak, Zinovy Diskin, Michal Antkiewicz, Krzysztof Czarnecki, and Andrzej Wasowski. Clafer: unifying class and feature modeling. Software and System Modeling, 15(3):811845, 2016.

5. Benoit Baudry. Testing model transformations: A case for test generation from input domain models. In Model Driven Engineering for Distributed Real-time Embedded Systems. Hermes, 2009.

6. Thomas Beyhl, Dominique Blouin, Holger Giese, and Leen Lambers. On the operationalization of graph queries with generalized discrimination networks. In Echahed and Minas [12], pages $170-186$.

7. Raquel Blanco and Javier Tuya. A test model for graph database applications: an mda-based approach. In Tanja E. J. Vos, Sigrid Eldh, and Wishnu Prasetya, editors, Proceedings of the 6th International Workshop on Automating Test Case Design, Selec- tion and Evaluation, A-TEST 2015, Bergamo, Italy, August 3031, 2015, pages 8-15. ACM, 2015.

8. E. F. Codd. A relational model of data for large shared data banks. Commun. ACM, 13(6):377-387, June 1970.

9. Bruno Courcelle. The expression of graph properties and graph transformations in monadic second-order logic. In Rozenberg [44], pages 313-400.

10. Gwendal Daniel, Gerson Sunyé, and Jordi Cabot. Umltographdb: Mapping conceptual schemas to graph databases. In Isabelle Comyn-Wattiau, Katsumi Tanaka, Il-Yeol Song, Shuichiro Yamamoto, and Motoshi Saeki, editors, Conceptual Modeling - 35th International Conference, volume 9974 of Lecture Notes in Computer Science, pages 430-444, 2016.

11. Frederik Deckwerth. Static Verification Techniques for Attributed Graph Transformations. PhD thesis, Darmstadt University of Technology, Germany, 2017.

12. Rachid Echahed and Mark Minas, editors. Graph Transformation - 9th International Conference, ICGT 2016, in Memory of Hartmut Ehrig, Held as Part of STAF 2016, Vienna, Austria, July 5-6, 2016, Proceedings, volume 9761 of Lecture Notes in Computer Science. Springer, 2016.

13. Hartmut Ehrig, Karsten Ehrig, Ulrike Prange, and Gabriele Taentzer. Fundamentals of algebraic graph transformation. Springer-Verlag, 2006.

14. Hartmut Ehrig, Ulrike Golas, Annegret Habel, Leen Lambers, and Fernando Orejas. $\mathcal{M}$-adhesive transformation systems with nested application conditions. part 1: parallelism, concurrency and amalgamation. Mathematical Structures in Computer Science, 24(4), 2014.

15. Hartmut Ehrig, Reiko Heckel, Grzegorz Rozenberg, and Gabriele Taentzer, editors. Graph Transformations, 4th International Conference, ICGT 2008, Leicester, United Kingdom, September 7-13, 2008. Proceedings, volume 5214 of Lecture Notes in Computer Science. Springer, 2008.

16. Hartmut Ehrig and Bernd Mahr. Fundamentals of Algebraic Specification 1: Equations und Initial Semantics, volume 6 of EATCS Monographs on Theoretical Computer Science. Springer, 1985.

17. Holger Giese and Barbara König, editors. Graph Transformation - 7th International Conference, ICGT 2014, Held as Part of STAF 2014, York, UK, July 22-24, 2014. Proceedings, volume 8571 of Lecture Notes in Computer Science. Springer, 2014.

18. Martin Gogolla and Frank Hilken. Model validation and verification options in a contemporary UML and OCL analysis tool. In Andreas Oberweis and Ralf H. Reussner, editors, Modellierung 2016, 2.-4. März 2016, Karlsruhe, volume 254 of LNI, pages 205-220. GI, 2016.

19. Carlos A. González and Jordi Cabot. Test data generation for model transformations combining partition and constraint analysis. In Davide Di Ruscio and Dániel Varró, editors, Theory and Practice of Model Transformations - 7th International Conference, ICMT 2014, Held as Part of STAF 2014, York, UK, July 21-22, 2014. Proceedings, volume 8568 of Lecture Notes in Computer Science, pages 25-41. Springer, 2014.

20. Annegret Habel, Reiko Heckel, and Gabriele Taentzer. Graph grammars with negative application conditions. Fundam. Inform., 26(3/4):287-313, 1996.

21. Annegret Habel and Karl-Heinz Pennemann. Correctness of high-level transformation systems relative to nested conditions. Mathematical Structures in Computer Science, 19(2):245-296, 2009. 
22. Reiner Hähnle. Tableaux and related methods. In John Alan Robinson and Andrei Voronkov, editors, Handbook of Automated Reasoning (in 2 volumes), pages 100-178. Elsevier and MIT Press, 2001.

23. Reiko Heckel and Annika Wagner. Ensuring consistency of conditional graph rewriting - a constructive approach. Electr. Notes Theor. Comput. Sci., 2:118-126, 1995.

24. Ethan K. Jackson, Tihamer Levendovszky, and Daniel Balasubramanian. Reasoning about metamodeling with formal specifications and automatic proofs. In Jon Whittle, Tony Clark, and Thomas Kühne, editors, Model Driven Engineering Languages and Systems, 14th International Conference, MODELS 2011, Wellington, New Zealand, October 16-21, 2011. Proceedings, volume 6981 of Lecture Notes in Computer Science, pages 653-667. Springer, 2011.

25. Ethan K. Jackson and Janos Sztipanovits. Constructive techniques for meta- and model-level reasoning. In Gregor Engels, Bill Opdyke, Douglas C. Schmidt, and Frank Weil, editors, Model Driven Engineering Languages and Systems, 10th International Conference, MoDELS 2007, Nashville, USA, September 30 - October 5, 2007, Proceedings, volume 4735 of Lecture Notes in Computer Science, pages 405-419. Springer, 2007.

26. Christian Krause, Daniel Johannsen, Radwan Deeb, Kai-Uwe Sattler, David Knacker, and Anton Niadzelka. An sql-based query language and engine for graph pattern matching. In Echahed and Minas [12], pages 153-169.

27. Leen Lambers and Fernando Orejas. Tableau-based reasoning for graph properties. In Giese and König [17], pages 17-32.

28. Microsoft Corporation. Z3. https://github.com/ Z3Prover/z 3. Accessed: 2017-09-19.

29. Aleksandar Milicevic, Joseph P. Near, Eunsuk Kang, and Daniel Jackson. Alloy*: A general-purpose higher-order relational constraint solver. In Antonia Bertolino, Gerardo Canfora, and Sebastian G. Elbaum, editors, 37th IEEE/ACM International Conference on Software Engineering, ICSE 2015, Florence, Italy, May 16-24, 2015, Volume 1, pages 609-619. IEEE Computer Society, 2015.

30. Alix Mougenot, Alexis Darrasse, Xavier Blanc, and Michèle Soria. Uniform random generation of huge metamodel instances. In Richard F. Paige, Alan Hartman, and Arend Rensink, editors, Model Driven Architecture - Foundations and Applications, 5th European Conference, ECMDA-FA 2009, Enschede, The Netherlands, June 23-26, 2009. Proceedings, volume 5562 of Lecture Notes in Computer Science, pages 130 145. Springer, 2009.

31. Tim Nelson, Salman Saghafi, Daniel J. Dougherty, Kathi Fisler, and Shriram Krishnamurthi. Aluminum: principled scenario exploration through minimality. In David Notkin, Betty H. C. Cheng, and Klaus Pohl, editors, 35th International Conference on Software Engineering, ICSE '13, San Francisco, CA, USA, May 18-26, 2013, pages 232-241. IEEE Computer Society, 2013.

32. Fernando Orejas. Attributed graph constraints. In Ehrig et al. [15], pages 274-288.

33. Fernando Orejas, Hartmut Ehrig, and Ulrike Prange. A logic of graph constraints. In José Luiz Fiadeiro and Paola Inverardi, editors, Fundamental Approaches to Software Engineering, 11th International Conference, FASE 2008, Held as Part of the Joint European Conferences on Theory and Practice of Software, ETAPS 2008, Budapest, Hungary, March 29-April 6, 2008. Proceedings, volume 4961 of Lecture Notes in Computer Science, pages 179-198. Springer, 2008.
34. Fernando Orejas, Hartmut Ehrig, and Ulrike Prange. Reasoning with graph constraints. Formal Asp. Comput., 22(3-4):385-422, 2010.

35. Fernando Orejas and Leen Lambers. Symbolic attributed graphs for attributed graph transformation. ECEASST, 30, 2010.

36. Fernando Orejas and Leen Lambers. Lazy graph transformation. Fundam. Inform., 118(1-2):65-96, 2012.

37. Karl-Heinz Pennemann. An algorithm for approximating the satisfiability problem of high-level conditions. Electr. Notes Theor. Comput. Sci., 213(1):75-94, 2008.

38. Karl-Heinz Pennemann. Resolution-like theorem proving for high-level conditions. In Ehrig et al. [15], pages 289-304.

39. Karl-Heinz Pennemann. Development of Correct Graph Transformation Systems, PhD Thesis. Dept. Informatik, Univ. Oldenburg, 2009.

40. Christopher M. Poskitt and Detlef Plump. Verifying monadic second-order properties of graph programs. In Giese and König [17], pages 33-48.

41. Hendrik Radke. Hr* graph conditions between counting monadic second-order and second-order graph formulas. ECEASST, 61, 2013.

42. Hendrik Radke, Thorsten Arendt, Jan Steffen Becker, Annegret Habel, and Gabriele Taentzer. Translating essential OCL invariants to nested graph constraints focusing on set operations. In Francesco Parisi-Presicce and Bernhard Westfechtel, editors, Graph Transformation - 8th International Conference, ICGT 2015, Held as Part of STAF 2015, L'Aquila, Italy, July 21-23, 2015. Proceedings, volume 9151 of Lecture Notes in Computer Science, pages 155-170. Springer, 2015.

43. Arend Rensink. Representing first-order logic using graphs. In Hartmut Ehrig, Gregor Engels, Francesco Parisi-Presicce, and Grzegorz Rozenberg, editors, Graph Transformations, Second International Conference, ICGT 2004, Rome, Italy, September 28 - October 2, 2004, Proceedings, volume 3256 of Lecture Notes in Computer Science, pages 319-335. Springer, 2004.

44. Grzegorz Rozenberg, editor. Handbook of Graph Grammars and Computing by Graph Transformations, Volume 1: Foundations. World Scientific, 1997.

45. Rick Salay and Marsha Chechik. A generalized formal framework for partial modeling. In Alexander Egyed and Ina Schaefer, editors, Fundamental Approaches to Software Engineering - 18th International Conference, FASE 2015, Held as Part of the European Joint Conferences on Theory and Practice of Software, ETAPS 2015, London, UK, April 11-18, 2015. Proceedings, volume 9033 of Lecture Notes in Computer Science, pages 133-148. Springer, 2015.

46. Sven Schneider, Leen Lambers, and Fernando Orejas. Symbolic model generation for graph properties. In Marieke Huisman and Julia Rubin, editors, Fundamental Approaches to Software Engineering - 20th International Conference, FASE 2017, Held as Part of the European Joint Conferences on Theory and Practice of Software, ETAPS 2017, Uppsala, Sweden, April 22-29, 2017, Proceedings, volume 10202 of Lecture Notes in Computer Science, pages 226-243. Springer, 2017.

47. Sven Schneider, Leen Lambers, and Fernando Orejas. Symbolic Model Generation for Graph Properties (Extended Version). Number 115 in Technische Berichte des Hasso-PlattnerInstituts für Softwaresystemtechnik an der Universität Potsdam. Universitätsverlag Potsdam, Hasso Plattner Institute (Germany, Potsdam), 1 edition, 22017.

48. Nicole Schweikardt, Thomas Schwentick, and Luc Segoufin. Algorithms and theory of computation handbook. chapter 
Database Theory: Query Languages, pages 19, 1-34. Chapman \& Hall/CRC, 2010.

49. Oszkár Semeráth and Dániel Varró. Graph constraint evaluation over partial models by constraint rewriting. In Esther Guerra and Mark van den Brand, editors, Theory and Practice of Model Transformation - 10th International Conference, ICMT 2017, Held as Part of STAF 2017, Marburg, Germany, July 17-18, 2017, Proceedings, volume 10374 of Lecture Notes in Computer Science, pages 138-154. Springer, 2017.

50. Oszkár Semeráth, András Vörös, and Dániel Varró. Iterative and incremental model generation by logic solvers. In Perdita Stevens and Andrzej Wasowski, editors, Fundamental Approaches to Software Engineering - 19th International Conference, FASE 2016, Held as Part of the European Joint Conferences on Theory and Practice of Software, ETAPS 2016, Eindhoven, The Netherlands, April 2-8, 2016, Proceedings, volume 9633 of Lecture Notes in Computer Science, pages 87-103. Springer, 2016.

51. The Linked Data Benchmark Council (LDBC). Social network benchmark. https://github.com/ldbc/ldbc_snb_ docs. Accessed: 2017-08-21.

52. The World Wide Web Consortium (W3C). W3c xml schema definition language (xsd) 1.1 part 1: Structures, 2012.

53. Peter T. Wood. Query languages for graph databases. SIGMOD Record, 41(1):50-60, 2012.

\section{A Some Details on Autograph}

Definition 30 (Z3 Signature).

sorts: bool, int, string

opns: true : $\rightarrow$ bool

false : $\rightarrow$ bool

not : bool $\rightarrow$ bool

and $:$ bool bool $\rightarrow$ bool

or : bool bool $\rightarrow$ bool

xor : bool bool $\rightarrow$ bool

implies : bool bool $\rightarrow$ bool

eq $_{\text {bool }}$ : bool bool $\rightarrow$ bool

ifthenelse $_{\text {bool }}:$ bool bool bool $\rightarrow$ bool

zero : $\rightarrow$ int

succ : int $\rightarrow$ int

pred $:$ int $\rightarrow$ int

minus : int $\rightarrow$ int

add : int int $\rightarrow$ int

sub : int int $\rightarrow$ int

mul : int int $\rightarrow$ int

mod : int int $\rightarrow$ int

rem : int int $\rightarrow$ int

power : int int $\rightarrow$ int

eq $_{\text {int }}$ : int int $\rightarrow$ bool

gt : int int $\rightarrow$ bool

It : int int $\rightarrow$ bool

ge : int int $\rightarrow$ bool

le : int int $\rightarrow$ bool

ifthenelse $_{\text {int }}$ : bool int int $\rightarrow$ int

empty : $\rightarrow$ string

concat : string string $\rightarrow$ string

length : string $\rightarrow$ int

contains : string string $\rightarrow$ bool

indexOf : string string int $\rightarrow$ int

replace : string string string $\rightarrow$ string

prefixOf : string string $\rightarrow$ bool

suffixOf : string string $\rightarrow$ bool

at : string int $\rightarrow$ string

extract : string int int $\rightarrow$ string

eq string $_{\text {: string string } \rightarrow \text { bool }}$

ifthenelse $_{\text {string }}$ : bool string string $\rightarrow$ string

Furthermore, we assume sufficient operations for constructing values of string as terms such as $a, \ldots, z, 0, \ldots, 9,-$, SPACE : $\rightarrow$ string. 


\section{B Categorical Preliminaries and Properties of GRAPHSSTA}

Lemma 11 (GRAPHSSTA: Characterization of the Monomorphisms, Epimorphisms, and Isomorphisms). A graph morphism $f: G \rightarrow G^{\prime}$ from the category GRAPHSSTA is a mono(morphism) (epi(morphism)) (iso(morphism)), if each of its components is injective (surjective) (bijective), respectively. And, for isomorphisms we additionally require that the reversed implication from Def. 11 holds as well, i.e., for all $\sigma \in \mathcal{V}_{\Sigma_{A_{2}}, \Sigma_{A_{2}}}: \sigma \models f_{A X}\left(\Phi_{1}\right)$ implies $\sigma \models \Phi_{2}$.

Proof (idea). Due to the componentwise characterization.

Definition 31 ( $\mathcal{E}$ - $\mathcal{M}$-Factorization). Given a category, a set $\mathcal{E}$ of epimorphisms, and a set $\mathcal{M}$ of monomorphisms. The category has $\mathcal{E}$-M-Factorizations, if

- (existence) for each $f: A \rightarrow C$ there are $(e: A \rightarrow K) \in$ $\mathcal{E}$ and $(m: K \hookrightarrow C) \in \mathcal{M}$ s.t. $m \circ e=f$ and

- (uniqueness) for $\left(e^{\prime}: A \rightarrow K^{\prime}\right) \in \mathcal{E}$ and $\left(m^{\prime}: K^{\prime} \hookrightarrow\right.$ $C) \in \mathcal{M}$ with $m^{\prime} \circ e^{\prime}=f$ there is $i: K \hookrightarrow K^{\prime}$ with $i \circ e=e^{\prime}$ and $m^{\prime} \circ i=m$.

Definition 32 (Jointly Epimorphic Morphisms [13, Definition A.16, p. 334]). Two morphisms $e_{1}: A_{1} \rightarrow B$ and $e_{2}: A_{2} \rightarrow B$ of a category are Jointly Epimorphic, if any two morphisms $g, h: B \rightarrow C$ are equal whenever $g \circ e_{i}=h \circ e_{i}$ (for each $1 \leq i \leq 2$ ).

Pair Factorization (cf. [13, Definition 5.25, p. 122]) has the intuition that any two morphisms $f_{1}$ and $f_{2}$ with common codomain $C$ coincide (in the sense of mapping to the same elements) on a well-defined subgraph $K$ of $C$. That $K$ does not include further elements (on which the two morphisms do not coincide) is expressed by stating that the morphisms $e_{1}$ and $e_{2}$ are jointly epimorphic and the coincidence is expressed by stating that $m$ is a monomorphism together with the commutation.

Definition 33 ( $\mathcal{E}^{\prime}$ - $\mathcal{M}$-Pair Factorization). For a given category, a set $\mathcal{E}^{\prime}$ of pairs $\left(f_{1}, f_{2}\right)$ of jointly epi morphisms, and a set $\mathcal{M}$ of monomorphisms. The category has $\mathcal{E}^{\prime}-\mathcal{M}$-Pair Factorizations, if for each two morphisms $f_{1}: A_{1} \rightarrow C$ and $f_{2}: A_{2} \rightarrow C$ there are $\left(e_{1}: A_{1} \rightarrow K, e_{2}: A_{2} \rightarrow K\right) \in \mathcal{E}^{\prime}$ and $(m: K \hookrightarrow C) \in \mathcal{M}$ s.t. $m \circ e_{i}=f_{i}$ (for each $1 \leq i \leq 2$ ).

Definition 34 (Binary Coproduct). A category has binary coproducts, if for every $A_{i}$ (with $1 \leq i \leq 2$ ) there are $f_{i}$ : $A_{i} \rightarrow C$ (for each $1 \leq i \leq 2$ ) s.t. (the following universal property holds) for all $g_{i}: A_{i} \rightarrow X$ there is $h: C \rightarrow X$ with $h \circ f_{i}=g_{i}($ for each $1 \leq i \leq 2)$.

\section{Lemma 12 (GraPhSSTA has Binary Coproducts).}

Proof (idea). The binary coproduct $C$ with morphisms $f_{1}$ and $f_{2}$ from Def. 34 is constructed componentwise using the disjoint union, as usual.
For the category GRAPHSSTA we use as $\mathcal{E}$ the set of all epimorphisms, as $\mathcal{M}$ the set of all monomorphisms, and as $\mathcal{E}^{\prime}$ the set of all pairs of jointly epimorphic morphisms.

\section{Lemma 13 (GRAPHSSTA has $\mathcal{E}$ - $\mathcal{M}$-Factorization).}

Proof (idea). The morphisms $e$ and $m$, required according to Def. 31, are constructed componentwise for a morphism $f$.

\section{Lemma 14 (GRAPHSSTA has $\mathcal{E}^{\prime}-\mathcal{M}$-Pair Factorization).}

Proof (idea). Analogously to [13, Remark 5.26, p. 122] we construct the $\mathcal{E}^{\prime}-\mathcal{M}$-Pair Factorizations using $\mathcal{E}$ - $\mathcal{M}$-Factorizations (based on Lem. 13) and binary coproducts (based on Lem. 12).

\section{Proofs}

Proof (of Lem. 2). Part1 (ㄷ).

$$
\begin{aligned}
& G \in \llbracket \exists\left(i_{C}, \vee S\right) \rrbracket \\
& \Longrightarrow i_{G}=\exists\left(i_{C}, \vee S\right)
\end{aligned}
$$

for some $q: C \hookrightarrow G$

$$
\Longrightarrow q \models \vee S
$$

for some $c \in S$

$$
\begin{aligned}
& \Longrightarrow q \models c \\
& \Longrightarrow q \circ i_{C} \models \exists\left(i_{C}, c\right) \\
& \Longrightarrow i_{G} \models \exists\left(i_{C}, c\right) \\
& \Longrightarrow G \in \llbracket \exists\left(i_{C}, c\right) \rrbracket \\
& \Longrightarrow G \in \bigcup\left\{\llbracket \exists\left(i_{C}, c\right) \rrbracket \mid c \in S\right\}
\end{aligned}
$$

Part2 (つ).

$$
G \in \bigcup\left\{\llbracket \exists\left(i_{C}, c\right) \rrbracket \mid c \in S\right\}
$$

for some $c \in S$

$$
\begin{aligned}
& \Longrightarrow G \in \llbracket \exists\left(i_{C}, c\right) \rrbracket \\
& \Longrightarrow i_{G} \models \exists\left(i_{C}, c\right)
\end{aligned}
$$

for some $q: C \hookrightarrow G$

$$
\begin{aligned}
& \Longrightarrow q \models c \\
& \Longrightarrow q \models \vee S \\
& \Longrightarrow q \circ i_{C} \models \exists\left(i_{C}, \vee S\right) \\
& \Longrightarrow i_{G} \models \exists\left(i_{C}, \vee S\right) \\
& \Longrightarrow G \in \llbracket \exists\left(i_{C}, \vee S\right) \rrbracket
\end{aligned}
$$

Proof (of Lem. 5). Part1 (ㄷ).

$$
\begin{array}{r}
G \in \llbracket \exists\left(i_{C_{1}}, \exists\left(m: C_{1} \hookrightarrow C_{2}, c\right)\right) \rrbracket \\
\Longrightarrow i_{G} \models \exists\left(i_{C_{1}}, \exists\left(m: C_{1} \hookrightarrow C_{2}, c\right)\right)
\end{array}
$$


for some $q_{1}: C_{1} \hookrightarrow G$

$$
\Longrightarrow q_{1} \vDash \exists\left(m: C_{1} \hookrightarrow C_{2}, c\right)
$$

for some $q_{2}: C_{2} \hookrightarrow G$

$$
\begin{aligned}
& \Longrightarrow q_{2}=c \text { and } q_{1}=q_{2} \circ m \\
& \Longrightarrow q_{2} \circ i_{C_{2}}=\exists\left(i_{C_{2}}, c\right) \\
& \Longrightarrow i_{G}=\exists\left(i_{C_{2}}, c\right) \\
& \Longrightarrow G \in \llbracket \exists\left(i_{C_{2}}, c\right) \rrbracket
\end{aligned}
$$

Part2 (つ).

$$
\begin{array}{r}
G \in \llbracket \exists\left(i_{C_{2}}, c\right) \rrbracket \\
\Longrightarrow i_{G}=\exists\left(i_{C_{2}}, c\right)
\end{array}
$$

for some $q_{2}: C_{2} \hookrightarrow G$

$$
\begin{aligned}
& \Longrightarrow q_{2} \models c \\
& \Longrightarrow q_{2} \circ m \models \exists\left(m: C_{1} \hookrightarrow C_{2}, c\right) \\
& \Longrightarrow q_{2} \circ m \circ i_{C_{1}} \models \exists\left(i_{C_{1}}, \exists\left(m: C_{1} \hookrightarrow C_{2}, c\right)\right) \\
& \Longrightarrow i_{G} \models \exists\left(i_{C_{1}}, \exists\left(m: C_{1} \hookrightarrow C_{2}, c\right)\right) \\
& \Longrightarrow G \in \llbracket \exists\left(i_{C_{1}}, \exists\left(m: C_{1} \hookrightarrow C_{2}, c\right)\right) \rrbracket
\end{aligned}
$$

Proof (of Lem. 6). Part1 ( $C$ is an element).

$$
\begin{aligned}
C \in \llbracket \exists\left(i_{C}, \wedge\left\{\neg \exists\left(m_{1}, c_{1}\right), \ldots, \neg \exists\left(m_{n}, c_{n}\right)\right\}\right) \rrbracket \\
\Longleftarrow i_{C} \models \exists\left(i_{C}, \wedge\left\{\neg \exists\left(m_{1}, c_{1}\right), \ldots, \neg \exists\left(m_{n}, c_{n}\right)\right\}\right)
\end{aligned}
$$

for $i d_{C}: C \hookrightarrow C$

$$
\Longleftarrow i d_{C} \models \wedge\left\{\neg \exists\left(m_{1}, c_{1}\right), \ldots, \neg \exists\left(m_{n}, c_{n}\right)\right\}
$$

for each $1 \leq i \leq n$ simultaneously

$$
\begin{aligned}
& \Longleftarrow i d_{C} \models \neg \exists\left(m_{i}, c_{i}\right) \\
& \Longleftarrow i d_{C} \not \models \exists\left(m_{i}, c_{i}\right)
\end{aligned}
$$

there is no $q_{i}: C_{i} \hookrightarrow G$ such that $q_{i}=c_{i}$ and $q_{i} \circ m_{i}=q$

$\Longleftarrow m_{i}$ is no isomorphism

Part2 (unique least element).

$$
C^{\prime} \in \llbracket \exists\left(i_{C}, \wedge\left\{\neg \exists\left(m_{1}, c_{1}\right), \ldots, \neg \exists\left(m_{n}, c_{n}\right)\right\}\right) \rrbracket
$$$$
\Longrightarrow i_{C^{\prime}} \models \llbracket \exists\left(i_{C}, \wedge\left\{\neg \exists\left(m_{1}, c_{1}\right), \ldots, \neg \exists\left(m_{n}, c_{n}\right)\right\}\right) \rrbracket
$$

for some $q: C \hookrightarrow C^{\prime}$

$$
\begin{aligned}
& \Longrightarrow q \models \wedge\left\{\neg \exists\left(m_{1}, c_{1}\right), \ldots, \neg \exists\left(m_{n}, c_{n}\right)\right\} \\
& \Longrightarrow C \subseteq C^{\prime}
\end{aligned}
$$

Proof (of Lem. 3). Part1 (ㄷ).

$$
\begin{gathered}
G \in \llbracket \exists\left(i_{C},(\wedge S) \wedge \exists\left(m: C \hookrightarrow C^{\prime}, c^{\prime}\right)\right) \rrbracket \\
\Longrightarrow i_{G}=\exists\left(i_{C},(\wedge S) \wedge \exists\left(m: C \hookrightarrow C^{\prime}, c^{\prime}\right)\right)
\end{gathered}
$$

for some $q_{1}: C \hookrightarrow G$

$$
\begin{aligned}
& \Longrightarrow q_{1} \models(\wedge S) \wedge \exists\left(m: C \hookrightarrow C^{\prime}, c^{\prime}\right) \\
& \Longrightarrow q_{1} \models \wedge S \text { and } q_{1} \models \exists\left(m: C \hookrightarrow C^{\prime}, c^{\prime}\right)
\end{aligned}
$$

for some $q_{2}: C^{\prime} \hookrightarrow G$

$$
\Longrightarrow q_{2}=c^{\prime} \text { and } q_{1}=q_{2} \circ \mathrm{m}
$$

also

$$
\begin{aligned}
& q_{1} \vDash \wedge S \\
\Longrightarrow & q_{2} \circ m \models \wedge S \\
\Longrightarrow & q_{2} \models \operatorname{shift}(m, \wedge S) \\
\Longrightarrow & q_{2} \models c^{\prime} \wedge \operatorname{shift}(m, \wedge S) \\
\Longrightarrow & q_{2} \circ m \models \exists\left(m, c^{\prime} \wedge \operatorname{shift}(m, \wedge S)\right) \\
\Longrightarrow & q_{2} \circ m \circ i_{C} \models \exists\left(i_{C}, \exists\left(m, c^{\prime} \wedge \operatorname{shift}(m, \wedge S)\right)\right) \\
\Longrightarrow & i_{G} \models \exists\left(i_{C}, \exists\left(m, c^{\prime} \wedge \operatorname{shift}(m, \wedge S)\right)\right) \\
\Longrightarrow & G \in \llbracket \exists\left(i_{C}, \exists\left(m, c^{\prime} \wedge \operatorname{shift}(m, \wedge S)\right)\right) \rrbracket
\end{aligned}
$$

Part2 ().

$$
\begin{array}{r}
G \in \llbracket \exists\left(i_{C}, \exists\left(m, c^{\prime} \wedge \operatorname{shift}(m, \wedge S)\right)\right) \rrbracket \\
\Longrightarrow i_{G}=\exists\left(i_{C}, \exists\left(m, c^{\prime} \wedge \operatorname{shift}(m, \wedge S)\right)\right)
\end{array}
$$

for some $q_{1}: C \hookrightarrow G$

$$
\Longrightarrow q_{1} \models \exists\left(m, c^{\prime} \wedge \operatorname{shift}(m, \wedge S)\right)
$$

for some $q_{2}: C^{\prime} \hookrightarrow G$

$$
\begin{aligned}
& \Longrightarrow q_{2} \models c^{\prime} \wedge \operatorname{shift}(m, \wedge S) \text { and } q_{1}=q_{2} \circ m \\
& \Longrightarrow q_{2} \models c^{\prime} \text { and } q_{2} \models \operatorname{shift}(m, \wedge S) \\
& \Longrightarrow q_{2} \circ m \models \exists\left(m, c^{\prime}\right)
\end{aligned}
$$

also

$$
\begin{aligned}
& \Longrightarrow q_{2}=\operatorname{shift}(m, \wedge S) \\
& \Longrightarrow q_{2} \circ m \models(\wedge S) \\
& \Longrightarrow q_{2} \circ m \models(\wedge S) \wedge \exists\left(m: C \hookrightarrow C^{\prime}, c^{\prime}\right) \\
& \Longrightarrow q_{2} \circ m \circ i_{C} \models \exists\left(i_{C},(\wedge S) \wedge \exists\left(m: C \hookrightarrow C^{\prime}, c^{\prime}\right)\right) \\
& \Longrightarrow i_{G} \models \exists\left(i_{C},(\wedge S) \wedge \exists\left(m: C \hookrightarrow C^{\prime}, c^{\prime}\right)\right) \\
& \Longrightarrow G \in \llbracket \exists\left(i_{C},(\wedge S) \wedge \exists\left(m: C \hookrightarrow C^{\prime}, c^{\prime}\right)\right) \rrbracket
\end{aligned}
$$

Proof (of Lem. 8). Part1.1 (if).

$$
\begin{aligned}
& \operatorname{covered}(\mathcal{S})=\operatorname{covered}\left(\mathcal{S}^{\prime}\right) \\
& \Longrightarrow \operatorname{covered}(\mathcal{S}) \subseteq \operatorname{covered}\left(\mathcal{S}^{\prime}\right)
\end{aligned}
$$

because $\operatorname{covered}\left(\mathcal{S}-\mathcal{S}^{\prime}\right) \subseteq \operatorname{covered}(\mathcal{S})$

$$
\begin{aligned}
& \Longrightarrow \operatorname{covered}\left(\mathcal{S}-\mathcal{S}^{\prime}\right) \subseteq \operatorname{covered}\left(\mathcal{S}^{\prime}\right) \\
& \Longrightarrow \operatorname{covered}\left(\mathcal{S}-\mathcal{S}^{\prime}\right)-\operatorname{covered}\left(\mathcal{S}^{\prime}\right)=\emptyset
\end{aligned}
$$

Part1.2 (only if).

$$
\begin{aligned}
& \operatorname{covered}\left(\mathcal{S}-\mathcal{S}^{\prime}\right)-\operatorname{covered}\left(\mathcal{S}^{\prime}\right)=\emptyset \\
& \Longrightarrow \operatorname{covered}\left(\mathcal{S}-\mathcal{S}^{\prime}\right) \subseteq \operatorname{covered}\left(\mathcal{S}^{\prime}\right)
\end{aligned}
$$

because covered $(\mathcal{S})-\operatorname{covered}\left(\mathcal{S}^{\prime}\right) \subseteq \operatorname{covered}\left(\mathcal{S}-\mathcal{S}^{\prime}\right)$

$\Longrightarrow \operatorname{covered}(\mathcal{S})-\operatorname{covered}\left(\mathcal{S}^{\prime}\right) \subseteq \operatorname{covered}\left(\mathcal{S}^{\prime}\right)$

$\Longrightarrow\left(\operatorname{covered}(\mathcal{S})-\operatorname{covered}\left(\mathcal{S}^{\prime}\right)\right)-\operatorname{covered}\left(\mathcal{S}^{\prime}\right)=\emptyset$

$\Longrightarrow \operatorname{covered}(\mathcal{S})-\operatorname{covered}\left(\mathcal{S}^{\prime}\right)=\emptyset$

$\Longrightarrow \operatorname{covered}(\mathcal{S}) \subseteq \operatorname{covered}\left(\mathcal{S}^{\prime}\right)$ 
because $\mathcal{S}^{\prime} \subseteq \mathcal{S}$ implies covered $\left(\mathcal{S}^{\prime}\right) \subseteq \operatorname{covered}(\mathcal{S})$

$$
\Longrightarrow \operatorname{covered}(\mathcal{S})=\operatorname{covered}\left(\mathcal{S}^{\prime}\right)
$$

Part2.

$$
\begin{aligned}
\vee & \left\{\exists\left(i_{C}, c\right) \mid\langle C, c\rangle \in \mathcal{S}-\mathcal{S}^{\prime}\right\} \\
& \wedge \neg \vee\left\{\exists\left(i_{C}, c\right) \mid\langle C, c\rangle \in \mathcal{S}^{\prime}\right\} \text { is refutable } \\
\Longleftrightarrow \llbracket \vee & \left\{\exists\left(i_{C}, c\right) \mid\langle C, c\rangle \in \mathcal{S}-\mathcal{S}^{\prime}\right\} \\
& \wedge \neg \vee\left\{\exists\left(i_{C}, c\right) \mid\langle C, c\rangle \in \mathcal{S}^{\prime}\right\} \rrbracket=\emptyset \\
\Longleftrightarrow \llbracket \vee & \left\{\exists\left(i_{C}, c\right) \mid\langle C, c\rangle \in \mathcal{S}-\mathcal{S}^{\prime}\right\} \rrbracket \\
& \cap \llbracket \neg \vee\left\{\exists\left(i_{C}, c\right) \mid\langle C, c\rangle \in \mathcal{S}^{\prime}\right\} \rrbracket=\emptyset \\
\Longleftrightarrow \operatorname{covered}\left(\mathcal{S}-\mathcal{S}^{\prime}\right) & \cap\left(\{G \mid G \text { is a graph }\}-\operatorname{covered}\left(\mathcal{S}^{\prime}\right)\right)=\emptyset \\
& \cap \operatorname{covered}\left(\mathcal{S}-\mathcal{S}^{\prime}\right)-\operatorname{covered}\left(\mathcal{S}^{\prime}\right)=\emptyset
\end{aligned}
$$

Proof (of Lem. 9).

- Part1 (if). Fix some $\langle C, \wedge \emptyset\rangle \in \mathcal{S}$.

Assume for the contradiction $\operatorname{covered}(\mathcal{S})=\operatorname{covered}(\mathcal{S}-$ $\{\langle C, \wedge \emptyset\rangle\})$.

Hence, for each $G \in \operatorname{covered}(\langle C, \wedge \emptyset\rangle)$ there is some other $\left\langle C^{\prime}, \wedge \emptyset\right\rangle \in \mathcal{S}$ s.t. $G \in \operatorname{covered}(\langle C, \wedge \emptyset\rangle)$.

Note that $C \in$ covered $\left(\left\langle C^{\prime}, \wedge \emptyset\right\rangle\right)$. Hence, we are able to pick some $\left\langle C^{\prime}, \wedge \emptyset\right\rangle$ s.t. $C \in \operatorname{covered}\left(\left\langle C^{\prime}, \wedge \emptyset\right\rangle\right)$.

Hence, there is a monomorphism $m: C^{\prime} \hookrightarrow C$.

This is a contradiction.

- Part2 (only if). Fix distinct $\langle C, \wedge \emptyset\rangle \in \mathcal{S}$ and $\left\langle C^{\prime}, \wedge \emptyset\right\rangle \in$ $\mathcal{S}$.

Assume for the contradiction that $m: C^{\prime} \hookrightarrow C$ is a monomorphism.

Hence covered $(\langle C, \wedge \emptyset\rangle) \subseteq \operatorname{covered}\left(\left\langle C^{\prime}, \wedge \emptyset\right\rangle\right)$.

Hence covered $(\mathcal{S})=\operatorname{covered}(\mathcal{S}-\{\langle C, \wedge \emptyset\rangle\})$.

Hence $\mathcal{S}$ is not compact.

This is a contradiction.

Proof (of Corollary 1). Let $\mathcal{S}$ be nonambiguous, (sound,) complete, minimally representable.

We show that $\mathcal{S}$ is compact.

Fix some $\langle C, c\rangle \in \mathcal{S}$.

We show that $\operatorname{covered}(\mathcal{S}) \neq \operatorname{covered}(\mathcal{S}-\{\langle C, c\rangle\})$.

- We show that $C \in \operatorname{covered}(\mathcal{S})$.

From minimally representability we have that $C \models p$.

From completeness we that $C \in \operatorname{covered}(\mathcal{S})$.

- We show that $C \notin \operatorname{covered}(\mathcal{S}-\{\langle C, c\rangle)$.

Assume for the contradiction that $\left\langle C^{\prime}, c^{\prime}\right\rangle \in \mathcal{S}-\{\langle C, c\rangle$ such that $C \in \operatorname{covered}\left(\left\langle C^{\prime}, c^{\prime}\right\rangle\right)$.

Then, covered $\left(\left\langle C^{\prime}, c^{\prime}\right\rangle\right) \cap$ covered $(\langle C, c\rangle) \neq \emptyset$ contradicts nonambiguity because $\left\langle C^{\prime}, c^{\prime}\right\rangle \neq\langle C, c\rangle$.

Lemma 15 (Satisfaction is a Congruence). Let $c_{1}$ and $c_{2}$ be conditions from $\mathcal{C}_{C}$ such that $\left\{q \mid q \models c_{1}\right\}=\{q \mid q \models$ $\left.c_{2}\right\}$. Then all following items are satisfied.

- $\left\{q|q| \wedge\left(S \cup\left\{c_{1}\right\}\right)\right\}=\left\{q|q| \wedge\left(S \cup\left\{c_{2}\right\}\right)\right\}$ for all finite $S \subseteq \mathcal{C}_{C}$.

$-\left\{q \mid q \models \neg c_{1}\right\}=\left\{q \mid q \models \neg c_{2}\right\}$.

$$
\begin{aligned}
& -\left\{q \mid q \models \exists\left(m: C^{\prime} \hookrightarrow C, c_{1}\right)\right\}=\left\{q | q | \exists \left(m: C^{\prime} \hookrightarrow\right.\right. \\
& \left.\left.C, c_{2}\right)\right\} \text { for every } m: C^{\prime} \hookrightarrow C .
\end{aligned}
$$

Proof (of Lem. 15). Fix $c_{1}, c_{2} \in \mathcal{C}_{C}$.

Assume (A) that $\left\{q \mid q \models c_{1}\right\}=\left\{q \mid q \models c_{2}\right\}$. In each case we show only one direction wlog.

- Fix some $S \subseteq \mathcal{C}_{C}$ that is finite.

Fix some $q: C \hookrightarrow G$ such that $q \models \wedge\left(S \cup\left\{c_{1}\right\}\right)$.

Hence, $q \models \wedge S$ and $q \models c_{1}$.

From (A) we have that $q \models c_{2}$.

Hence, $q \models \wedge\left(S \cup\left\{c_{2}\right\}\right)$.

- Fix some $q: C \hookrightarrow G$ such that $q \models \neg c_{1}$.

Hence, not $q \models c_{1}$.

From (A) we have that not $q \models c_{2}$.

Hence, $q \models \neg c_{2}$.

- Fix some $m: C^{\prime} \hookrightarrow C$.

Fix some $q^{\prime}: C^{\prime} \hookrightarrow G$ such that $q^{\prime} \models \exists\left(m: C^{\prime} \hookrightarrow\right.$ $\left.C, c_{1}\right)$.

Hence, there is some $q: C \hookrightarrow G$ such that $q \models c_{1}$ and $q \circ m=q^{\prime}$.

From (A) we have that $q \models c_{2}$.

Hence, $q^{\prime} \models \exists\left(m: C^{\prime} \hookrightarrow C, c_{2}\right)$.

Proof (of Lem. 4). The construction steps of [.] replace subterms.

By structural induction relying on Lem. 15 it is sufficient to consider how one condition $c_{1}$ is replaced by another condition $c_{2}$ over same graph in the sense of $\left\{q \mid q \models c_{1}\right\}=\{q \mid$ $\left.q \models c_{2}\right\}$.

We verify for all five steps of the operation [.] that the property is rephrased equivalently.

- Step 1: The unfolding of abbrevations is sound by default.

- Step 2: We assume that $\exists\left(i: A \hookrightarrow B, c_{1}\right)$ has been replaced by $c_{2}$.

We perform an induction on $c_{1}$.

- Case: $c_{1}=\neg c_{1}^{\prime}$ and, hence, $c_{2}=\exists\left(i: A \hookrightarrow B, \neg c_{2}^{\prime}\right)$ for some $c_{2}^{\prime}$

As induction hypothesis we assume that $\{q \mid q \models$ $\left.c_{1}^{\prime}\right\}=\left\{q \mid q \models c_{2}^{\prime}\right\}$. We have to show $\{q \mid q \models \exists(i$ : $\left.\left.A \hookrightarrow B, \neg c_{1}^{\prime}\right)\right\}=\left\{q|q| \exists\left(i: A \hookrightarrow B, \neg c_{2}^{\prime}\right)\right\}$, which is the direct consequence from Lem. 15 .

- Case: $c_{1}=\wedge\left\{c_{0,1}, \ldots, c_{n, 1}\right\}$ and, hence, $c_{2}=\wedge\left\{c_{0,2}\right.$, $\left.\ldots, c_{n, 2}\right\}$ for some $c_{0,2}, \ldots, c_{n, 2}$

As induction hypothesis we assume that $\{q|q|=$ $\left.c_{i, 1}\right\}=\left\{q|q| c_{i, 2}\right\}$ (for $0 \leq i \leq n$ ) We have to show $\left\{q|q|=\exists\left(i: A \hookrightarrow B, \wedge\left\{c_{0,1}, \ldots, c_{n, 1}\right\}\right)\right\}=$ $\left\{q \mid q \models \exists\left(i: A \hookrightarrow B, \wedge\left\{c_{0,2}, \ldots, c_{n, 2}\right\}\right)\right\}$, which is the direct consequence from Lem. 15.

- Case: $c_{1}=\exists\left(m: B \hookrightarrow B^{\prime}, c_{1}^{\prime}\right)$ and, hence, $c_{2}=$ $\exists\left(m \circ i, c_{1}^{\prime}\right)$

We have to show $\{q \mid q \models \exists(i: A \hookrightarrow B, \exists(m$ : $\left.\left.\left.B \hookrightarrow B^{\prime}, c_{1}^{\prime}\right)\right)\right\}=\left\{q \mid q \models \exists\left(m \circ i, c_{1}^{\prime}\right)\right\}$, which holds directly application of Def. 16 .

- Step 3: We show that $\{q \mid q \models \exists(m: A \hookrightarrow B, c)\}=\{q \mid$ $q \models \vee \emptyset\}$ if $\{q \mid q \models \exists(m: A \hookrightarrow B, c)\}$ is empty. This is trivially the case because $\{q \mid q \models \vee \emptyset\}$ is also empty. 
- Step 4: The mentioned replacement rules $\neg(\wedge S) \doteq \vee\{\neg c \mid c \in S\}$,

$\neg(\vee S) \doteq \wedge\{\neg c \mid c \in S\}$, and

$\neg \neg c \doteq c$

are obvisouly sound in the sense of:

$\{q \mid q \models l h s\}=\{q \mid q \models$ rhs $\}$.

- Step 5: The mentioned replacement rules

$\wedge\left(S \cup\left\{\wedge S^{\prime}\right\}\right) \doteq \wedge\left(S \cup S^{\prime}\right)$,

$\vee\left(S \cup\left\{\vee S^{\prime}\right\}\right) \doteq \vee\left(S \cup S^{\prime}\right)$,

$\wedge\left(S \cup\left\{\vee S^{\prime}\right\}\right) \doteq \vee\left\{\wedge(S \cup\{c\}) \mid c \in S^{\prime}\right\}$, and

$\vee\left(S \cup\left\{\wedge S^{\prime}\right\}\right) \doteq \wedge\left\{\vee(S \cup\{c\}) \mid c \in S^{\prime}\right\}$

are obvisouly sound in the sense of:

$\{q \mid q \models l h s\}=\{q \mid q \models$ rhs $\}$. 